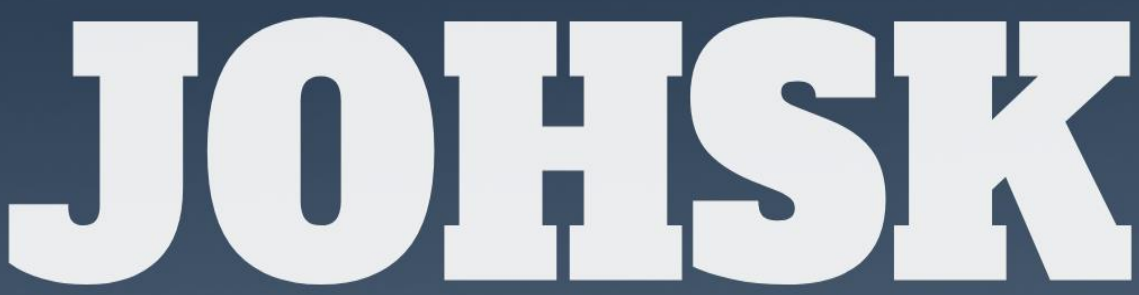

\title{
JOURNAL OF HEALTH, SPORTS, AND KINESIOLOGY
}

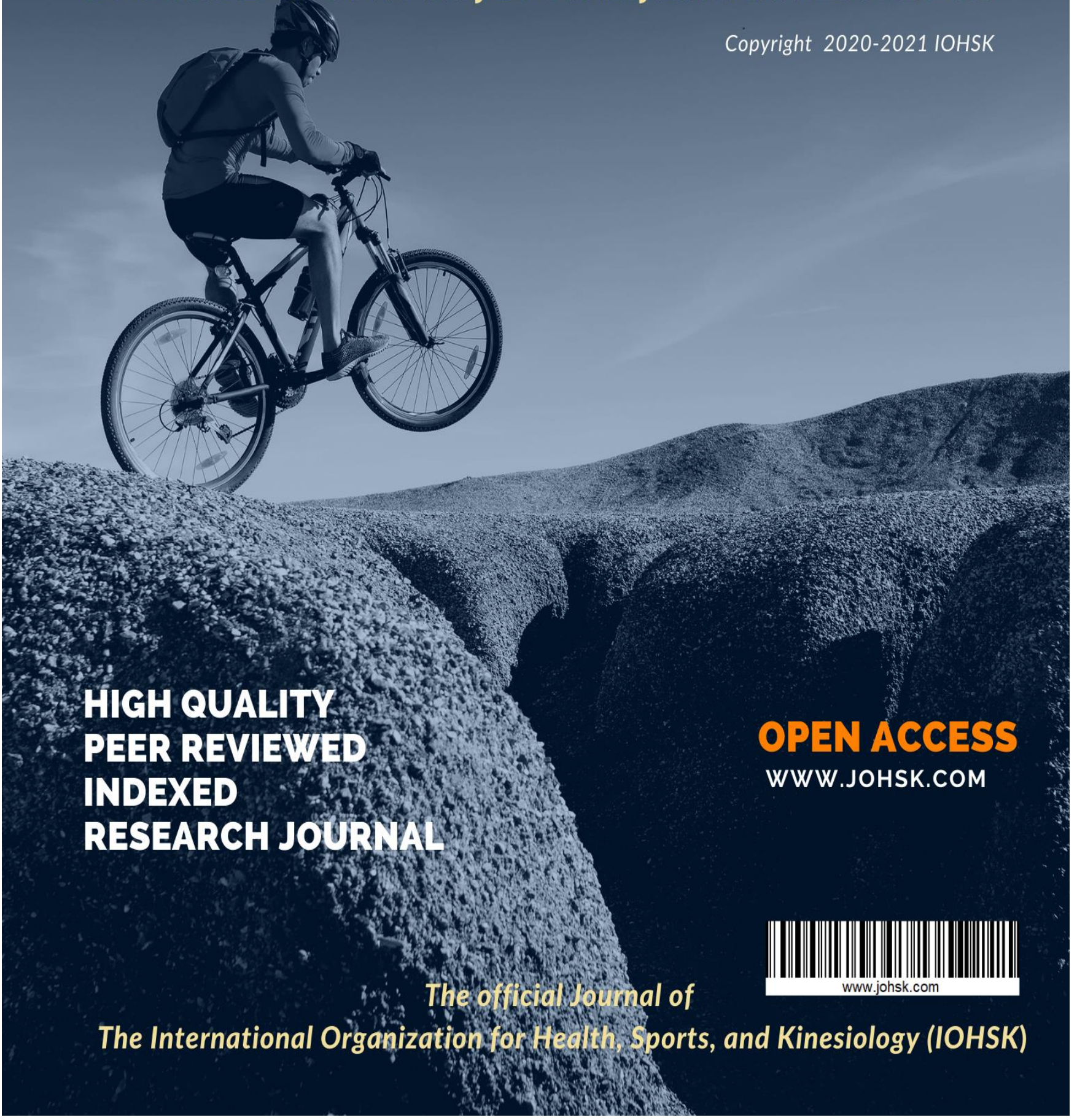




\section{JOURNAL OF HEALTH, SPORTS, \& KINESIOLOGY}

Copyright $($ C 2020-2021 by the International Organization for Health, Sports, and Kinesiology (IOHSK). www.iohsk.org. The Journal of Health, Sports, and Kinesiology (JOHSK) publishes research articles in the fields of Health, Sports, and Kinesiology. JOHSK is an open access and peer-reviewed research journal that is published by the International Organization for Health, Sports, and Kinesiology (IOHSK). The Journal of Health, Sports, and Kinesiology (JOHSK) publishes four times a year. The JOHSK provides a platform for the researchers, academicians, professionals, practitioners, and students to impart and share knowledge. JOHSK welcomes and acknowledges high-quality theoretical and empirical original research papers, case studies, review papers, literature reviews, book reviews, conceptual framework, analytical and simulation models, technical notes from researchers, academicians, professionals, practitioners, and students from all over the world. Work must be of a quality and context that would be of interest to an international readership. Manuscripts for special issues and individual papers can be on any contemporary health, sports, and kinesiology topics of international interest. Research findings of high-quality research involving any discipline and methodology related to Health, Sports, and Kinesiology will be welcome. However, the journal's aim and scope are to ensure it publishes high quality research that could potentially inform research. The research findings do not have to be comparative (in the sense of comparing aspects of Health, Sports, and Kinesiology in different countries or cultures): a manuscript may report research carried out in just one regional location or sociocultural setting. Work can be drawn from any context or research paradigm. However, the Journal tends to publish empirical research studies that have clear significance to an international readership.

Editor: Young Sub Kwon, USA

Publishing Editor: Hosung So, USA

Associate Editors:

Boungjin Kang, Elizabeth City State University, USA

Stephen Hankil Shin, Belmont University, USA

Soojin Yoo, University of Texas, Rio Grande Valley, USA
Send editorial correspondence to: Editor-in-Chief, Young Sub Kwon, Ph.D. Department of Kinesiology \& Recreation Administration, 1 Harpst Stree Humboldt State University Arcata, CA 95521, USA
Send business correspondence to: Publishing Editor Hosung So, Ph.D. Department of Kinesiology, HP 202 5500 University Parkway California State University, San Bernardino San Bernardino, CA 92407, USA

\section{Editorial Board Committee}

Junhyung Baek, Gyeongin National University of Education, Korea Chungil Chae, Kean University, Wenzhou, China Seung Ho Chang, San Jose State University, USA Soon Mi Choi, Midwestern State University, USA Sungchan Hong, University of Tsukuba, Japan Jiho Kim, Wingate University, USA Joon Young Kim, Syracuse University, USA Jun Kim, Southern Illinois University, USA Jun Kim, Southern Minhyun Kim, Sam Houston State University, USA Yeon Kim, California State University, San Bernardino, USA Youngje Kim, Chung-Ang University, Korea Ji Young Kim, Sangmyung University, Korea MinHyuk Kwon, California State Polytechnic University, USA Jihyun Lee, San Jose State University, USA Kyoung Eun Lee, Texas A\&M University, Corpus Christi, USA Soyoun Lim, Mississippi State University, USA Hasan Mavi, Eastern Illinois University, USA Issei Ogasawara, Osaka University, Japan Eung-Soo Oh, Donga University, Korea Hyun-Kyoung Oh, California State University, San Bernardino, USA Yoonsin Oh, University of Wisconsin, Eau Claire, USA Yoonsin Oh, University Of Wisconsin, Eau Claire, US Jinkyung Park, Georgia College and State University, USA Kyungjin Park, University of the Sacred Heart, Japan Kyungjin Park, University of the Sacred Heart, Japar
Meungguk Park, Southern Illinois University, USA Sungje Park, Chung-Ang University, Korea Sitha Phongphilbool, Chulalongkorn University, Thailand Seungmin Shin, King Fahd University of Petroleum \& Minerals, Saudi Arabia Pinar Yaprak, Gazi University, Turkey

Jae Yom, University of lllinois, Springfield, USA

\section{Publishing Editorial Committee}

aemin Ha, University of Northern Colorado, USA Boram Lim, University of Nevada, Las Vegas, USA Jongho Moon, University of South Carolina, USA Hyeonho YU, Arizona State University, USA

\section{Editorial Review Committee}

Daniel Balderson, University of Lethbridge, Canada Joy Barros, University of Texas, Rio Grande Valley, USA Elise Brown, Oakland University, USA

Cuauhtemoc Carboni, Cuyamaca College, USA Yu-Kai Chang, National Taiwan Normal University, Taiwan Kibum Cho, Hanyang University, Korea Jeongwon Choi, University of New Mexico, USA Germina Cosma, University of Craiova, Romania Christopher Gentry, California State University, San Bernardino, USA Yu-Lun Huang, University of Wisconsin, Eau Claire, USA John A. Johnson, Keimyung University, Korea Christian Guerrero Juarez, University of California, Irvine, USA Sombat Karnjanakit, Chulalongkorn University, Thailand Doyeop Kim, Troy University, USA MinJung Kim, University of Minnesota, USA Younglee Kim, California State University, San Bernardino, USA Jemin Kim, New Mexico State University, USA Garry Kuan, Universiti Sains Malaysia, Malaysia Don Lee, University of Houston, USA Heesu Lee, Koreg University, Kored Seonghun Lee, Flagler College, USA Hui Yin Ler, Tunku Abdul Rahman University College, Malaysia Julia Lee, Northwestern University Feinberg School of Medicine, USA Seungkil Lim, Dongshin University, Korea Kisuk Min, University of Texas, El Paso, USA Kisuk Min, University of Texas, El Paso, USA Emilio M. A. Millan, Autonomous University of Baja Californa,
Shannon Mulhearn, University of Nebraska, Kearney, USA Junichi Nishida, Kindai University, Japan Taewoong Oh, Yongin University, Korea Wagner Prado, California State University, San Bernardino, USA Ukje Sung, Columbia University, USA Sung-Sang Yoo, Seoul National University, Korea

JOSHK is a high-quality open access and peer-reviewed research journal. Work must be undertaken in an ethical manner. Research must have been undertaken in accordance with "Ethical Principles of Psychologists and Code of Conduct" provided by the American Psychological Association (APA) available at: http://www.apa.org/ethics/code/

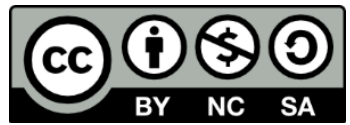

C) 2021. This work is licensed under a CC BY-NC-SA 4.0 International license.

This is an open access article distributed under the Creative Commons Attribution License which permits unrestricted use, distribution, and reproduction in any medium, provided the original work is properly cited. 


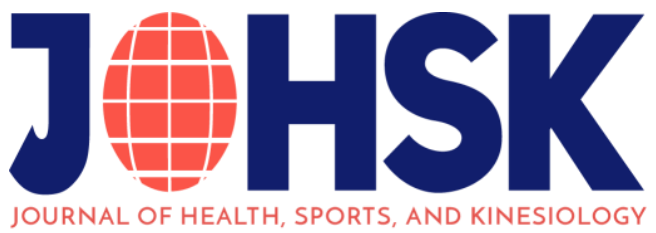

ISSN 2692-9864 (Online) \& ISSN 2767-5874 (Print)

\section{CONTENTS}

Keynote Address: "School Physical Education: Time to Make Good on Our Claims"

Hans van der mars..... 7

Maglala, An Occupational Dance of Badian, Province of Cebu

Mira Luna Panelag.

02 Exploring the Health Benefit of Taekwondo Intervention Among Nursing Home Residents

Yongseop Kim, Junhyung Kim, \& Dongchul Seo

Preparedness of Mind and Body of Athletes: Implications to Academic Performance

Angelo P. Guillermo

04 Athletes Perception on Parental Involvement

Hernando P. Diaz \& Estela F. Diaz.

05 A Drill-down Analysis of Psychological Well-Being among Asian Students from India, Thailand, and Vietnam: A Cross-sectional, Cross-cultural, Comparative Study Raul Calderon Jr.

06 Effects of Acute Different Intensities of Cycling Exercise on Blood Lipids and Lipoproteins in Sedentary Obese Men

Jinkyung Park \& Yunsuk Koh.

Coping Mechanisms of Teacher Education FIT Students in Online Based Learning Sheila Marie M. Marquez, Raigern Lornwilq S. Peralta, Tsude B. De Vera, Sophia Marie S. Liban, Wendy Mae D. Ulanday, Arsthein Naijle D.Fabia, Vincent Jr. C.Astudillo, Apple Mae M. Seculles, Cherry Ann U. Carranza, Jayvee Frazzen B. Laoyan, Klevin Joe J.Luquey

Acute Effect of Different Exercise Modalities on Working Memory and Motor Learning of a Golf Putting Task Chih-Chia Chen

Journal of Health, Sports, \& Kinesiology | ISSN 2692-9864 (Online) \& ISSN 2767-5874 (Print) | www.johsk.com 
Effects of Nutrition Knowledge on Dietary Behavior in Collegiate Students:

A Case Study Community

Emilija Visic, Suchi Prajapati, Yogesh Barhate, \& Soon-Mi Choi .....

Anthropometric Somatotypes in Youth in a Rural School District in Kansas

Skye Choi \& Young Sub Kwon

11 Leadership Traits and Self-Esteem of Selected Martial Arts Practitioners

in the University of Santo Tomas

Stewen R. Jain, Jan Michael Ezekiel E. Jaucian,

Zachary T. Huang, Michael David T. Lopez.

12 A Case Study: Teaching Materials Developments, Practices, and Applications of Using QR codes in Physical Education classes

Boung Jin Kang .....

Prospective Association of Handgrip Strength with Medical Utilization and the Risk of Hospitalization in Korean Adults Ju Seong Lee, Young Sub Kwon, Nam Hun Kim, Joo Eun Park,

Sin-Beom Kang, Jae-Yong Jang, \& Junghoon Kim

14 Resting Heart Rate is a Prognostic Factor for Mortality in Patients with Colorectal Cancer

Wonhee Cho, Justin Y. Jeon, \& Joon Young Kim

15 The Combination of Novel Biomarkers Identifies Individuals

of Heighten Risk for Type 2 Diabetes

Jared Rosenberg \& Joon Young Kim

16 Moderate-Load Muscular Endurance Descending Resistance Training did not

Improve 1RM in Resistance-Trained Men

Hyun Min Kim, Junghoon Kim, Min Hyuk Kwon, Yun Seo Choi, \& Young Sub Kwon.

17 Research Critique on "The Effects of Spirulina Under High-Intensity Interval Training on Levels of Nesfatin-1, Omentin-1, and Lipid Profiles in Overweight and Obese Females: A Randomized, Controlled, Single-Blind Trial"

Alyssa H. Shin, Ohjun Kwon, Seoyoung Moon, \& Young Sub Kwon

18 Research Critique on "Footwear Insoles with Higher Frictional Properties

Enhance Performance by Reducing In-Shoe Sliding During Rapid Changes of Direction"

Sydney Williams \& MinHyuk Kwon

Research Critique on "Influence of Sports Flooring and Shoes on Impact Forces and Performance During Jump Tasks"

Shannon Sung-Jin Kim \& MinHyuk Kwon. 48

Journal of Health, Sports, \& Kinesiology | ISSN 2692-9864 (Online) \& ISSN 2767-5874 (Print) | www.johsk.com 
20 Research Critique on "Effects of Finger Taping on Forearm Muscle Activation in Rock Climbers"

Sydney Snelbaker \& MinHyuk Kwon

21 Research Critique on "A Comparison of Velocity-Based and Traditional Percentage-Based Loading Methods on Maximal Strength and Power Adaptations" Casey J. Kimbro, Luis Medina, \& Young Sub Kwon

22 Research Critique on "Assessment of MTNR1B Type 2 Diabetes Genetic Risk Modification by Shift Work and Morningness-Eveningness Preference in the UK Biobank"

Nathan Redmond \& Joon Young Kim

23 Research Critique on "Effects of Time-Restricted Feeding in Weight Loss, Metabolic Syndrome and Cardiovascular Risk in Obese Women"

Sydney Davis \& Joon Young Kim.....

24 Research Critique on "Efficacy and Feasibility of HIIT

Training for University Students: The Uni-HIIT RCT"

Sewina Yu \& Joon Young Kim

25 Research Critique on "How Diet and Nutrition-Related Mobile Apps Influence Behavior" Vanessa Gonzalez, Eric Serrano, Francis Oppong, Richard Layos, \& Hosung So.

26 Research Critique on "Effects of Resistance Training on Tendon Mechanical Properties and Rapid Force Production in Prepubertal Children"

Humberto Magana, Mason Roach, Matthew Thoe, \& Hosung So

27 Research Critique on "Stereotypical Views of Beauty and Boys Still Not Letting Girls Play: A Student-Centered Curriculum for Young Girls Through an After-School Activist Approach" Michael Grimes, Cheyenne Wood, Ivan Magana, Moira O'Neill, \& Hosung So

Research Critique on "Parents and Children's Physical Activity Relationship"

Juan Carlos, Kassandra Villalobos, \& Hosung So

29 Research Critique on "Health-Related Physical Fitness and Physical Activity in Elementary School Students"

Bobby Wiley \& Hosung So

Research Critique on "Perspectives of Students with Disabilities Toward Physical Education: A Qualitative Inquiry Review" Katherine Walker, Christian Briceno, Shaun Cannady, \& Hosung So

Journal of Health, Sports, \& Kinesiology | ISSN 2692-9864 (Online) \& ISSN 2767-5874 (Print) | www.johsk.com 
31 Effects of Ketogenic Dietary Intervention on Anthropometrics, Body Composition, Metabolic Parameters, and Psychological Factors in Young Obese Population

Hayden West \& Soon-Mi Choi...

32 Effects of VR Games on Mental Health Among College Students: A Review of Literature

Se Ik Park, Eun-Ok Baek, \& Hosung So.

33 Physical Preparation for Junior Tennis Players

Alexandru Nicolae Banciu \& Germina Cosma......

34 The U.S. Insulin Crisis

Viktoria Layton, Priya Desai, \& Joon Young Kim 86

35 The Epidemiology of Well-Being, Happiness, Perceived Stress, and Health Behaviors of Asian University Students: A Multinational Project

Raul Calderon Jr. 88

Photo of 2021 October International Conference Presenters:

Keynote Speaker, Original Research Presentation, Undergraduate Student Research Critique, Graduate Student Research Grant Report, Ongoing Research Project. 


\title{
00 KEYNOTE PRESENTATION
}

\section{School Physical Education: Time to Make Good on Our Claims}

\author{
Dr. Hans van der Mars \\ Physical Education Teacher Education Program \\ Mary Lou Fulton Teachers College \\ Arizona State University, USA \\ Hans.Vandermars@asu.edu
}

\begin{abstract}
Biography
"Hans Van der Mars is a professor at Arizona State University and is actively involved in the undergraduate, master's and doctoral degree programs in physical education teacher educationsport pedagogy. Professor Van der Mars held a faculty appointment at Oregon State University for 15 years after previously holding faculty positions at Arizona State University (Tempe) and the University of Maine-Orono. He has been an active researcher in sports pedagogy/physical education teacher education for more than 30 years. Professor Van der Mars (co-) authored more than 100 published research papers, professional papers, book chapters, proceedings papers, and three textbooks. He has delivered 215 invited keynote, research and professional presentations at international, national regional and state level conferences. Professor Van der Mars regularly hosts workshops for K-12 teachers in physical education. He served as co-editor of the Journal of Teaching in Physical Education (JTPE) from 1998 to 2002. Professor Van der Mars is a research fellow in the Society of Physical and Health Educators of America (SHAPE America) and was inducted as an active fellow in the American Academy of Kinesiology and Physical Education (AAKPE) in 2006. He served as a member of the President's Council on Fitness, Sport and Nutrition Science Board for a three-year term. Professor Van der Mars' research focuses on determining factors that facilitate physical activity among secondary school students. Schools are viewed as a primary venue for influencing physical activity habits of children and youth, so it is important to determine which variables affect students' physical activity opportunities. This is especially important for those students who are not part of their school's interscholastic athletics program." Excerpted from https://education.asu.edu/about/people/hans-van-der-mars
\end{abstract}

\section{Presentation Outline}

1. Physical education: A marginalized school subject, and why?

2. Physical education in 1950 vs. 2020

3. Physical education's stiff competition

4. What NOT to do

5. What we CAN/MUST do: Create conditions for meaningful outcomes

6. Who is responsible for moving us forward?

7. Closing observations and conclusion

\section{References for Keynote October 2021}

Corbin, C. B. (2002). Physical activity for everyone: What every physical educator should know about promoting lifelong physical activity. Journal of Teaching in Physical Education, 21 (2), $128-$ 144. 
Pate, R. R., \& Hohn, R. C. (1994). Health and fitness through physical education. Human Kinetics.

Doyle, W. (1979). Classroom tasks and students' abilities. In P. L. Peterson \& H. J. Walberg (eds.), Research on Teaching: Concepts, Findings, and Implications (pp. 183-209). McCutchan.

Drape, J. (2018). The youth sports megacomplex comes to town, hoping teams will follow. New York Times.

Retrieved from https://www.nytimes.com/2018/09/12/sports/youth-sports/costs.html

Hardman, K. (2011). Global issues in the situation of physical education in schools. In K. Hardman \& K. Green (Eds.), Contemporary issues in physical education (pp. 11-29). Maidenhead: Meyer \& Meyer Sport.

Hardman, K. (2013). W(h)ither school physical education provision in Europe: two decades of research evidence. Journal of the International Federation of Physical Education, 83, 115-119.

Lounsbery, M. A., McKenzie, T. L., \& Thompson, H. R. (2019). Prioritizing physical activity in schools: Educational leaders' perspectives. Translational Journal of the American College of Sports Medicine, 4 (22), 248-256.

McCloy, C. H. (1938). In quest of skills. The Journal of Health and Physical Education, 9(8), 478-525.

Mitchell, M. F., Lawson, H. A., van der Mars, H., \& Ward, P. (2021). Chapter 9: Pathways toward desirable futures. Journal of Teaching in Physical Education, 40(3), 423-430. doi: https://doi.org/10.1123/jtpe.2020-0247

Sahlberg, P. (2021). Finnish Lessons 3.0: What Can the World Learn from Educational Change in Finland? (3rd ed.). Teachers College Press.

Sperka, L., \& Enright, E. (2018). The outsourcing of health and physical education: A scoping review. European Physical Education Review, 24(3), 349-371.

Trost, S., \& van der Mars, H. (2009). Why we should not cut PE. Educational leadership, 67 (4), 60-65.

UNESCO (2015). Quality Physical Education - Guidelines for policy-makers. Retrieved from https://en.unesco.org/inclusivepolicylab/sites/default/files/learning/document/2017/1/23110 1E.pdf

van der Mars, H., McNamee, J., \& Timken, G. (2018). Physical education meets teacher evaluation: Supporting secondary school physical educators in formal assessment of student outcomes. The Physical Educator, 75, 581-615. doi: https://doi.org/10.18666/TPE-2018-V75-14-8471

van der Mars, H., Timken, G., \& McNamee, J. (2018). Systematic observation of formal assessment of students by teachers (SOFAST). The Physical Educator, 75, 341-371. doi: https://doi.org/10.18666/TPE-2018-V75-13-8113

van der Mars, H. (in press). Charles H. McCloy Research Lecture - "You're Not in Kansas Anymore" by Charles H. McCLoy. Research Quarterly for Exercise and Sport.

van der Mars, H., Lawson, H. A., Mitchell, M. F., \& Ward, P. (2021). Chapter 2: Reversing policy neglect in U.S. physical education: A policy-focused primer with suggested tangible actions. Journal of Teaching in Physical Education, 40(3), 353-362. doi: https://doi.org/10.1 123/jtpe.2020-0240

Wilkins, J. L., Graham, G., Parker, S., Westfall, S., Fraser, R. G., \& Tembo, M. (2003). Time in the arts and physical education and school achievement. Journal of Curriculum Studies, 35, 721-734.

Williams, B. J., \& Macdonald, D. (2015). Explaining outsourcing in health, sport and physical education. Sport, Education and Society, 20(1), 57-72.

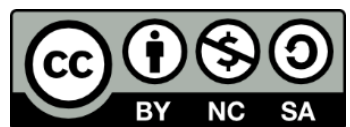

C 2021. This work is licensed under a CC BY-NC-SA 4.0 International license. This is an open access article distributed under the Creative Commons Attribution License which permits unrestricted use, distribution, and reproduction in any medium, provided the original work is properly cited. 


\title{
01 ORIGINAL RESEARCH
}

\section{Maglala, An Occupational Dance of Badian, Province of Cebu}

\author{
Mira Luna Panelag \\ University of Cebu, Philippines
}

Keywords: Maglala, Cebuano folk dance repertoire, weaving industry

\begin{abstract}
Introduction: Maglala (also called intertwine) is one of the main occupations in the Municipality of Badian in which the pandan leaves would serves as the source main materials for weaving a banig (mat). Weaving became a common and main occupation that dates back the Spanish Era. It was a tradition that was passed from generations to generations. With this honor, culture, and tradition from the ancestors of the occupants in Badian, the community created and developed a dance called Maglala. This dance was created because of its unique process, the movements used in the choreography are the ani (harvest) hagod (stretch) and lala (intertwine). This dance was created to value to the work of the people in Badian, which is the banig (mat). The people in Badian wanted to preserve their occupation for the next generation by creating the Maglala, initially an occupation into a dance.
\end{abstract}

Purpose: This study aims to document the Maglala, an occupational dance of Badian, Province of Cebu, the result which would be used to propose an action plan on the enrichment of the Cebuano folk dance repertoire.

Methods: In this study, the researcher used participant observation and descriptive analysis with the use of interview questioners. The researchers chose 11 respondents in Badian, Province of Cebu. Five for the dancers, five for the weavers, and one local founder of the Maglala, Mr. Mario Norrieto Apostol. The participants must be a resident of the area for more than 10 years and their age ranged is 18-80 years old. The researchers gathered the data from the informants with the use of an interview guide that inquires the basic steps, costumes, music, props, and the purpose of the Maglala, Participatory observation would be used to learn the dance choreography. Also, the researchers are required to weave and dance the Maglala. The data gathered via guide questions, videos, and pictures was interpreted through descriptive analysis.

Result: By using participatory observation, the researchers gathered additional movements through the process of making a banig. This movements could be an additional choreography or steps. The song's titled is "Ang kang Inday nga mga Saging", it has been considered as a Lullaby by women of Badian. The main purpose of the song was to make children sleep while the mothers are weaving a

Journal of Health, Sports, \& Kinesiology | ISSN 2692-9864 (Online) \& ISSN 2767-5874 (Print) | www.johsk.com 
| 2021 | Volume 2 | Issue 3(S) | The Journal of Health, Sports, and Kinesiology |

Banig. The song was supposed to be $4 / 4$-time signature, but it was changed to $3 / 4$ time signature to be suitable for dance rhythm. The data also implies the cultural and historical significance of weaving in Badian as a common occupation for more than centuries.

Conclusion: The Maglala is an occupational dance since it portrays the common work of the people which is intertwining pandanus strips that make-up the banig. It is very important to globally recognize Maglala as Type of Folk Dance because of its cultural heritage and value. This would help to preserve and promote Filipino culture for the next generation.

\section{Author Information}

Mira Luna Panelag, https://orcid.org/0000-0002-6703-6521

BSED Major in MAPEH, College of Teacher Education, University of Cebu, Main Campus, Philippines.

Email: miralunapanelag18@gmail.com

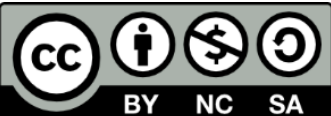

(c) 2021. This work is licensed under a CC BY-NC-SA 4.0 International license.

This is an open access article distributed under the Creative Commons Attribution License which permits unrestricted use, distribution, and reproduction in any medium, provided the original work is properly cited. 
| 2021 | Volume 2 | Issue 3(S) | The Journal of Health, Sports, and Kinesiology |

Journal of Health, Sports, and Kinesiology, 2021, 2(3), 11-12. https://doi.org/10.47544/johsk.2021.2.3

(c) 2020-2021 by the International Organization for Health, Sports, \& Kinesiology

\title{
02 ORIGINAL RESEARCH
}

\section{Exploring the Health Benefit of Taekwondo Intervention Among Nursing Home Residents}

\author{
Yongseop Kim, Junhyung Kim, \& Dongchul Seo \\ Indiana University, Bloomington, USA
}

Keywords: Taekwondo intervention, physical health, cognitive ability, social health, mental health, nursing home resident

\begin{abstract}
Introduction: Prior studies have shown that physical inactivity is prevalent among nursing home residents, and it has a direct influence on loss of lower body muscle mass, which increases the risk of falling for the elderly. Accumulative evidence suggests that participating in leisure-time physical activity increases physical functions, mental health, and cognitive abilities and provides social benefits for older adults. Taekwondo, a form of Korean traditional martial arts, can be an effective means for promoting health benefits among nursing home residents in the United States.
\end{abstract}

Purpose: Thus, the aim of this study was to explore the health benefits for nursing home residents of participation in a Taekwondo program modified to accommodate their physical and cognitive conditions.

Methods: Observational research method was incorporated to explore the health benefits of Taekwondo. Ten prospective participants ( 5 females, 5 males; mean age $=80$ years) from the local nursing home facility participated in in-depth semi-structured interviews. The Taekwondo program consisted of 8 weeks of basic movements, including stances, punching, blocking, and kicking. The program was implemented by a certified Taekwondo instructor with volunteers. The researchers used a grand-tour and mini-tour question strategy guided by Strauss and Corbin (1998) and followed Creswell's (2009) five steps of data collection and analysis. For the data analysis, the constant comparative method was used for this study.

Results: Four main themes were identified as health benefits resulting from Taekwondo participation: (a) promoting mental health, (b) increasing physical functions, (c) stimulating cognitive abilities, and (d) facilitating positive social interaction. This study indicates Taekwondo can be instrumental in promoting their physical functioning, cognitive functioning, social interactions, and mental health.

Conclusions: The results of this study indicate that Taekwondo can be used as an intervention to increase the physical activity participation and ameliorate negative psychological consequences associated with confinement to nursing homes. In particular, Taekwondo is significantly associated

Journal of Health, Sports, \& Kinesiology | ISSN 2692-9864 (Online) \& ISSN 2767-5874 (Print) | www.johsk.com 
| 2021 | Volume 2 | Issue 3(S) | The Journal of Health, Sports, and Kinesiology |

with Korean cultural components that provide intellectual stimulation that can motivate participation. Overall, this study suggests that providing the invigorating bodily engagement in Taekwondo in a group format makes it a vehicle for improving physical, social, emotional, and cognitive functioning among nursing home residents.

\section{Author Information}

Yongseop Kim, https://orcid.org/0000-0001-5689-9340

Ph.D. student, School of Public Health, Indiana University, Bloomington, IN, USA.

Email: yk67@iu.edu

Junhyung Kim, Ph.D.,

Assistant Professor, School of Public Health, Indiana University, Bloomington, IN, USA.

Email: kim9@iu.edu

Donchul Seo,

School of Public Health, Indiana University, Bloomington, IN, USA.

Email: seo@indiana.edu

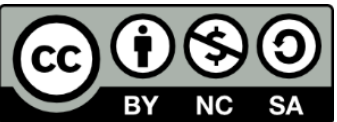

(C) 2021. This work is licensed under a CC BY-NC-SA 4.0 International license.

This is an open access article distributed under the Creative Commons Attribution License which permits unrestricted use, distribution, and reproduction in any medium, provided the original work is properly cited. 
Journal of Health, Sports, and Kinesiology, 2021, 2(3), 13-14. https://doi.org/10.47544/johsk.2021.2.3

( ) 2020-2021 by the International Organization for Health, Sports, \& Kinesiology

\title{
03 ORIGINAL RESEARCH
}

\section{Preparedness of Mind and Body of Athletes: Implications to Academic Performance}

\author{
Angelo P. Guillermo \\ Tarlac Agricultural University, Philippines
}

Keywords: body and mind preparedness, athlete, academic performance

\begin{abstract}
This study was conducted to determine the level of preparedness of athletes' body and mind along with physical, social, mental and emotional state and their relationship to their academic performance. Results revealed that in terms of level of preparedness of body and mind of athletes, they believed that they are physically and mentally prepared. However, they were only moderately prepared emotionally and socially. In terms of the differences between male and female athletes with their physical state, there is no significant difference between male and female athletes since the probability value $(p=.7655)$ is greater than 0.05 . With regards to athlete's level of readiness on mental state, male athletes are more stable or ready in terms of their mental condition than the female athletes. As to emotional state, female athletes are more prepared than the male athletes in terms of their emotional condition. With regards to social state, the level of preparedness on social state or condition of male and female athletes is the same or comparable. Moreover, there is a significant moderate relationship between the physical condition of athletes and their academic performance. The level of physical readiness of athlete becomes higher; it follows that his or her academic performance becomes better as well. With regards to the relationship between mental state and academic performance of athletes, academic performance of athletes becomes better when the athletes' level of mental preparedness increases. In terms of the relationship of emotional condition and academic performance of athletes, the academic performance of athletes tends to be high when their level of emotional preparedness is high as well. However, there is no significant relationship between the academic performance of athletes to their level of social preparedness since the probability value is greater than $0.05(p=0.241)$. Additionally, on the bases of the findings and conclusions of the study, the following are recommended: (a) since the athletes are only moderately prepared emotionally and socially, the coaches and athletes may formulate a functional action plan on how to enhance or improve their emotional and social conditions, (b) since physical, mental and emotional preparedness of athletes are found to correlates of academic performance, studentathletes must endeavor to maintain and further enhance their being physically, mentally and emotionally fit, (c) similar studies may be conducted making use of other variables to be correlated to the body and mind conditions of the athletes, (d) the copy of the results of the study may consider
\end{abstract}

Journal of Health, Sports, \& Kinesiology | ISSN 2692-9864 (Online) \& ISSN 2767-5874 (Print) | www.johsk.com 
| 2021 | Volume 2 | Issue 3(S) | The Journal of Health, Sports, and Kinesiology |

providing the concerned offices/department of TAU and TSU to serve as basis in enhancing the body, mind and academic performance of their athletes, $(e)$ to include preparation of the mind in the sports development program of the schools and Universities, (f) coaches and trainers should undergo trainings and seminars regarding this concern with the full support of the administration, and (g) faculty members handling student-athletes must also be informed about these findings.

\section{Author Information}

Angelo P. Guillermo, https://orcid.org/0000-0001-5563-5415

Physical education teacher, Tarlac Agricultural University, Philippines.

Email: aguillermo@tau.edu.ph

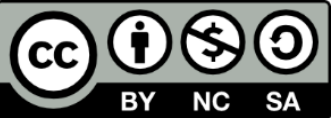

(C) 2021. This work is licensed under a CC BY-NC-SA 4.0 International license.

This is an open access article distributed under the Creative Commons Attribution License which permits unrestricted use, distribution, and reproduction in any medium, provided the original work is properly cited. 
| 2021 | Volume 2 | Issue 3(S) | The Journal of Health, Sports, and Kinesiology |

Journal of Health, Sports, and Kinesiology, 2021, 2(3), 15-16. https://doi.org/10.47544/johsk.2021.2.3

( ) 2020-2021 by the International Organization for Health, Sports, \& Kinesiology

\title{
04 ORIGINAL RESEARCH
}

\section{Athletes Perception on Parental Involvement}

\author{
Hernando P. Diaz \& Estela F. Diaz
}

Universidad de Manila, Philippines

Keywords: athletes, parents, enjoyment

\section{ABSTRACT}

Introduction: Every year, athletes in almost all schools are becoming highly competitive because of their preparation in extramural sports. Educational institutions joined group of schools to have game with. Hiring highly competitive coaches and trainers to make the athletes prepared for competitions. Because of the championship goal, athletes treated like robots, like a non-tiring individual every practice, doing impossible skills to be possible. Not considering other factors that very important to athletes' world of sport, like the influence of their loved ones, the family - the parents - the father and mother that have big impact in athletes' life. In addition, most importantly, the athlete's self, their feelings, their enjoyment in sport!

Purpose: The purpose of this study was to explore the athletes' perception of parental involvement and enjoyment in their sport in a public university in Manila in School Year 2018-2019.

Methods: The respondents included 120 athletes in different sports who completed the Parental Involvement Scale Questionnaire (PISQ) and Physical Activity Enjoyment Scale (PACES). The four scale of PISQ: Directive Behavior, Praise and Understanding, Active Involvement and Parental Pressure were used to assess perception of the involvement of their parents in sport according to the different variables (age, gender, only child, with siblings, order of birth and parent involved). The PACES includes a set of 18 items seven point bipolar scale (e.g. "I enjoy it"..."I hate it"...). Descriptive statistics, ANOVA, paired t-test, and Pearson Correlation were used to analyze the results.

Results: The results of this study indicated that in general, fathers were perceived as more directive and active in their child's sport while mother show care, praise and understanding. Athletes in general enjoy sport. Parents were perceived to become more directive and active involved to their sons. However, parents were more sympathetic and understanding to their daughters. Male athletes also enjoy their sports more. Parents of only children were perceived as more directive, understanding and pressuring while children with siblings perceived their parents as more actively involves. Only children also enjoy their sport more than those who have siblings. First and last children perceived their parents as more involved in their sport than middle children. Only fathers are more involved in their children's

Journal of Health, Sports, \& Kinesiology | ISSN 2692-9864 (Online) \& ISSN 2767-5874 (Print) | www.johsk.com 
| 2021 | Volume 2 | Issue 3(S) | The Journal of Health, Sports, and Kinesiology |

sport than with only mothers, but athletes whose parents are both involved enjoyed more in their sports than those who's neither of the parents were involved. Using Pearson Correlation, a mother's parental pressure and a father's directive behavior and active involvement has an effect on athletes' enjoyment in sport.

Conclusions: The research gave information on coaches the effect of parental involvement of parents in their athletes' performance and to the parents, the right parenting style to apply in their child for the improvement of the sporting community

\section{Author Information}

Hernando P. Diaz, https://orcid.org/0000-0003-3389-0831

Department of Physical Education, College of Education, Universidad de Manila, Philippines.

Email: dingdiaz00@yahoo.com

Estela F. Diaz, https://orcid.org/0000-0001-5220-4839

Department of Physical Education, College of Education, Universidad de Manila, Philippines.

Email: esteladz@yahoo.com

(c) (1) (8) (2)

(C) 2021. This work is licensed under a CC BY-NC-SA 4.0 International license.

This is an open access article distributed under the Creative Commons Attribution License which permits unrestricted use, distribution, and reproduction in any medium, provided the original work is properly cited. 


\title{
05 ORIGINAL RESEARCH
}

\section{A Drill-down Analysis of Psychological Well-Being Among Asian Students from India, Thailand, and Vietnam: A Cross-sectional, Cross-cultural, Comparative Study}

\author{
Raul Calderon Jr. \\ Former Visiting Professor, Faculty of Sports Science, Kasetsart University, Thailand
}

Keywords: psychological well-being, students, cross-cultural comparisons

\begin{abstract}
Introduction: Research on psychological well-being (PWB) has increased over the last 3 decades. Results from multicultural studies have reported significant gender, age, and group differences in PWB among subjects from different countries/cultures. However, there are major limitations common among these multicultural studies, i.e., (1) a lack of a well-defined or operationalized construct for PWB, (2) in-depth cross-cultural comparisons in levels of PWB, and (3) limited data on student populations.
\end{abstract}

Purpose: This study aimed: (1) to use a six-dimension theory-based model of PWB to describe levels of PWB in Asian university students and (2) compare differences in PWB among Asian university students from India, Thailand, and Vietnam.

Methods: A cross-sectional research design was applied with undergraduate students $\left(1^{\text {st }}, 2^{\text {nd }}, 3^{\text {rd }}\right.$, and $4^{\text {th }}$ year) from India $(N=508)$, Thailand ( $N=1132$ ), and Vietnam ( $\left.N=1821\right)$. The six universities (1 from India, 2 from Thailand, and 3 from Vietnam) ranged in size (smallest $<1000$ and largest $>10,000$ ), type (private and public), and specialization (public health, sports, humanities, and technology). Students completed an online or paper survey in a classroom setting from October 2016-December 2017. The survey included demographic questions and the 54-item Ryff scales of psychological well-being (RSPWB), which consists of six subscales (1. autonomy, 2. environmental mastery, 3. purpose in life, 4. positive relations, 5. personal growth, and 6. self-acceptance), with 9-items per scale, and contains a six-point Likert scale (from 1=Strongly Disagree to $6=$ Strongly Agree). A composite PWB score (sum of all subscales) was also calculated for each country. Data were analyzed using univariate, bivariate, ANOVA, and Post Hoc statistics on SPSS (2019).

Results: The highest and lowest subscale scores for each country are as follows: India, autonomy $(X=33.92, S D=5.58)$-personal growth $(X=30.73, S D=4.97)$; Thailand, positive relations $(X=37.67, S D=6.72)$ autonomy $(X=32.86, S D=4.84)$; Vietnam, self-acceptance $(X=33.88, S D=4.60)$-purpose in life $(X=32.20$,

Journal of Health, Sports, \& Kinesiology | ISSN 2692-9864 (Online) \& ISSN 2767-5874 (Print) | www.johsk.com 
$S D=5.70)$. Thailand had the highest composite PWB score $(X=215.71, S D=25.10)$ and India the lowest $(X=196.36, S D=16.81)$. ANOVA and Post Hoc results showed that Thailand had significantly higher subscale and PWB scores ( $p<0.05)$ compared to Vietnam and India in all subscales, except autonomy. India had a significantly higher subscale score $(p<0.05)$ in autonomy compared to Thailand and Vietnam. Overall, Thailand had the highest subscale scores followed by Vietnam and then India.

Conclusion: Based on the analysis, we conclude that scores on the six dimensions (autonomy, environmental mastery, purpose in life, positive relations, personal growth, and self-acceptance) of the RSPWB vary in strength and rank in university students from India, Thailand, and Vietnam. Moreover, the data also indicates that significant differences exist in the six dimensions and composite scores of PWB between university students from the three countries studied.

Discussion: To the best of our knowledge, this is the first in-depth cross-cultural study on PWB among university students from India, Thailand, and Vietnam using a theory-based construct to document variations and patterns in PWB and its corresponding six dimensions. The results indicate that the relative strength and rank/order of the six dimensions (autonomy, environmental mastery, purpose in life, positive relations, personal growth, and self-acceptance) varies within Asian counties. Moreover, as importantly, the results further indicate that the strength and rank/order of PWB and its corresponding six dimensions also varies by country. These findings have important implications, i.e., (1) descriptive data from this study can be used for comparative purposes by researchers conducting cross-cultural research, (2) individual significance of the six dimensions of PWB can be further studied to determine the role that each dimension plays, if any, in the overall development of students, (3) develop statistical models to test potential factors responsible for the PWB differences among students and countries, and (4) develop and test intervention strategies to increase/improve the PWB of university students. One limitation of this study is that the population was restricted to undergraduate students, thus these findings may not generalize to other adult populations.

\section{Author Information}

Raul Calderon Jr. Ph.D., https://orcid.org/0000-0002-7580-3057

Former Visiting Professor, Faculty of Sports Science, Kasetsart University, Thailand \& Executive Advisor, International Organization of Health, Sports, and Kinesiology (IOHSK), USA.

Email: raulthedoc@gmail.com

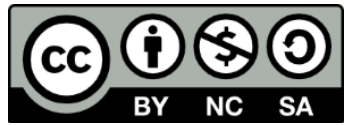

(C) 2021. This work is licensed under a CC BY-NC-SA 4.0 International license.

This is an open access article distributed under the Creative Commons Attribution License which permits unrestricted use, distribution, and reproduction in any medium, provided the original work is properly cited. 


\title{
06 ORIGINAL RESEARCH
}

\section{Effects of Acute Different Intensities of Cycling Exercise on Blood Lipids and Lipoproteins in Sedentary Obese Men}

\author{
Jinkyung Park' \& Yunsuk Koh² \\ 1 Georgia College \& State University, Milledgeville, GA, USA \\ 2 Baylor University, Waco, TX, USA
}

Keywords: obesity, exercise intensity, lipid, cholesterol

\begin{abstract}
Introduction: With the increasing prevalence of obesity in our society, a consistent correlation between obesity and the likelihood of cardiovascular disease has become evident. Cardiovascular disease, in particular, can include high blood pressure, heart attack, stroke, heart failure, or arrhythmia. These conditions can be a byproduct of atherosclerosis-a build-up of plaque in the arteries-or blood clots in the heart or brain due to abnormal blood lipids and lipoproteins. It is unanimously agreed upon in the healthcare community that exercise can mitigate and reduce high levels of atherosclerosis, high levels of cholesterol and triglycerides, and balance the lipid profile. However, it is unclear which intensity of exercise yields the most effective results. Previous studies on the effectiveness of high-, moderate-, and low-intensity exercise on lipid levels have reported significantly variable results due to different healthy populations.

Purpose: This study aimed to examine the response of blood lipid and lipoprotein levels following different intensities of cycling exercise in sedentary obese males.

Methods: Fifteen obese (body mass index $>30 \mathrm{~kg} / \mathrm{m}^{2}$ ), sedentary (less than 2 days per week of physical activity) males, aged 18-30 years, participated in this randomized, cross-over study. The study was reviewed and approved by the University Institutional Review Board for human subjects, and all subjects signed informed consent documents prior to testing. The participants performed a single bout of cycling exercise (average energy expenditure: $300 \mathrm{kcal}$ ) at two different intensities [lower-intensity: $50 \%$ of maximal heart rate and higher-intensity: $80 \%$ of maximal heart rate] in a random order. All participants recorded their food intake over 3 consecutive days (from 2 days prior to exercise to the day of exercise) for each exercise trial. Overnight fasting blood samples were collected at baseline, immediately post-exercise (IPE), 1-hr PE, and 24-hr PE for each intensity of exercise to determine the profile of blood lipids and lipoproteins (TC, TG, LDL-C, and HDL-C). A 2 (intensity) $\times 4$ (time) analysis of
\end{abstract}

Journal of Health, Sports, \& Kinesiology | ISSN 2692-9864 (Online) \& ISSN 2767-5874 (Print) | www.johsk.com 
variance with repeated measures was used to examine the main and interaction differences in intensity and time on the profile of blood lipids and lipoproteins $(p<.05)$.

Results: Total calories and macronutrient contents (fat, carbohydrate, and protein) between the two exercise trials were not significantly different. The blood lipids and lipoproteins were not significantly altered following either lower or higher intensity exercise. There was no significant interaction between intensity and time.

Conclusion: The results suggest that regardless of exercise intensity, an acute bout of aerobic exercise requiring $300 \mathrm{kcal}$ energy expenditure may not be enough to significantly alter blood lipids and lipoproteins in physically healthy obese males. Therefore, it is recommended that future research determine whether different intensities of chronic exercise requiring the same or higher volume of energy expenditure can positively alter the blood lipid profiles in obese males.

\section{Author Information}

Jinkyung Park, Ph.D., https://orcid.org/0000-0003-4888-8881

Assistant Professor, Department of Exercise Science, School of Health and Human Performance, Georgia College \& State University, Milledgeville, GA, USA.

Email: inkyung.park@gcsu.edu

Yunsuk Koh, Ph.D. https://orcid.org/0000-0001-9280-5786

Associate Professor \& Director of Exercise Physiology, Department of Health, Human Performance, and Recreation, College of Health and Human Sciences, Baylor University, Waco, TX, USA.

Email: yunsuk koh@baylor.edu 


\title{
07 ORIGINAL RESEARCH
}

\section{Coping Mechanisms of Teacher Education FIT Students in Online Based Learning}

\author{
Sheila Marie M. Marquez, Raigern Lornwilq S. Peralta, Tsude B. De Vera, \\ Sophia Marie S. Liban, Wendy Mae D. Ulanday, Arsthein Naijle D.Fabia, \\ Vincent Jr. C.Astudillo, Apple Mae M. Seculles, Cherry Ann U. Carranza, \\ Jayvee Frazzen B. Laoyan, Klevin Joe J.Luquey, \\ Saint Lovis University, Philippines
}

Keywords: coping, teacher education, online-based learning

\begin{abstract}
Introduction: A novel coronavirus (SARS-CoV-2, formerly known as 2019-nCoV) caused the latest COVID-19 pandemic which began in China and almost affected every country in the whole world is one of the most recent public health crises of global concern (Guo et al. 2020). The spread of COVID 19 has affected the educational system worldwide in particular, on the learning delivery (Toquero, 2020). Due to COVID19 pandemic, countries temporarily interrupted formal education activities in several fields and turned to the distance education method to sustain educational activities in countries that were affected, through the transitioning of course content and related materials to the digital world. Currently, the newest and most popular form of distance education worldwide is the "online-based learning", the type of instruction mediated via the internet and a term to describe an emerging approach to learn at students' own premise through advanced informationcommunication technologies which can be through synchronous or asynchronous instructions (Yang, 2020). It also covers a wide range of processes and applications, such as web-based learning, computer-based learning, virtual classrooms, and digital collaboration. This includes the delivery of content via the Internet, LAN / WAN (intranet / extranet), audio and videotape, satellite broadcasting, interactive TV, and CD-ROM (Paulsen, 2002). Today, education industries are adopting the technologies available such as digital Video conferencing platforms like Zoom, Microsoft platform, and Webex Blackboard and Google Classroom to enhance online learning globally (Larry 2020). The emergence of this novel coronavirus pandemic brought challenges in the field of education which resulted in the implementation of Online Based Learning (OBL) as one of the learning modalities in the Philippines. However, this learning modality comes with concerns and difficulties that should be addressed, especially to the students who enrolled in OBL.
\end{abstract}

Journal of Health, Sports, \& Kinesiology | ISSN 2692-9864 (Online) \& ISSN 2767-5874 (Print) | www.johsk.com 
Purpose: This research aims to explore the challenges encountered by Fundamental Integrative Training, particularly Physical Activity toward Health and Wellness- Outdoor and Adventure Activities (FIT-OA) course students at the School of Teacher Education, Saint Louis University, Baguio City, and their corresponding coping mechanisms in an Online Based Learning.

Methods: The FIT students who are currently enrolled in online based learning who are willing to participate were the participants of the study. For the administration of the interview, the participants would be informed in advance and guided accordingly. A qualitative phenomenological type of research was utilized in gathering and interpreting the data of the study since it aims to elucidate the meaning and structure as well as the essence of the lived experiences of a person, or a group of persons around a specific phenomenon. The experiences relating to this study were the ones that were thematized as the coping mechanisms of teacher education FIT students in online-based learning.

Results: After careful analysis of the data and audio recorded interviews from the six (6) participants, there were five (5) significant themes that emerged as challenges and another five (5) significant themes that emerged as coping mechanisms of FIT-OA students in online based learning. The themes for challenges were summarized as TEENS which stands for Technical Difficulties, Emotional and Mental Issues, Elaboration on lessons is lacking, non-conducive learning environment and Students Lack Discipline. On the other hand, the themes for coping mechanisms were summarized as STORM which stands for Students Fosters Resourcefulness, Time management, Optimistic Attitude, Rest, and Motivation. Results show that despite the challenges that FIT-OA students encountered in online based learning they overcome it with their coping mechanisms.

Conclusion: The result of the study can be a source of data and strategies by other students who are also having difficulties during online classes. Moreover, it can also be a guide for the teachers to sympathize and help improve their students' performance despite the difficulties. It can also be used to develop their teaching learning and evaluating strategies in enhancing effective learning process, skills, and attitudes to their students during the online learning.

\section{Author Information}

\section{Sheila Marie M. Marquez,}

Professor, Department of Physical Education, Saint Louis University, Philippines.

Email: smmagat@slu.edu.ph

Raigern Lornwilq S. Peralta, https://orcid.org/0000-0003-3115-8751

Department of Physical Education, Saint Louis University, Philippines.

Email: raigernhunhan0794@gmail.com

Tsude B. De Vera, https://orcid.org/0000-0002-3124-4097

Department of Physical Education, Saint Louis University, Philippines.

Email: 2190690@slu.edu.ph

Journal of Health, Sports, \& Kinesiology | ISSN 2692-9864 (Online) \& ISSN 2767-5874 (Print) | www.johsk.com 
Sophia Marie S. Liban, https://orcid.org/0000-0002-5475-3273

Department of Physical Education, Saint Louis University, Philippines.

Email: 2196512@slu.edu.ph

Wendy Mae D. Ulanday, https://orcid.org/0000-0001-5058-2545

Department of Physical Education, Saint Louis University, Philippines.

Email:wendymae061913@gmail.com

Arsthein Naijle D. Fabia, https://orcid.org/0000-0001-7929-5603

Department of Physical Education, Saint Louis University, Philippines.

Email: 2191356@slu.edu.ph

Vincent Jr. C. Astudillo, https://orcid.org/0000-0002-4413-0929

Department of Physical Education, Saint Louis University, Philippines.

Email: 2191306@slu.edu.ph

Apple Mae M. Seculles,

Department of Physical Education, Saint Louis University, Philippines.

Email: 2193018@slu.edu.ph

Cherry Ann U. Carranza,

Department of Physical Education, Saint Louis University, Philippines.

Email:

Jayvee Frazzen B. Laoyan, https://orcid.org/0000-0002-4263-359X

Department of Physical Education, Saint Louis University, Philippines.

Email: jvlaoyan@gmail.com

Klevin Joe J.Luquey,

Department of Physical Education, Saint Louis University, Philippines.

Email:

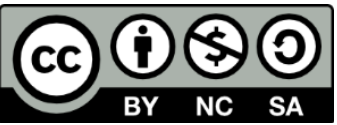

(C) 2021. This work is licensed under a CC BY-NC-SA 4.0 International license.

This is an open access article distributed under the Creative Commons Attribution License which permits

unrestricted use, distribution, and reproduction in any medium, provided the original work is properly cited. 
| 2021 | Volume 2 | Issue 3(S) | The Journal of Health, Sports, and Kinesiology |

Journal of Health, Sports, and Kinesiology, 2021, 2(3), 24-25. https://doi.org/10.47544/johsk.2021.2.3

() 2020-2021 by the International Organization for Health, Sports, \& Kinesiology

\title{
08 ORIGINAL RESEARCH
}

\section{Acute Effect of Different Exercise Modalities on Working Memory and Motor Learning of a Golf Putting Task}

\author{
Chih-Chia Chen \\ Mississippi State University, USA
}

Keywords: physical activity, working memory, golf putting

\begin{abstract}
Background: Several studies so far have demonstrated that physical activity could lead to improvements in executive function and performance. To date, much of the work in this area has been built on the influence of acute bouts of physical activity. This study was going to compare the effects of skill random practice and acute cardiovascular exercise on visuospatial working memory (VSWM) and golf putting performance.

Method: 24 healthy adults (10 males, 14 females, aged $21.02 \pm 0.70$ years) with no golf experience participated in the study. A set of baseline measures was administered, including two VSWM tests (i.e., memory matrix and rotation matrix) and a 10-ft. golf putt task. Then, a 30-min intervention was implemented after the baseline period. Participants were randomized into two exercise groups: skill random practice and acute cardiovascular exercise. The skill random group practiced 3 blocks of 3 feet, 6 feet, and 9 feet putts. Each block had 10 trials of putting, so the total number of practice trials was 90. The acute cardiovascular exercise group participated in a 30-min treadmill exercise, maintaining their heart rate between $65 \%$ and $85 \%$ of their predicted maximum heart rate (HRmax). An acquisition measure after the intervention and a 24-hour retention measure were given. Data were analyzed by separate analyses of 3 (time periods) $\times 2$ (groups) ANOVA.
\end{abstract}

Results: The scores in working memory tests were improved immediately after the intervention; however, the improvement in retention effect of VSWM was not maintained. Inconsistent with our findings in moderate-intensity exercise, the advantageous effect in 24-hour retention seemed to be noted in the high-intensity acute exercise studies. Therefore, more studies are still needed to differentiate the mediation effect of intensity of exercise on working memory. In addition, golf putts were seen to improve 24 hours after practice compared to the baseline. It could be possible that the effect of the underlying fatigue in the low extremities on the treadmill may hinder the short-term recall of the motor memory and thus mask the movement regulation of upper extremities (i.e., golf grip, arm swing) later in the golf putting task. The coexistence of facilitative and hindering mechanisms from walking might explain why exercise did not enhance motor skill acquisition immediately. Moreover, no

Journal of Health, Sports, \& Kinesiology | ISSN 2692-9864 (Online) \& ISSN 2767-5874 (Print) | www.johsk.com 
group difference was noted between skill random practice and acute cardiovascular exercise during acquisition and 24-hour retention tests. While performing skill random practice in golf putting, participants spent much time practicing fine and gross motor coordination, such as visual search, eyehand coordination, balance, and spatial orientation. These motor abilities demand higher-level cognitive processes and are likely to be related to attention and managing visual and spatial information that resulted in positive effects. The present study provides a proper level of contextual interference that would offer participants the opportunity to make adjustments during practice and may be beneficial for learning motor skills.

Conclusion: Although the results suggested several promising findings, adding a longer duration of retention test, such as a 7 -day retention test, would enable subsequent studies to investigate this area of interest more thoroughly. The present study is one of the pioneering studies that has attempted to pair acute cardiovascular exercise, executive function, and complex motor skill learning. The evidence suggested different temporal effects on VSWM and golf putting learning. The implication of this study is to address the importance of daily physical activity for facilitating better learning and memory for young students, as well as motor rehabilitation for older adults.

\section{Author Information}

Chih-Chia Chen, Ph.D., https://orcid.org/0000-0001-6963-3795

Associate Professor, Department of Kinesiology, Mississippi State University, USA.

Email: cc2196@msstate.edu

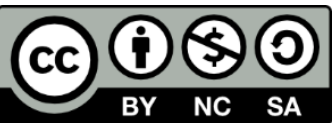

(C) 2021. This work is licensed under a CC BY-NC-SA 4.0 International license.

This is an open access article distributed under the Creative Commons Attribution License which permits unrestricted use, distribution, and reproduction in any medium, provided the original work is properly cited. 
| 2021 | Volume 2 | Issue 3(S) | The Journal of Health, Sports, and Kinesiology |

Journal of Health, Sports, and Kinesiology, 2021, 2(3), 26-27. https://doi.org/10.47544/johsk.2021.2.3

() 2020-2021 by the International Organization for Health, Sports, \& Kinesiology

\title{
09 ORIGINAL RESEARCH
}

\section{Effects of Nutrition Knowledge on Dietary Behavior in Collegiate Students: A Case Study}

\author{
Emilija Visic, Suchi Prajapati, Yogesh Barhate, \& Soon-Mi Choi \\ Midwestern State University, TX, USA
}

Keywords: nutrition knowledge, dietary behavior

\begin{abstract}
Introduction: The abandonment of traditional lifestyles and the increasing burden of cardiovascular disease are increasing global health problems. Diet education and educating about the macro and micro-nutrients, aim to delay, avoid or reduce the prevalence of these chronic and life-threatening conditions. According to Centers for Disease Control and Prevention guidelines, in the United States, $19 \%$ of young people aged 2 to 19 years and $40 \%$ of adults have obesity, which can put them at risk for heart disease, type 2 diabetes, and some cancers. Most college students do not have an adequate diet to lead healthy lives in the future because of a lack of education in nutrition and a lack of time to prepare or cook their own meals. It may result in eating fast foods or quick-frozen meals that do not provide proper nutrients.

Purpose: The purpose of study was to analyze how nutrition knowledge through an online nutrition course would affect dietary behavior in collegiate students.

Methods: The data collected from a nutrition course in collegiate level and four students (age $21.5 \pm 2$ yrs, height $171.5 \pm 5 \mathrm{~cm}$, weight $68.4 \pm 22.6 \mathrm{~kg}$ ) were volunteered and participated in three trials. All food and beverages intakes were tracked for three days (two weekdays and one weekend) per week and analyzed by NutritionCalc Plus (NCP) which is a dietary self-assessment tool and analysis software program (version 5.0, ESHA Research, Oak Brook, IL) during three weeks. This application allowed for the individual to input their physical data, such as height, weight, age, gender, and fitness level. When tracking their diet, the participants had to search through the database in NCP to identify all food and beverages which were distinguished as either breakfast, lunch, dinner, or snack. After all, three days had been completed in each week, the individual could download their weekly report, in which their macro and micronutrients were compared to the daily recommended intake (DRI) goal, based on their age, gender, and fitness level. The $1^{\text {st }}$ trial $(\mathrm{T} 1$ ) tracked food and beverages consumption and showed a baseline which was no feedback to food choice or consumption at the first week. The $2^{\text {nd }}$ trial (T2) and the $3^{\text {rd }}$ trial (T3) tracked food and beverages consumption at the second and the third
\end{abstract}

Journal of Health, Sports, \& Kinesiology | ISSN 2692-9864 (Online) \& ISSN 2767-5874 (Print)| www.johsk.com 
week, respectively, and the results were reflected by feedback and comments from the instructor. The consumption (\%) of nutrients based on an individual's DRI goal were analyzed as variables.

Results: Daily calorie intake (T1:72\%, T3:77.5\%), carbohydrate (T1: 197.5\%\%, T3: 88\%), fat (T1:69.75\%, T3:82.75\%), vitamin B1 (T1: 94\%, T3: 88.5\%), Potassium (T1: 76.25, T3: 63.5\%), and zinc (T1:78\%, T3: 94.25\%) were shown to maintain or meet within DRI recommended range after 3 weeks. However protein (T1: 155.75\%, T3:197.5\%), cholesterol (T1: 87.75\%, T3: 118\%), vitamin B3 (T1:115.75\%, T3:187\%), vitamin B6 (T1:114.25, T3:244.5\%), vitamin B12(T1: 100.5\%, T3: 254.25\%), vitamin B2 (T1: 111\%, T3:100.75\%), phosphorous (T1:98\%, T3: 117.5\%), iron (T1: 146.75, T3: 128.75\%), and sodium (T1: 63.5\%, T3: 141.5\%) exceeded the DRI recommendation. The intakes of dietary fiber (T1: 93.25\%, T3: 51.25\%), water (T1: 44.25\%, T3:40.5\%), vitamin D (T1: 28.25\%, T3: 59.25\%), folate (T1:65.75\%, T3:55.75\%), calcium (T1:63.25\%, T3: $64.75 \%$ ), and magnesium (T1: 128.75\%, T3:48\%) were decreased or lower than the DRI recommendation after 3 weeks.

Conclusion: In conclusion, following a nutrition education course, there were only certain specific changes made to intake of micronutrients. When looking at vitamin D and water intake, it was decreasing or lower than the DRI, while protein and sodium were exceeding the recommended intake. This shows that while an education course on nutrition may contribute slightly to a change in diet, it is not a general guide for most collegiate students. There could be more important factors that are influencing diet that outweigh the information being learned.

\section{Author Information}

Emilija Visic, https://orcid.org/0000-0002-7138-9629 Email: emivisic@gmail.com

Department of Athletic Training \& Exercise Physiology, Robert D. \& Carol Gunn College of Health Science \& Human Services, Midwestern State University, Wichita Falls, TX, USA.

Suchi Prajapati, https://orcid.org/0000-0003-3696-7128 Email: suchiprajapati20@gmail.com Department of Athletic Training \& Exercise Physiology, Robert D. \& Carol Gunn College of Health Science \& Human Services, Midwestern State University, Wichita Falls, TX, USA.

Yogesh Barhate, https://orcid.org/0000-0002-8105-9359

Department of Athletic Training \& Exercise Physiology, Robert D. \& Carol Gunn College of Health Science \& Human Services, Midwestern State University, Wichita Falls, TX, USA.

Email: yogeshbarhate17107@gmail.com

Soon-Mi Choi, Ph.D., https://orcid.org/0000-0002-2796-2061

Associate Professor, Department of Athletic Training \& Exercise Physiology, Robert D. \& Carol Gunn College of Health Science \& Human Services, Midwestern State University, Wichita Falls, TX, USA.

Email: soonmi.choi@msutexas.edu

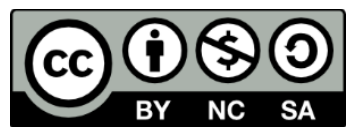

2021. This work is licensed under a CC BY-NC-SA 4.0 International license.

This is an open access article distributed under the Creative Commons Attribution License which permits unrestricted use, distribution, and reproduction in any medium, provided the original work is properly cited.

Journal of Health, Sports, \& Kinesiology | ISSN 2692-9864 (Online) \& ISSN 2767-5874 (Print) | www.johsk.com 


\title{
10 ORIGINAL RESEARCH
}

\section{Anthropometric Somatotypes in Youth in a Rural School District in Kansas}

\author{
Skye Choi \& Young Sub Kwon
}

Humboldt State University, Arcata, CA, USA

Keywords: somatotypes, youth, childhood obesity

\begin{abstract}
Introduction: According to a report by the Trust for America's Health and the Robert Wood Johnson Foundation, the state of Kansas has the 47th highest rate of childhood obesity at $10.6 \%$ for children ages 10-17. It ranks the 22nd highest for obesity in high school students at $15.1 \%$ and 12 th highest for obesity in adults at $35.2 \%$.

Purpose: The purpose of the study was to verify somatotypical characteristics of K-12 students within a rural school district in the state of Kansas to chart and analyze how each grade level compares to one another and categorize children into one of three different anthropometric somatotypes.

Methods: The somatotypical values of 345 students were obtained via Heath-Carter's method for anthropometric measurements. Children were grouped into four different grade groups: $K-3(n=91), 4-$ $6(n=84), 7-9(n=88)$, and 10-12 ( $n=82)$, respectively. Data were taken to classify students in each grade group in one of the three following categories: endomorphic, mesomorphic, or ectomorphic. The Anthropometrics Standardization Reference Manual and the International Biological Program references were pursued anthropometric measurements. Measurements used to conduct somatotypical calculations include weight and height measurements, as well as skinfolds of the triceps, the subscapular, the supraspinale, and the calf. Other measurements include the circumferences of the biceps, the humerus bicondylar, the femoral bicondylar, and the calf. For statistical analysis, the STATISTICA version 7.1 software was used for statistical evaluation and ANOVA analyses. When a significant F-ratio was obtained, a Tukey's Honestly Significant Difference test was performed. Statistical significance was accepted at $p<0.05$. All data are presented as the mean \pm SD.
\end{abstract}

Results: Endomorphic scores of children in grades 10-12 (6.2 \pm 2.0$)$, 7-9 (6.2 \pm 2.7$)$, and 4-6 (5.9 \pm 2.7$)$ were higher than for those in K-3 $(3.6 \pm 1.9 ; p<0.05)$. Mesomorphic scores of children in grades K-3 (4.5 $\pm 1.2)$ were higher than for those in $7-9(3.1 \pm 1.3 ; p<0.05)$ and 10-12 (3.3 $\pm 1.6 ; p<0.05)$, and mesomorphic scores of children in grades 4-6 (4.5 \pm 4.5$)$ was higher than for those in 7-9 (3.1 $\pm 1.3 ; p<$ $0.05)$. Ectomorphic scores of children in grades $4-6(2.8 \pm 1.5), 7-9(3.0 \pm 1.5)$ were higher than for those

Journal of Health, Sports, \& Kinesiology | ISSN 2692-9864 (Online) \& ISSN 2767-5874 (Print)| www.johsk.com 
in K-3 $(2.2 \pm 1.2 ; p<0.05)$, and ectomorphic scores of children in 7-9 $(3.0 \pm 1.8 ; p<0.05)$ was higher than those in 10-12 $(2.3 \pm 1.2 ; p<0.05)$.

Conclusion: Significant differences were found between other grade groups. The comparison of the different anthropometric somatotypes of the four different grade groups shows that as children get older, the more endomorphic their physiques seem to be. From these results, it is apparent that the K3 grade group is considered ideal compared to other grade groups, as it has the lowest endomorphic score and a relatively higher mesomorphic score. Considering this finding, this school district could consider implementing physical activity and wellness education programs as early as the third grade. Generally, an earlier implementation of physical activity and wellness education programs could help to decrease the childhood obesity rate.

Discussion: General limitations of this study include that the data is not categorized by age nor sex, but rather generalized as a grade group. Other limitations could include the rural location of the school district, as rural areas tend to have a lower socioeconomic groups and limited access to foods, especially when compared to areas of higher populations and socioeconomic statuses. Areas of lower socioeconomic status may have food deserts, which would affect the diets of the local children, further affecting levels of obesity. Future studies should consider looking at multiple, intersectional factors such as age, sex, levels of physical activity, race/ethnicity, socioeconomic class, and levels of health education received.

\section{Author Information}

Skye Choi, https://orcid.org/0000-0002-4207-0651

Department of Kinesiology and Recreation Administration, Humboldt State University, Arcata, California, USA.

Email: skycblve@hotmail.com

Young Sub Kwon, Ph.D., https://orcid.org/0000-0001-8920-2757

Associate Professor, Department of Kinesiology and Recreation Administration, Humboldt State University, Arcata, California, USA.

Email: ysk15@humboldt.edu

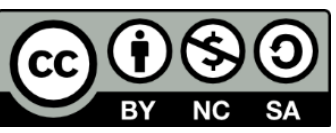

(C) 2021. This work is licensed under a CC BY-NC-SA 4.0 International license.

This is an open access article distributed under the Creative Commons Attribution License which permits unrestricted use, distribution, and reproduction in any medium, provided the original work is properly cited. 


\title{
11 ORIGINAL RESEARCH
}

\section{Leadership Traits and Self-Esteem of Selected Martial Arts Practitioners in the University of Santo Tomas}

\author{
Stewen R. Jain, Jan Michael Ezekiel E. Jaucian, \\ Zachary T. Huang, Michael David T. Lopez \\ University of Santo Tomas, Philippines
}

Keywords: leadership traits, self-esteem, martial arts, college students

\begin{abstract}
Introduction: Martial arts come in different forms around the world. Each martial arts discipline strives for mastery of both the physical and the psychological. People decide to join these disciplines to enhance their self-defense capabilities, cater to their interests, and pursue their passions. However, some people have a distorted mindset of martial arts because of how it is portrayed in media (Stickney, 2005). Quite a lot even perceive martial arts as promoting violence (Renata et al., 2016). Despite these negative perceptions, several studies emphasize the fact that engagement in martial arts had a positive effect on leadership skills, self-esteem, and confidence (Richman \& Rehberg, 1986).

Purpose: This study aims to determine the specific leadership traits (idealized influence, inspirational motivation, intellectual stimulation, individualized consideration, contingent reward, management by exception, and laissez-faire) and the levels of self-esteem of selected collegiate martial arts practitioners in the University of Santo Tomas. The study also intends to determine if there is a significant relationship between these two variables (specific leadership traits and self-esteem).

Methods: This quantitative study used the snowball method to recruit participants. There were a total of 60 UST college students who participated in this study, 46 of them are males and 14 are females. They come from different martial arts disciplines such as arnis, judo, karate, muay thai, and taekwondo. The instruments used in this study were the Self-Esteem Inventory (SEl; Coopersmith, 1967) and the Multifactor Leadership Questionnaire (MLQ; Avolio \& Bass, 2000). The SEI was designed to measure adolescents' and adults' attitudes or behavior towards the self in a variety of areas such as (family, peers, school, and general social activities). The MLQ aims to assess and determine the full range of leadership traits of the participant. The relationship between leadership traits and self-esteem was determined by using the Pearson correlational method.
\end{abstract}

Journal of Health, Sports, \& Kinesiology | ISSN 2692-9864 (Online) \& ISSN 2767-5874 (Print)| www.johsk.com 
Results: For leadership traits, the participants generally exhibited high levels in terms of the individualized consideration and management by exception subscales while the rest of the subscales reflected moderate levels. For self-esteem, the mean scores for the respondents generally demonstrated high levels of self-esteem $(M=74.45)$. For the relationship between the leadership traits and self-esteem, no significant relationship between these two variables were found, in any of the leadership trait subscales.

Conclusion: Based on the findings generated in this study, the following conclusions can be made (1) UST collegiate martial arts practitioners put high value into individualized consideration and management by exception when it comes to their leadership traits, (2) UST collegiate martial arts practitioners generally have high levels of self-esteem, and (3) there is no significant relationship between the leadership traits and the self-esteem of UST collegiate martial arts practitioners.

Discussion: The results of the study showed that there is no significant relationship between the participants' leadership traits and levels of self-esteem. This may be attributable to several factors such as the age of the participants, their years of engagement in their martial arts disciplines, and the research instruments that were used. Modifying these factors can be good starting points for future research on the topic, which is highly recommended as this can definitely benefit the collegiate martial arts students and their coaches, researchers, physical education teachers, and those involved in sport administration and governance.

\section{Author Information}

Stewen R. Jain, https://orcid.org/0000-0003-0042-7330

Institute of Physical Education and Athletics, University of Santo Tomas, Manila, Philippines. Email: rajuredashni97@gmail.com

Jan Michael Ezekiel E. Jaucian, https://orcid.org/0000-0001-5569-3922

Institute of Physical Education and Athletics, University of Santo Tomas, Manila, Philippines. Email: janmichael.jaucian.ipea@ust.edu.ph

Zachary T. Huang, https://orcid.org/0000-0002-2265-4481

Institute of Physical Education and Athletics, University of Santo Tomas, Manila, Philippines. Email: zacharylance.huang.ipea@ust.edu.ph

Michael David T. Lopez, https://orcid.org/0000-0002-5560-5942

Institute of Physical Education and Athletics, University of Santo Tomas, Manila, Philippines.

Email: $\underline{\text { mtlopez@ust.edu.ph }}$

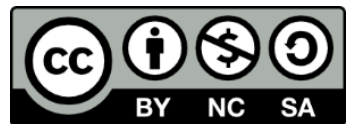

(C) 2021. This work is licensed under a CC BY-NC-SA 4.0 International license.

This is an open access article distributed under the Creative Commons Attribution License which permits unrestricted use, distribution, and reproduction in any medium, provided the original work is properly cited. 


\title{
12 ORIGINAL RESEARCH
}

\section{A Case Study: Teaching Materials Developments, Practices, and Applications of Using QR codes in Physical Education classes}

\author{
Boung Jin Kang \\ Elizabeth City State University, NC, USA
}

Keywords: QR codes, teaching materials, learning interest

\begin{abstract}
Introduction: QR codes can contain information such as lecture contents, URL links, assignments, or just about any Physical Education teaching method class information that can be embedded in a two-dimensional barcode. This encoded data can be decoded by scanning the barcode with a mobile device that is equipped with a camera and $Q R$ reader software.

Purpose: The purpose of this study was to investigate the developments, practices, and applications of pre-service physical education teachers about the use of QR codes in the teaching materials that they prepared in the teaching method course.

Methods: A total of 73 (22 female, 51 male) pre-service physical education teachers participated in this study in the Fall semester of the 2020-2021 academic year. For this case study, the qualitative data were collected through class observations, peer reviews, and formal-informal interviews. The data were analyzed by open-ended items (e.g., class observations and informal interviews), and formal interview data were analyzed using the constant comparative method.

Results: The study found the perspectives of prospective physical education teachers on the use of QR codes in the teaching materials they developed, in the learning process, its motivation, engagement, and effects of QR codes on the materials developments, practices, and applications. Specifically, first, it could improve all students' learning interests, motivation, and involvement. Second, technology can allow students to save class management and learning time for the activity class. The last, the knowledge of physical education technology applications can be expanded during the lecture.
\end{abstract}

Journal of Health, Sports, \& Kinesiology | ISSN 2692-9864 (Online) \& ISSN 2767-5874 (Print) | www.johsk.com 
| 2021 | Volume 2 | Issue 3(S) | The Journal of Health, Sports, and Kinesiology |

Conclusion/Discussion: The future suggestions of the technology in PE class are as follows. First, support for more technical systems for functional problems should be provided. Second, it is necessary to develop technologies associated with the PE curriculum. Third, educational content should be developed to cultivate cooperation and cohesion through technology. Fourth, the perception of PE class and technology will have to change. Finally, all pre-service PE teachers stated that QR codes could be used in game-based learning, gym instructions, class assessment, assignments, classroom boards, and school corridor at the end of the semester. The pre-service PE teachers listed the advantages of $Q R$ codes as short preparation time, easy preparation, portability, updateable, time saving, preventing paper wastage, and providing direct access to targeted information. The prospective PE teachers pointed out the disadvantages of QR codes as Internet connection problems, requiring tablets, smartphones, or smart devices and QR code preparation in paid applications.

\section{Author Information}

Boung Jin Kang, Ph.D., https://orcid.org/0000-0002-7637-1612

Associate Professor, Department of Health and Human Studies, Elizabeth City State University, North Carolina, USA. Email: $\underline{\text { bkang@ecsu.edu }}$ 


\title{
13 ORIGINAL RESEARCH
}

\section{Prospective Association of Handgrip Strength with Medical Utilization and the Risk of Hospitalization in Korean Adults}

\author{
Ju Seong Lee1, Young Sub Kwon'1,2, Nam Hun Kim ', Joo Eun Park', \\ Sin-Beom Kang ', Jae-Yong Jang ${ }^{1}$, \& Junghoon Kim ${ }^{1}$ \\ I Korea Maritime and Ocean University, Busan, South Korea \\ 2 Humboldt State University, Arcata, California, USA
}

Keywords: handgrip strength, hospitalization, sarcopenia

\begin{abstract}
Introduction: Age-related loss of skeletal muscle mass (sarcopenia) and/or strength may contribute to an increased risk of chronic disease and disabilities. To diagnose sarcopenia, handgrip strength has been evaluated as a decreased muscular function. In previous studies, reported that decreased handgrip strength was associated with increased risk of chronic disease, mortality, and decreased cognitive function. Nevertheless, the relationship between handgrip strength and health conditions such as medical use or hospitalization is unknown.

Purpose: This study aimed to investigate the prospective association of handgrip strength levels, medical utilization, and hospitalization in the general Korean adults aged $\geq 45$ years in baseline.

Methods: In this study, we selected 10,254 adults aged $\geq 45$ years from Korean Longitudinal Study of Ageing from 2006 to 2018. After excluded participants had missing data, all 9,228 were finally included in the study population. Handgrip strength was divided into quartiles by sex-specific cutoff point. All of the information for days for the use of medical care were obtained from medical receipts. The medical report was examined every 2 years from baseline (2006) to the end of follow-up in 2018. We calculated the utilization of outpatient and hospitalization medical care (days/years). We also generated binary variables $(0$ or 1$)$ for hospitalization defined as those who were hospitalized at least once a year. To examine the prospective effect of handgrip strength on outpatient use and hospitalization days, we used mixed effects models for repeated measured analysis. Furthermore, cox proportional hazard models were used to predict the risk of hospitalization from handgrip strength levels.
\end{abstract}

Results: Compared the highest handgrip strength groups, the group of lowest handgrip strength showed a significant higher hazard ratio (HR) of hospitalization in all models (HR: 1.22; $95 \%$ Cl: 1.11 1.34) after adjusted for covariates. We also found the longitudinal association of handgrip strength

Journal of Health, Sports, \& Kinesiology | ISSN 2692-9864 (Online) \& ISSN 2767-5874 (Print) | www.johsk.com 
levels and the use of hospitalization and outpatient medical care by using mixed-effects modes with time-dependent interaction. The estimated days of outpatient medical use in lower handgrip strength group (mean, 9.26 days/years; 95\% Cl: 6.97-11.55) was 3.7-times greater than the higher handgrip strength group (mean, 2.47 days/years; $95 \% \mathrm{Cl}$ : 0.64-4.31).

Discussion: We found that decreased handgrip strength levels contribute to increased risk of hospitalization during 12-years follow-up in the Korean population. Moreover, days of outpatient medical use were higher compared with the higher handgrip strength group. It is intuitive to suggest that having low muscle mass and strength may lead to more functional limitations and more metabolic diseases, which may be caused by geriatric conditions. In fact, loss of skeletal muscle mass has been reported to be related to increased geriatric conditions (i.e., falls, delirium, malnutrition, and disability), contributing to chronic diseases. Although handgrip strength reflects the muscle strength of the whole body, there may be limits to its ability as an indicator of muscular strength. Moreover, we used only handgrip strength as the parameter for sarcopenia and did not use muscle mass. Further studies involving muscle mass may be needed. However, we could infer a cause-and-effect association between handgrip strength and medical health care because of longitudinal study design among middle and older aged of the large general population.

Conclusion: Lower handgrip strength was associated with increased risk of hospitalization and use of medical care in Korea. This study highlights the maintaining of muscular strength may be essential to reduce of risk for hospitalization and medical use by preventing chronic disease.

Acknowledgment: This work was supported by the "Leaders in INdustry-university Cooperation +" Project grant funded by the Ministry of Education and National Research Foundation of Korea (Grant Number: 2021EG055010117) and the Ministry of Oceans and Fisheries (Grant Number: KOFPI2021410C10-2125-0101)

Conflict of Interest: The authors declare no conflict of interest.

\section{Author Information}

Ju Seong Lee, https://orcid.org/0000-0001-5413-3747

Department of Sports Medicine, Graduated School of Sports Convergence, Korea Maritime and Ocean University, Busan, Republic of Korea. Email: wntjdaos2@gmail.com

Young Sub Kwon, Ph.D., https://orcid.org/0000-0001-8920-2757

Associate Professor, Department of Kinesiology and Recreation Administration, Humboldt State University, Arcata, California, USA; Department of Sports Medicine, Graduated School of Sports Convergence, Korea Maritime and Ocean University, Busan, Republic of Korea.

Email: ysk15@humboldt.edu

Nam Hun Kim, https://orcid.org/0000-0002-3821-7999

Department of Sports Medicine, Graduated School of Sports Convergence, Korea Maritime and Ocean University, Busan, Republic of Korea.

Email: skagns26506183@gmail.com

Journal of Health, Sports, \& Kinesiology | ISSN 2692-9864 (Online) \& ISSN 2767-5874 (Print)| www.johsk.com 
| 2021 | Volume 2 | Issue 3(S) | The Journal of Health, Sports, and Kinesiology |

Joo Eun Park, https://orcid.org/0000-0002-0155-9573

Department of Sports Medicine, Graduated School of Sports Convergence, Korea Maritime and Ocean University, Busan, Republic of Korea.

Email: pje9444@gmail.com

Sin-Beom Kang, Ph.D., https://orcid.org/0000-0003-3528-494X

Professor, Department of Sports Medicine, Graduated School of Sports Convergence and Department of Ocean Sports Science, Korea Maritime and Ocean University, Busan, Republic of Korea.

Email: kangsb@kmou.ac.kr

Jae-Yong Jang, Ph.D., https://orcid.org/0000-0003-4703-9685

Associate Professor, Department of Ocean Sports Science, Korea Maritime and Ocean University, Busan, Republic of Korea.

Email: marinboy707@hanmail.net

Junghoon Kim, Ph.D., https://orcid.org/0000-0003-4802-010X

Assistant Professor, Department of Sports Medicine, Graduated School of Sports Convergence and Department of Ocean Sports Science, Korea Maritime and Ocean University, Busan, Republic of Korea. Email: junghoonkim@kmou.ac.kr

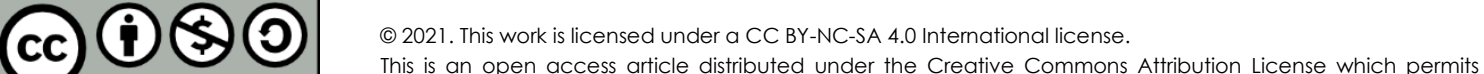
unrestricted use, distribution, and reproduction in any medium, provided the original work is properly cited.
} 
| 2021 | Volume 2 | Issue 3(S) | The Journal of Health, Sports, and Kinesiology |

Journal of Health, Sports, and Kinesiology, 2021, 2(3), 37-38. https://doi.org/10.47544/johsk.202 1.2.3

() 2020-2021 by the International Organization for Health, Sports, \& Kinesiology

\title{
14 ORIGINAL RESEARCH
}

\section{Resting Heart Rate is a Prognostic Factor for Mortality in Patients with Colorectal Cancer}

\author{
Wonhee Cho', Justin Y. Jeon², \& Joon Young Kim¹ \\ ' Syracuse University, New York, USA \\ 2 Yonsei University, Seoul, South Korea
}

Keywords: resting heart rate, colorectal cancer, prognosis

\begin{abstract}
INTRODUCTION: Colorectal cancer is a leading cause of death in the world and US. Although the colorectal surgery reduces overall mortality rate, there are individual variations (exhibiting $50-90 \%$ of 5 -year survival rate after the surgery). In this regard, an accurate prediction (using preoperative characteristics) of mortality after the sugery in colorectal cancer patients has been highlighted. One such modifiable prognostic indicator of mortality is resting heart rate (RHR). To date, elevated RHR was reported to be highly associated with increased mortality rate in the general population and patients with various cancers such as lung and breast cancer. However, there is lack of evidence to support whether RHR at the day of surgery could indicate mortality in patients with colorectal cancer over time.
\end{abstract}

PURPOSE: We aimed to see if RHR is a significant predictor of all-cause and cancer-specific mortality in patients with colorectal cancer who underwent colon or rectal cancer surgery.

METHODS: Baseline data from the Severance Hospital Colorectal Cancer Registry (from December 2010 through December 2015) was utilized for the present retrospective study with ongoing follow-up screening ( $~ 10$ years), including a total of 3,547 colorectal cancer patients with stage I-III who underwent colon or rectal cancer surgery. The Registry Database includes physical examination (weight, height, BMl, blood pressure), disease history, date and types of surgery, location of the primary tumor, TNM (tumor, node, metastasis) stage, histology, the number of lymph node, ASA score, the regimen of adjuvant therapy, alcohol and smoking history, and follow-up outcomes related to colorectal cancer. All of the aforementioned varilables were used as covariates for the analysis. RHR (beats per minute; bpm) data were collected on the day of surgery after $>5$ min of resting in a supine position. The primary outcomes were (1) all-cause mortality calculated from the date of colorectal cancer surgery to the date of the death from any cause, and (2) colorectal cancer-specific mortality collected from the medical chart review. Analysis of variance and $x^{2}$ test were used to compare the

Journal of Health, Sports, \& Kinesiology | ISSN 2692-9864 (Online) \& ISSN 2767-5874 (Print) | www.johsk.com 
patients' baseline characteristics. Multivariable Cox-proportional hazards models were conducted to estimate hazard ratios (HRs) of RHR with $95 \%$ confidence interval (CI) for all-cause mortality and colorectal cancer-specific mortality. All statistical analyses were conducted by SAS 9.4 (SAS, NC, USA) with a level of significance set at $p$ value $<0.05$.

RESULTS: A total of 3,547 colorectal cancer patients was divided into 5 groups based on their RHR:

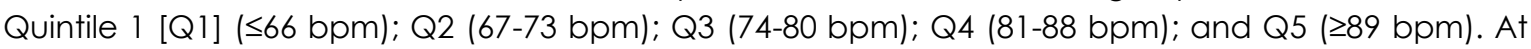
baseline, Q5 had more disease histories, number of positive lymph node, poor histology, and higher TNM stage among the groups. Over the 10-years follow-up period, 320 death cases from all-cause and 202 death cases from colorectal cancer-specific were reported. There was a significant and prospective relationship between RHR and all-cause mortality in unadjusted and adjusted models: (unadjusted HR Q5 vs. Q1: 4.46, 95 \% Cl: 2.26-6.96) and (adjusted HR Q5 vs. Q1: 4.26, 95\% Cl: 2.68-6.78). Similarly, RHR is significantly predictive of colorectal cancer-specific mortality: (unadjusted HR Q5 vs. Q1: $4.46,95 \% \mathrm{Cl}: 2.26-6.96$ ) and (adjusted HR Q5 vs. Q1: 4.26, $95 \% \mathrm{Cl}: 2.68-6.78$ ). It is notable that HRs $(95 \% \mathrm{CI})$ for all-cause mortality and colorectal cancer-specific mortality with RHR per 10 bpm increase was $1.46(1.34-1.59)$ and $1.49(1.34-1.65)$, respectively.

CONCLUSION: Increased RHR was associated with all-cause and colorectal cancer-specific mortality in patients with colorectal cancer who underwent the surgery. It could be postulated that elevated RHR reflects an increased sympathetic nerve system, which might affect the beta-adrenergic signaling that contribute to cancer progression (i.e., worsen mortality even after the surgery). In line with this theory, our data suggest that baseline RHR of the colorectal cancer patients who will have their surgery can be a clinically relevant prognostic indicator of future mortality. Further research should be warranted to identify efficient strategies to modify RHR prior to the surgery to improve overall survival in colorectal cancer patients.

\section{Author Information}

Wonhee Cho, https://orcid.org/0000-0003-3850-1495

Department of Exercise Science, David B. Falk College of Sport and Human Dynamics, Syracuse University, Syracuse, NY, USA.

Email:wcho02@syr.edu

Justin Y. Jeon

Department of Sport Industries, Yonsei University, Seoul, South Korea.

Joon Young Kim, Ph.D., https://orcid.org/0000-0003-0448-1684

Assistant Professor, Department of Exercise Science, David B. Falk College of Sport and Human Dynamics, Syracuse University, Syracuse, NY, USA.

Email: ikim291@syr.edu

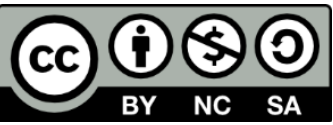

(C) 2021. This work is licensed under a CC BY-NC-SA 4.0 International license.

This is an open access article distributed under the Creative Commons Attribution License which permits unrestricted use, distribution, and reproduction in any medium, provided the original work is properly cited.

Journal of Health, Sports, \& Kinesiology | ISSN 2692-9864 (Online) \& ISSN 2767-5874 (Print) | www.johsk.com 


\title{
15 ORIGINAL RESEARCH
}

\section{The combination of novel biomarkers identifies individuals of heighten risk for type 2 diabetes}

\author{
Jared Rosenberg' \& Joon Young Kim ${ }^{1,2}$ \\ ' Syracuse University, New York, USA \\ ${ }^{2}$ Arizona State University, AZ, USA
}

Keywords: oral glucose tolerance test, one-hour glucose concentration, glucose response curve

\section{ABSTRACT}

Introduction: In adults, both the glucose response curve (GRC) and one-hour glucose concentration (1HGC) during an oral glucose tolerance test (OGTT) have emerged as sensitive risk biomarkers for type 2 diabetes (T2D). Adults with monophasic response curve (MRC) compared with biphasic response curve (BRC) and $1 \mathrm{HGC} \geq 155 \mathrm{mg} / \mathrm{dL}$ (above155) compared with $1 \mathrm{HGC}<155 \mathrm{mg} / \mathrm{dL}$ (below155) exhibited worse insulin sensitivity and $\beta$-cell dysfunction, indicating a heigh risk for T2D. Although those two biomarkers are derived from the same test, a recent study in youth found that only $50 \%$ of individuals with above155 had MRC (Cree-Green, JES, 2018), signifying a unique pathophysiology involved in those two. Currently, it is unknown if simultaneous use of GRC and 1HGC as a risk identification tool can provide a clinical relevance. Therefore, the purpose of this study is to investigate whether the combination of GRC and $1 \mathrm{HGC}$ can differentiate pathophysiological risk factors for T2D in Latino adults.

Methods: A total of 434 Latino adults (age $37.0 \pm 9.5$ [SD] years; 269F/165M; body mass index: $29.8 \pm 5.6$ $\mathrm{kg} / \mathrm{m}^{2}$ ) underwent a 2-hour OGTT (75g dextrose) and venous blood samples were obtained at $-15,0$, $30,60,90$, and 120 minutes for the measurement of glucose and insulin concentrations. All participants were divided into 4 groups based on their GRC and 1 HG phenotypes: below155/biphasic (BB), above155/biphasic (AB), below155/monophasic (BM), and above155/monophasic (AM). Matsuda index (i.e., whole-body insulin sensitivity index) was calculated as 10,000/ $\sqrt{ }$ (ffasting glucose $x$ fasting insulin) $\times$ (mean OGTT glucose $x$ mean OGTT insulin)), insulinogenic index (IGI) as ( $\Delta$ Inso-30)/( $\Delta$ Gluo-30), and oral disposition index (ODI) as (Matsuda index $x$ IGI). One-way ANOVA with Bonferroni's post-hoc tests was used to examine differences in insulin sensitivity and $\beta$-cell function among 4 groups, significance was set at $\mathrm{p}<0.05$.

Results: There is a significant difference in Matsuda index among the four groups, with the two highest values observed in the group of BB and BM ( $6.3 \pm 3.6$ and 6.2 \pm 3.7 , respectively) compared with the

Journal of Health, Sports, \& Kinesiology | ISSN 2692-9864 (Online) \& ISSN 2767-5874 (Print) | www.johsk.com 
other two including $A B$ and $A M(3.4 \pm 2.0$ and $4.1 \pm 3.4$, respectively) (ANOVA $p<0.001)$. Additionally, the similar pattern of the highest oDI being observed in the BB and BM groups $(8.5 \pm 5.6$ and $8.1 \pm 5.0$, respectively) compared with the other two of $A B$ and $A M(3.2 \pm 2.1$ and $3.1 \pm 2.0$, respectively) was found (ANOVA $p<0.001)$. However, there was no difference in IGI between the groups $(p=0.8)$.

Conclusion: The combination of the GRC and IHGC was able to identify adults who were pathophysiological progressed toward the development of T2D, evidenced by consistent observations of the above155 (regardless of their GRC phenotypes) having severe insulin resistance (lower whole-body insulin sensitivity) and worse $\beta$-cell function (lower oDI). It is notable that decrement of $\mathrm{ODI}$ is the best metabolic predictor of future T2D in adults. Prospective studies should be warranted to examine whether the combination of the novel biomarkers, GRC and $1 \mathrm{HGC}$ of $155 \mathrm{mg} / \mathrm{dL}$, can predict future development of T2D.

Funding: National Institutes of Health/National Institute on Minority Health and Health Disparities (P20MD002316).

\section{Author Information}

Jared Rosenberg, https://orcid.org/0000-0002-1791-6790

Department of Exercise Science, David B. Falk College of Sport and Human Dynamics, Syracuse University, Syracuse, NY, USA.

Email: jarosenb@syr.edu

Joon Young Kim, Ph.D., https://orcid.org/0000-0003-0448-1684

Assistant Professor, Department of Exercise Science, David B. Falk College of Sport and Human Dynamics, Syracuse University, Syracuse, NY, USA; Center for Health Promotion and Disease Prevention, Arizona State University, AZ, USA.

Email: jkim291@syr.edu

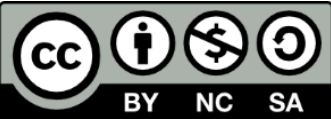

(c) 2021. This work is licensed under a CC BY-NC-SA 4.0 International license.

This is an open access article distributed under the Creative Commons Attribution License which permits unrestricted use, distribution, and reproduction in any medium, provided the original work is properly cited. 


\title{
16 ORIGINAL RESEARCH
}

\section{Moderate-Load Muscular Endurance Descending Resistance Training did not Improve 1RM in Resistance-Trained Men}

\author{
Hyun Min Kim1, Junghoon Kim', Min Hyuk Kwon', \\ Yun Seo Choi ${ }^{1}$, \& Young Sub Kwon ${ }^{1,2}$ \\ ' Korea Maritime and Ocean University, Busan, South Korea \\ 2 Humboldt State University, Arcata, California, USA
}

Keywords: constant resistance, drop sets, repetition maximum

\begin{abstract}
Introduction: We previously reported that a heavier 1 repetition maximum (RM) and a greater training volume response were attained using the descending resistance (DR) training method compared to a constant resistance (CR) training method in both males and females after a 4 -week hypertrophy training program.

Purpose: When a resistance-trained individual performs multiple sets with a short rest period, such as 1 minute and 30 seconds between sets, the resistance must be reduced to maintain the same number of repetitions until fatigue. Otherwise, the number of repetitions will be reduced if the constant resistance is used. There is no standard method for optimizing load to maximize training volume when using multiple sets with short rest periods. In this study, we hypothesized that a heavier IRM would be produced using a 4-week moderate-load endurance training method where resistance is decreased with each set (DR) compared to a 4-week moderate-load CR method. To develop DR sets, we used the subject's fatigue ratio (using an individualized regression equation) from a CR protocol where the number of repetitions declines with each set.

Methods: Twelve resistance-trained male subjects (mean \pm SD, age $=28 \pm 6$ yr., height $=175 \pm 4 \mathrm{~cm}$, body mass $=74 \pm 7 \mathrm{~kg}, 1 \mathrm{RM}=121 \pm 9 \mathrm{~kg}$, weight training experience $=6 \pm 2 \mathrm{yr}$, and strength ratio $(1 \mathrm{RM} / \mathrm{body}$ mass) $=1.63 \pm 0.1$ ) completed pre and post 1RM tests. During these pre and post 1RM tests, moderate muscular endurance intensities equivalent to $65 \%$ IRM CR and DR bench press exercise trainings were performed in counterbalanced order. Data were analyzed using a one-way repeated-measures ANOVA.
\end{abstract}

Journal of Health, Sports, \& Kinesiology | ISSN 2692-9864 (Online) \& ISSN 2767-5874 (Print) | www.johsk.com 
Results: There was a significant increase with 1 RM $(p<0.05)$ when subjects trained with CR (Pre:

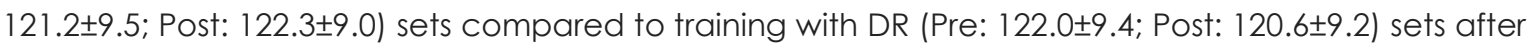
a 4 -week endurance training period for each condition.

Conclusion: In resistance-trained men, a lighter IRM was attained using a 4-week moderate-load muscular endurance training on a DR training method than a CR training method. This suggests that $C R$ training may be more effective than DR training for maintaining strength after a 4-week endurance training program.

Discussion: We obtained a greater 1RM after a 4-week hypertrophy training program at $80 \%$ and $75 \%$ of 1 RM using a DR training method than a CR training method in resistance-trained men in the previous study. However, we found a lighter IRM after a 4-week endurance training program using a 65\% 1RM DR training method than a $65 \% 1$ RM CR training method in this study. The effects of the training method might be depending on which \% of 1 RM load of the training program was used. A 4-week $65 \%$ of 1 RM load endurance training program using a DR training method was not sufficient to elicit improvement in strength. Therefore, further studies are needed to find an optimal load muscular endurance DR training method on muscle strength in resistance-trained men.

\section{Author Information}

Hyun Min Kim, https://orcid.org/0000-0003-0865-0211

Department of Sports Medicine, Graduated School of Sports Convergence, Korea Maritime and Ocean University, Busan, Republic of Korea. Email: khm58322@gmail.com

Junghoon Kim, Ph.D., https://orcid.org/0000-0003-4802-010X Email: junghoonkim@kmou.ac.kr Assistant Professor, Department of Sports Medicine, Graduated School of Sports Convergence and Department of Ocean Sports Science, Korea Maritime and Ocean University, Busan, Republic of Korea.

Min Hyuk Kwon, https://orcid.org/0000-0003-1713-2539

Department of Sports Medicine, Graduated School of Sports Convergence, Korea Maritime and Ocean University, Busan, Republic of Korea. Email: domodal@naver.com

Yun Seo Choi, https://orcid.org/0000-0002-3821-7999

Department of Sports Medicine, Graduated School of Sports Convergence, Korea Maritime and Ocean University, Busan, Republic of Korea. Email: dbsti69@gmail.com

Young Sub Kwon, Ph.D., https://orcid.org/0000-0001-8920-2757 Email: ysk15@humboldt.edu Associate Professor, Department of Kinesiology and Recreation Administration, Humboldt State University, Arcata, California, USA; Department of Sports Medicine, Graduated School of Sports Convergence, Korea Maritime and Ocean University, Busan, Republic of Korea.

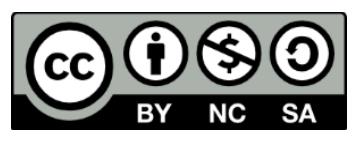

(c) 2021. This work is licensed under a CC BY-NC-SA 4.0 International license.

This is an open access article distributed under the Creative Commons Attribution License which permits unrestricted use, distribution, and reproduction in any medium, provided the original work is properly cited.

Journal of Health, Sports, \& Kinesiology | ISSN 2692-9864 (Online) \& ISSN 2767-5874 (Print) | www.johsk.com 


\title{
17 UNDERGRADUATE RESEARCH CRITIQUE
}

\section{Research Critique on "The Effects of Spirulina Under High-Intensity Interval Training on Levels of Nesfatin-1, Omentin-1, and Lipid Profiles in Overweight and Obese Females: A Randomized, Controlled, Single-Blind Trial"}

\author{
Alyssa H. Shin', Ohjun Kwon², Seoyoung Moon ${ }^{3}$, \& Young Sub Kwon ${ }^{4}$ \\ I St. Bernard's Academy, Eureka, CA, USA \\ 2 LOL technology, Seoul, South Korea \\ 3 Inha University, South Korea \\ 4 Humboldt State University, Arcata, California, USA
}

Keywords: spirulina, high-intensity interval training, overweight and obese females, nesfatin-1, omentin-1, Lipid profiles

\begin{abstract}
Introduction: Obesity is a significant issue considering health. When confronted with the problem, people suppose exercise to be the primary solution. However, bioactive supplementation is also an attractive way to manage obesity and physiological conditions. These days, the combinatorial effects of both methods highlight a powerful direction for improving health. Spirulina, a photosynthetic cyanobacteria, is a GRAS-level superfood in respect to its antioxidative and anti-inflammatory characteristics and high concentration of protein and minerals. For this reason, researchers have performed studies about the effect of spirulina supplements under several exercise conditions worldwide.

Purpose/Objective: Overweight and obese females were examined for their levels of nesfatin-1, omentin-1, and lipid profiles under spirulina supplementation and high-intensity interval training (HIIT).

Methods: The research follows a randomized, quasi-experimental controlled, and single-blinded with a pre- and post-test design. Twenty overweight and obese women (body mass index $=29.32 \pm 3.01$ $\mathrm{kg} / \mathrm{m}^{2}$, age $=21.55 \pm 1.76$ years) were randomly allocated into HIIT + spirulina $(n=10)$ and HIIT + placebo $(n=10)$. Running an anaerobic sprint test for HIIT protocol consists of six intervals at $35 \mathrm{~m}$ maximal speed runs, with a rest of $10 \mathrm{~s}$ in each trial ( 3 times/week, 4 weeks). The HIIT + spirulina group took $500 \mathrm{mg}$ of spirulina pills twice daily for 4 weeks.
\end{abstract}

Journal of Health, Sports, \& Kinesiology | ISSN 2692-9864 (Online) \& ISSN 2767-5874 (Print) | www.johsk.com 
Results: The HIIT+spirulina group showed significantly increased serum levels of nesfatin-1 $(p<0.0001)$ but the HIIT + placebo $(p=0.61)$ group did not. Serum levels of nesfatin-1 were significantly different between the two groups $(p=0.04)$. In the case of omentin-1, its serum levels increased in both groups $(p<0.05)$, and there was no significant difference between the groups $(p=0.49)$. In addition, no interand intra-group differences were significantly observed in total cholesterol levels, triglycerides, lowdensity lipoprotein cholesterol, and high-density lipoprotein cholesterol between the groups $(p>0.05)$.

Conclusion: Overweight and obese females expressed increased levels of both nesfatin-1 and omentin-1 but not for the level of lipid profiles under spirulina supplementation and HIIT.

Critique: The primary significance of this work is that HIIT+spirulina increased serum levels of nesfatin-I in overweight and obese females, not affecting lipid profiles. However, further discussion is needed on the design and results of the study since the results are unclear. Also, the authors acknowledge limitations of this study, even though the results are well worthwhile, due to very few reports of the combinatorial effect of spirulina and exercise on women. There are some limitations found in the work. As the authors acknowledge, a short experimental period, a low dose of spirulina pills, and an inadequate number of participants can be limitations. However, more importantly, the study should have had four experimental groups, adding HIIT(X)+spirulina, HIIT(O)+placebo to the pre-existing two groups. Having more groups would distinguish the effect of each factor from the overall response, as shown in others. Also, the authors might have expected dramatic variations of biomarkers in their design. However, the given ambiguous results except for nesfatin-1 might have led them to discuss experimental limitations instead of the results. The authors should have focused on the physiological meaning of the results in their design because the variations can be meaningful in women's health. Furthermore, this study verified that spirulina supplement influences biomarkers of this study for women despite some limitations. As the authors acknowledge, spirulina dose and training period are the main factors. Therefore, a statistical experimental design would be helpful to identify the sensitivity of each factor on responses. Fractional factorial designs are feasible because they are adequate to analyze results with limited participants.

\section{Funding/Acknowledgement}

This work was supported by a private research grant from LOL technology.

\section{Author Information}

Alyssa H. Shin, https://orcid.org/0000-0001-8505-9252

St. Bernard's Academy, Eureka, CA, USA.

Email:shina23@saintbernards.us

Ohjun Kwon, https://orcid.org/0000-0003-2869-2742

LOL technology, Seoul, South Korea.

Email: charleskwon@loltechnology.com

Seoyoung Moon, https://orcid.org/0000-0001-7256-7004

Department of Economics, Inha University, South Korea.

Email: moonseoyoung0922@naver.com

Journal of Health, Sports, \& Kinesiology | ISSN 2692-9864 (Online) \& ISSN 2767-5874 (Print) | www.johsk.com 
| 2021 | Volume 2 | Issue 3(S) | The Journal of Health, Sports, and Kinesiology |

Young Sub Kwon, Ph.D., https://orcid.org/0000-0001-8920-2757

Associate Professor, Department of Kinesiology and Recreation Administration, Humboldt State University, Arcata, California, USA.

Email: ysk15@humboldt.edu

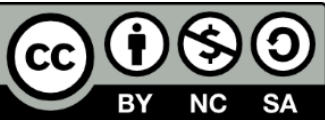

(C) 2021. This work is licensed under a CC BY-NC-SA 4.0 International license.

This is an open access article distributed under the Creative Commons Attribution License which permits

unrestricted use, distribution, and reproduction in any medium, provided the original work is properly cited. 


\title{
18 UNDERGRADUATE RESEARCH CRITIQUE
}

\section{Research Critique on "Footwear Insoles with Higher Frictional Properties Enhance Performance by Reducing In-Shoe Sliding During Rapid Changes of Direction"}

\author{
Sydney Williams \& MinHyuk Kwon \\ California State Polytechnic University, Pomona, CA, USA \\ Keywords: friction, insole, cutting
}

\begin{abstract}
Introduction: Many team sports require rapid changes in directions that include the whole body. The foot faces large shear forces when performing movements like cuts and turns. However, the frictional properties of footwear can counteract these forces and reduce the amount of time needed to change direction. The amount of grip provided by sports footwear can determine adaptations sports players use for optimal performance.

Purpose: The purpose of this study was to determine if insoles with increased mechanical friction enhance actual and perceived change of direction performance.

Methods: Fifteen healthy participants ( 11 men and 4 women, $25 \pm 4$ years) completed 5 trials of a side cut (at a 20-degree angle) and 5 trials of a complete turn (at a 180-degree angle) with $90 \%$ of their self-reported max speed. Data were collected using force plates (Kistler) and a six Oqus camera motion capture system (Qualysis). Coefficient of friction (COF) and ground reaction force (GRF) was analyzed in braking and propulsive phases in both movements. Biomechanical data were analyzed using Visual 3D software. repeated measures multivariate analysis of variance was performed to determine mechanical frictions between insole types.

Results: Participants perceived the same comfort level between insole types. For the side cut, there were no differences in COF $(p<.01)$, GRF $(p<.01)$, or contact time $(p<.01)$ during the braking and propulsive phases between insole types. For the turn, SI (Standard Insole) exhibited less mechanical friction [COF $(p<.01)$; GRF $(p<.01)$ ], leading to increased in-shoe sliding compared to TI (Training Insole). There were no differences in contact time $(p<.01)$ in either phase during the turn between insole types. Additionally, participants had to use more force to begin moving in the $\mathrm{Tl}$, resulting in greater static and dynamic COF.
\end{abstract}

Journal of Health, Sports, \& Kinesiology | ISSN 2692-9864 (Online) \& ISSN 2767-5874 (Print) | www.johsk.com 
Conclusion: The study found that the insole with enhanced mechanical friction (TI) increases both actual and perception of performance. These findings indicate that frictional properties in footwear insoles can enhance performance during changes in direction. The authors suggested that future developments in footwear and insoles should stress the importance of maintaining foot position for performance benefits.

Critique: The objective of the study was to determine if insoles with increased mechanical friction (TI) could enhance actual and perceived performance. The study evaluated COF and GRF. Findings suggest that TI decreases COF, GRF, and in-shoe sliding, thus keeping the foot in a better position during direction changes with a greater perception of performance. A notable strength of the study was the addition of a controlled variable for footwear. All participants were fitted with flexible shoes developed without midsole and outsole support. Having controlled footwear was vital as it allowed the study to highlight how the insole affected participant performance. It would be beneficial to the study to investigate rotational friction because the body has to create rotational friction to change direction. Therefore, analyzing how rotational friction changes between insole types during each movement can expand footwear and performance literature. Jumping would have also been a beneficial addition. Jumping is a fundamental skill in sports where gaining height or distance is an advantage. The addition of jumping to the study would highlight how the insole types effect braking and propulsive phases when moving vertically. COF, GRF, and rotational motion could be evaluated in the study while examining jump landing mechanics. Changes in deceleration mechanics based on insole type could also be evaluated When decelerating, the body has to absorb force then quickly create force to change directions. Analyzing braking and propulsive phases during this movement in each insole type could be beneficial to the study. Lastly, though the inclusion of a controlled variable for footwear in the study is advantageous, using footwear with standard features would aid in making the research translate to practice. Taking these suggestions into consideration would expand the literature on footwear for future studies.

\section{Author Information}

\section{Sydney Williams}

Department of Kinesiology and Health Promotion, California State Polytechnic University, Pomona, CA, USA.

Email: sydneyw@cpp.edu

MinHyuk Kwon, Ph.D., https://orcid.org/0000-0002-7008-7995

Assistant Professor, Department of Kinesiology and Health Promotion, California State Polytechnic University, Pomona, CA, USA.

Email: minhyukkwon@cpp.edu

(c)(®)(

(c) 2021. This work is licensed under a CC BY-NC-SA 4.0 International license.

This is an open access article distributed under the Creative Commons Attribution License which permits unrestricted use, distribution, and reproduction in any medium, provided the original work is properly cited. 


\title{
19 UNDERGRADUATE RESEARCH CRITIQUE
}

\section{Research Critique on "Influence of Sports Flooring and Shoes on Impact Forces and Performance During Jump Tasks"}

\author{
Shannon Sung-Jin Kim \& MinHyuk Kwon \\ California State Polytechnic University, Pomona, CA, USA
}

Keywords: jumping, sports flooring, shoes

\begin{abstract}
Introduction: Jumping serves an imperative role in increasing the performance of athletes of numerous sports, which also induces lower extremity injuries due to the impact force generated by the movement. It was evident that jumping on sports flooring with proper shoes could reduce the risk of injures. However, the interaction between them is biomechanically unclear.

Purpose: The purpose of this critiqued study was to identify the effectiveness of sports flooring and shoes when performing jump tasks.

Methods: Twenty-one healthy participants (Men, $26.8 \pm 5.7$ years) performed two types of jump tasks as follows; 1) four maximal jumps consecutively while maintaining strong ankle movements with full knee extensions (Ankle jump task). 2) two maximal countermovement jumps with knee flexion at approximately $90^{\circ}$ (Multi-jump task). The participants conducted both jump tasks on a combination of five sports flooring (SF) conditions [thickness: $7.5 \mathrm{~mm}$ (SF2), $11 \mathrm{~mm}$ (SF3), $14 \mathrm{~mm}$ (SF1), and $18 \mathrm{~mm}$ (SF4), no flooring (SFO)] and two types of shoes (non and cushioned shoes). Three trials were performed for each sports flooring and shoe combination (total of 10). The vertical ground reaction force (Fz), peak vertical ground reaction force (PVGRF), and vertical instantaneous loading rate (VILR) were recorded using two Arsalis 3D force plate systems. Jump performance was assessed by jump height and concentric work (Wcon). The analysis of variance test (ANOVA) was performed to determine the individual effectiveness of each shoe and flooring types on impact forces and jump performance during each jump task.
\end{abstract}

Results: Sports flooring was more effective in lowering VILR $(\mathrm{p}<0.001)$ compared to the PVGRF $(\mathrm{p}=$ $0.636)$ during ankle jump task. SF4 lowered VILR more than SFO ( $p=0.012)$ and SF1 ( $p=0.018)$ when non-cushioned shoes were worn. Compared to SFO, SF1, and SF2, VILR was also lower in SF4 when cushioned shoes were worn $(p<0.001)$ as well as compared to SF3 $(p=0.035)$. VILR was the lowest in

Journal of Health, Sports, \& Kinesiology | ISSN 2692-9864 (Online) \& ISSN 2767-5874 (Print) | www.johsk.com 
SF4 compared to SFO $(p<0.001)$ and SF2 $(p=0.004)$ during multi-jumps with non-cushioned shoes. No considerable differences on VILR were identified for all flooring types with cushioned shoes. Sports flooring did not affect PVGRF during multi-jumps. However, shoe types had a greater influence on both VILR and PVGRF. VILR was lower with cushioned shoes during both ankle jumps (95\%Cl: -943 to $607 \mathrm{~N} . \mathrm{s}^{-1} . \mathrm{kg}^{-1}, \omega^{2}=0.31$ ) and multi-jumps (95\%Cl: -979 to $\left.-627 \mathrm{~N} . \mathrm{s}^{-1} . \mathrm{kg}^{-1}, \omega^{2}=0.31\right)$. There was an increase in PVGRF during ankle jumps with cushioned shoes, while PVGRF was lower with noncushioned shoes during multi-jumps. Cushioned shoes also appeared to increase jump performance (higher jump height) during multi-jumps than non-cushioned shoes $(95 \% \mathrm{Cl}$ : 0.3 to $0.9 \mathrm{~cm}$; $\left.\omega^{2}=0.06\right)$

Conclusion: Sports flooring and shoes mostly influenced VILR. However, shoe types had more influence on impact force and jump performance variables, increasing jump height and peak ground reaction force. Cushioned shoes were found to be the most effective in lowering VILR for both jump tasks. As higher VILR is linked to sports-related injuries, cushioned shoes are the more preferred type of shoewear for reducing sports injuries.

Critique: The focus of the study was to determine the effectiveness of sports flooring and shoe types on impact force andjump performance. The research revealed that sports flooring and shoes lessen VILR and PVGRF. However, shoe types had a greaterinfluence on both impact force and jump performance-related variables. As multiple combinations of sports flooring and shoes were utilized, various sports environments were replicated. Therefore, the research can highlight the importance of creating sport-specific shoes that can effectively decrease VILR and improve jump performance. A limitation to the research is that only twenty-one male participants were studied. This could have contributed to the difficulty of indentifying the effectiveness of sports flooring and shoe types on jump height. A bigger sample size could have yielded values that show a higher presence of possible statistical significance between jump height and jump performance. In addition, the research may not be applicable to women athletes due to potential reasons such as women having less lowerbody strength and a lower center of mass. The research could have also produced more accurate results by having the participants perform a back arm swing while jumping. The participants could have been hindered from jumping maximally as their hands were positioned on their hips. Future studies should focus on testing sport-specific jump techniques to determine ideal sports shoes and sports flooring combinations that effectively reduce injuries and enhance jump performance.

\section{Author Information}

Shannon Sung-Jin Kim https://orcid.org/0000-0003-1686-7613 USA. Email: shan.sjkim@gmail.com Department of Kinesiology and Health Promotion, California State Polytechnic University, Pomona, CA,

MinHyuk Kwon, Ph.D. https://orcid.org/0000-0002-7008-7995 Email: minhyukkwon@cpp.edu Assistant Professor, Department of Kinesiology and Health Promotion, California State Polytechnic University, Pomona, CA, USA.

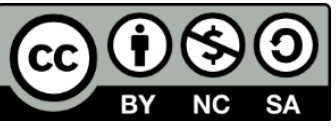

(C) 2021. This work is licensed under a CC BY-NC-SA 4.0 International license. This is an open access article distributed under the Creative Commons Attribution License which permits unrestricted use, distribution, and reproduction in any medium, provided the original work is properly cited. 


\title{
20 UNDERGRADUATE RESEARCH CRITIQUE
}

\section{Research Critique on "Effects of Finger Taping on Forearm Muscle Activation in Rock Climbers"}

\author{
Sydney Snelbaker \& MinHyuk Kwon \\ California State Polytechnic University, Pomona, CA, USA
}

Keywords: finger taping, flexor tendon pulley, muscle activation

\section{ABSTRACT}

Introduction: A common injury among rock climbers is the flexor tendon pulleys because they use the fingers more than any other sport. Typically, climbers try taping their fingers to aid injury prevention to the flexor tendon pulleys. However, it is unclear the effect of taping on finger muscles activation.

Purpose: The purpose of this critiqued article is to determine if finger taping is effective in reducing flexor tendon pulley injuries in rock climbers by examining the change of muscle activation in the flexor digitorum superficialis (FDS) and flexor digitorum profundus (FDP) muscles.

Methods: Ten expert rock climbers (men, $21.0 \pm 1.7$ years) performed a hanging position for six seconds, requiring a crimp grip and elbow angles between $85^{\circ}$ to $95^{\circ}$ with no tape, $\mathrm{H}$-taped (Schöffl et al (2007), and circular taped (traditional). The angle of their elbows was determined by a 2D Electrical Goniometer (Noraxon). Electromyogram (EMG, Noraxon DTS) was placed on flexor digitorum superficialis (FDS) and flexor digitorum profundus (FDP) muscles of just the subject's right arm to measure muscle activities during trials. The amplitude of FDS and FDP muscles was quantified as root mean square (RMS). Two-way repeated ANOVA Data was performed to determine the differences.

Results: Taping had no significant effect on the muscle activation of the forearm muscles $(p=0.069)$. Additionally, FDS and FDP demonstrated similarity ( $p=0.822)$.

Discussion: Finger taping does not affect the relative muscle activation of the FDS and the FDP muscles during a static hang test of healthy men rock climbers. Therefore, taping may not be an effective measure to prevent flexor tendon pulleys.

Critique: The purpose of this study was to determine if finger taping was an effective preventative measure against finger flexor tendon pulley injuries in rock climbers by comparing involved muscle

Journal of Health, Sports, \& Kinesiology | ISSN 2692-9864 (Online) \& ISSN 2767-5874 (Print) | www.johsk.com 
activity (FDS and FDP). The findings suggested that taping is ineffective in preventing flexor tendon pulley injuries because there was no significant effect in the muscle activation between taped and untapped fingers. The limitations of this study are 1) there were only 10 participants and 2) a hang test is not an adequate representation of rock climbing because rock climbing is a very dynamic activity and sport that utilizes large amounts of muscle control and tendon strain that cannot be depicted from a static hang. In the future, this study should be replicated using more subjects. Muscle activation should be measured while the subjects are vertically climbing. The article states that subject experience may influence the outcome of this study and that they may have different results if their subjects climbed more often compared to an average of 5 hours per week because they are more at risk to injury; I do believe that because they're climbing routes are more challenging that more experienced climbers are at higher risk to injury, however, if the data is to be taken in a controlled setting (lab) then the risk is removed since it is unethical to make a subject participant in anything dangerous. Finally, even though this was the first study to determine if taping could prevent injuries by examining forearm muscle activation, the outcome of this study was expected. All prior studies showed that finger taping is not an effective measure to prevent flexor tendon pulley injury, but instead is more effective in already injured fingers. Overall, I think there is changes that need to be done in order to improve the accuracy of this study; increase participant count and the use of dynamic muscle activation.

\section{Author Information}

Sydney Snelbaker, https://orcid.org/0000-0003-1686-7613

Department of Kinesiology and Health Promotion California State Polytechnic University, Pomona, CA, USA. Email: sydneysnel@aol.com

MinHyuk Kwon, Ph.D., https://orcid.org/0000-0002-7008-7995

Assistant Professor, Department of Kinesiology and Health Promotion California State Polytechnic University, Pomona, CA, USA. Email: minhyukkwon@cpp.edu 
| 2021 | Volume 2 | Issue 3(S) | The Journal of Health, Sports, and Kinesiology |

Journal of Health, Sports, and Kinesiology, 2021, 2(3), 52-53. https://doi.org/10.47544/johsk.202 1.2.3

(c) 2020-2021 by the International Organization for Health, Sports, \& Kinesiology

\title{
21 UNDERGRADUATE RESEARCH CRITIQUE
}

\section{Research Critique on "A Comparison of Velocity-Based and Traditional Percentage-Based Loading Methods on Maximal Strength and Power Adaptations"}

\author{
Casey J. Kimbro, Luis Medina, \& Young Sub Kwon \\ Humboldt Sate University, Arcata, CA, USA
}

Keywords: velocity-based training, percentage-based training, one repetition maximum testing

\begin{abstract}
Introduction: This study explored the differences between percentage and velocity-based training (PBT and VBT) and neuromuscular connection. The exercises used in this test were the back squat, bench press, overhead press, deadlift, vertical jump, and countermovement exercises; these exercises influence neuromuscular stimuli adaptation to resistance training. This study is vital to explore how VBT might be better than PBT for power production and strength improvement.
\end{abstract}

Purpose/objective: This study aimed to determine if VBT yielded better strength adaptations in a 6week resistance training program compared with a traditional PBT program.

Methods: 30 resistance trained men volunteered to participate in this study, assuming that they had at least two prior years of training experience and had been consistently training the last six months. Sixteen men were recruited to participate who met the inclusion criteria. Pre-testing before the study included a countermovement jump (CMJ) test and 1 repetition maximum (RM) testing of the back squat, bench press, overhead press, and deadlift. All 16 men $(22.8 \pm 4.5$ years of age) underwent 6 weeks of resistance training at two sessions per week. Half of the subjects were assigned to VBT, and the other half were assigned to a traditional PBT program. After completing the training program, participants underwent the same testing protocol as before the 6-week program.

Results: No differences between groups were found at the beginning of testing before the 6-week program $(p>0.05)$. However, after 6 weeks of resistance training, the VBT group had a better improvement $(5 \%)$ in the CMJ test than the PBT group (1\%) $(p<0.05)$. In the IRM tests, there were significant increases in strength for the overhead press (VBT and PBT both 6\%), back squat (VBT 9\%, PBT $8 \%)$, deadlift (VBT 6\%), and bench press (VBT 8\%, PBT 4\%) $(p<0.05)$. No significant time relationship was

Journal of Health, Sports, \& Kinesiology | ISSN 2692-9864 (Online) \& ISSN 2767-5874 (Print) | www.johsk.com 
found between groups for the back squat, deadlift, and overhead press ( $p>0.05)$. A significant time relationship was found between groups for the bench press $(p<0.05)$. Furthermore, the VBT group outlifted the PBT group significantly on the back squat, overhead press, and bench press $(p<0.05)$.

Conclusion: The result data presented within this study suggests that using velocity as a training variable may provide greater maximal strength adaptations than traditional PBT loading. Furthermore, a lower total training volume is necessary to improve maximal strength considerably, and more pertinent to the quality of training, allow a favorable adaptation effect to movements including vertical jump.

Critique: This study found that VBT yielded better and faster performance improvements in the CMJ test and all 1RM strength tests. The overall design and methods of the analysis were well organized, but there were a few changes that could have been made. There were initially 30 male subjects who participated in the study, but 16 subjects did not qualify, so 16 more were recruited. The study didn't mention how the authors recruited these subjects, so there is potential for bias in this selection. Furthermore, there was no indication of the athletes' sports background before the study, so it is possible that some athletes had a natural advantage over others due to prior training. Another problem in this study was the exercises selected for the six-week training program. While the overhead press, back squat, deadlift, and overhead press are all straightforward movements to learn, this study compared VBT to PBT. It would have been ideal to include power movements in the athletes' training, such as power cleans, jerks, snatches, and/or broad jumps and plyometrics. It would have also been more balanced to include seated rows or lat pulldowns to work the back. This study included a lot of pressing motions and lacked in pulling movements. Another weak aspect of this study was the lack of inclusion of women. A follow-up study with female subjects would be an excellent comparison to the male subjects' results.

\section{Author Information}

Casey J. Kimbro, https://orcid.org/0000-0001-7892-1449

Department of Kinesiology and Recreation Administration, Humboldt State University, Arcata, California, USA. Email: cjk48@humboldt.edu

Luis Medina, https://orcid.org/0000-0003-2710-3862

Department of Kinesiology and Recreation Administration, Humboldt State University, Arcata, California, USA. Email: Im325@humboldt.edu

Young Sub Kwon, Ph.D., https://orcid.org/0000-0001-8920-2757

Associate Professor, Department of Kinesiology and Recreation Administration, Humboldt State University, Arcata, California, USA. Email: ysk15@humboldt.edu

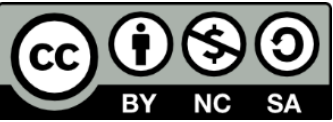

(c) 2021. This work is licensed under a CC BY-NC-SA 4.0 International license.

This is an open access article distributed under the Creative Commons Attribution License which permits unrestricted use, distribution, and reproduction in any medium, provided the original work is properly cited.

Journal of Health, Sports, \& Kinesiology | ISSN 2692-9864 (Online) \& ISSN 2767-5874 (Print)| www.johsk.com 
| 2021 | Volume 2 | Issue 3(S) | The Journal of Health, Sports, and Kinesiology |

Journal of Health, Sports, and Kinesiology, 2021, 2(3), 54-55. https://doi.org/10.47544/johsk.202 1.2.3

(c) 2020-2021 by the International Organization for Health, Sports, \& Kinesiology

\title{
22 UNDERGRADUATE RESEARCH CRITIQUE
}

\section{Research Critique on "Assessment of MTNR1B Type 2 Diabetes Genetic Risk Modification by Shift Work and Morningness-Eveningness Preference in the UK Biobank"}

\author{
Nathan Redmond \& Joon Young Kim \\ Syracuse University, New York, USA
}

Keywords: shift work, MTNR 1B risk allele, type 2 diabetes

\begin{abstract}
Introduction: Rotating night shifts has been correlated with increased T2D risk via circadian misalignment between endogenous circadian cycle and behavioral cycles, potentially affecting worse glucose regulation. The MTNR 1B single nucleotide polymorphism has been associated with T2D as it relates to melatonin rhythms and food intake. Despite the possible linkage between genetic mutation on MTNR IB and consequences of night shift work, there is lack of scientific evidence on their relationships so that it is unknown whether MTNR 1B risk allele could potentially exacerbate T2D risk via the mechanisms linking circadian misalignment.
\end{abstract}

Purpose: Dashti et al. aimed to test (a) the independent association of MTNR 1B risk allele, night shift work, and chronotype on the prevalence of $\mathrm{T} 2 \mathrm{D}$ and $\mathrm{HbA}$ Ic levels, and (b) its interactive effects of MTNR IB risk allele in a large population from the UK Biobank, investigating the respective contributions of genetic predisposition and environmental exposure to the development of chronic disease.

Methods: The present study included 189,488 participants from the UK Biobank as being employed or self-employed and of European decent. The participants self-reported their work schedule and morningness-eveningness preference for chronotype and a genotype containing MTNR 1B risk allele and $\mathrm{HbA}_{1 c}$ levels was obtained. The association between work and morningness-eveningness preference when compared to the prevalence of $\mathrm{T} 2 \mathrm{D}$ and $\mathrm{HbA} \mathrm{A}_{1 \mathrm{c}}$ were evaluated utilizing crude and adjusted linear regression models.

Results: In comparison to day workers, shift working participants had a higher prevalence of T2D (odds ratio [OR] 1.26 [95\% $\mathrm{Cl}$ 1.15-1.39]). The participants who self-reported definite eveningness and those who were classified as definite eveningness from the accelerometer had a significant increase in the prevalence of T2D (OR 1.29 [95\% Cl 1.13-1.47]). Together with increased OR of T2D, higher HbA $\mathrm{H}_{1 \mathrm{c}}$ was observed with shift worker and morningness-eveningness preference. Participants self-reporting

Journal of Health, Sports, \& Kinesiology | ISSN 2692-9864 (Online) \& ISSN 2767-5874 (Print) | www.johsk.com 
definite morningness preference saw an increase in prevalence of the MTNR 1B risk allele (OR 1.17 [95\% $\mathrm{Cl}$ 1.07-1.28]). With each additional G allele (risk allele) in MTNR1B gene, a $10 \%$ increase in T2D risk was observed. However, there was no interaction between shift work and the MTNR IB risk allele.

Conclusion: Comprehensive examination of T2D risk stemmed from genetics and/or environment is essential for better understanding of etiology and optimal identification of early intervention and treatment. In this study, current and night shift work and definite morningness-eveningness has been shown to elevate the prevalence of $\mathrm{T} 2 \mathrm{D}$ and $\mathrm{HbA}$ ic levels, although there was no association between shift work and MTNRIB risk allele. The data suggest that night shift work and misalignment of endogenous circadian rhythm with behavior cycles may put an individual at increased risk of T2D; however, there should be small effects of MTNRIB genetic variation interacting with environmental factor (i.e., night shift) on T2D risk.

Critique: With a growing number of individuals needed to work night shifts, it's especially important to understand the potential increased risk for T2D together with genetic predisposition. However, some limitations in this study hinder the definitive conclusions on the relationship between MTNR IB variants, night shift and T2D risk. First, there was a lack of available data on physical activity, education level and socioeconomic components that should be accounted when examining potential interaction between MTNR IB and environment on T2D risk. Additionally, common night shift jobs include security guard, taxi driver, and emergency room doctors. The health literacy of a security guard may be different than that of an emergency room doctor, thereby skewing the associations with T2D. Patients with low literacy skills may not be able to make good judgements in everyday life concerning eating habits and exercise leading to an increased risk of T2D. Lastly, their cross-sectional design does not allow the investigation of causal relationship. Although the odds of T2D was a primary outcome, not having prospective observation could hinder proper interpretation. In this case, the study may use continuous/sensitive variables of fasting and 2-hr glucose concentrations which are the classical risk biomarkers as their outcome. It is also noted that fasting and 2-hr glucose will provide more accurate risk information than $\mathrm{HbAlc}$ (which the current study used). In closing, MTNR IB has a potential to be investigated as an interactive factor with environment on T2D risk so that further comprehensive examination of T2D pathophysiological components along with its genetic mutation is warranted.

\section{Author Information}

Nathan Redmond, https://orcid.org/0000-0003-0563-3723

Department of Exercise Science, David B. Falk College of Sport and Human Dynamics, Syracuse University, Syracuse, NY, USA. Email: nrredmon@syr.edu

Joon Young Kim, Ph.D., https://orcid.org/0000-0003-0448-1684

Assistant Professor, Department of Exercise Science, David B. Falk College of Sport and Human Dynamics, Syracuse University, Syracuse, NY, USA. Email: ikim291@syr.edu

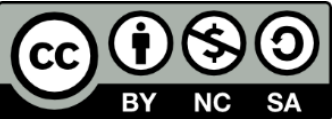

(C) 2021. This work is licensed under a CC BY-NC-SA 4.0 International license.

This is an open access article distributed under the Creative Commons Attribution License which permits unrestricted use, distribution, and reproduction in any medium, provided the original work is properly cited.

Journal of Health, Sports, \& Kinesiology | ISSN 2692-9864 (Online) \& ISSN 2767-5874 (Print)| www.johsk.com 


\title{
23 UNDERGRADUATE RESEARCH CRITIQUE
}

\section{Research Critique on "Effects of Time-Restricted Feeding in Weight Loss, Metabolic Syndrome and Cardiovascular Risk in Obese Women"}

\author{
Sydney Davis \& Joon Young Kim \\ Syracuse University, New York, USA
}

Keywords: obesity, time-restricted feeding, metabolic syndrome, weight loss

\begin{abstract}
Introduction: Obesity has become a serious health concern due to its related comorbidities including metabolic syndrome (MetS), type 2 diabetes, and cardiovascular diseases (CVD). MetS is defined as having at least 3 of the 5 cardiometabolic risk factors including hypertension (systolic blood pressure [SBP] and/or diastolic blood pressure [DBP]), high triglycerides, low HDL-cholesterol, visceral obesity (waist circumference [WC]), and insulin resistance (IR). Time-restricted feeding (TRF) is the process of periods no caloric intake followed by periods of eating with no caloric restrictions. TRF has been studied as a potential weight-loss intervention; however, it is not clear how TRF could deliver beneficial effects on risk reduction of MetS and CVD.
\end{abstract}

Purpose: Schroder et al. aimed to determine the effect of TRF on body composition and the relationship between weight loss and metabolic and cardiovascular risk in middle-aged obese women.

Methods: Obese women (age 35-45 years, $n=32$ ) participated in a non-randomized control trail (NRCT) of TRF for 3 months. The TRF group ( $n=20$, body mass index [BMI] $32.5 \pm 1.1 \mathrm{~kg} / \mathrm{m}^{2}$ ) adopted a regimen of 16 hours without caloric intake and 8 hours of usual caloric intake. The control group $(n=12$, BMI $34.6 \pm 1.2 \mathrm{~kg} / \mathrm{m}^{2}$ ) continued their normal eating habits for 3 months. Anthropometric measurements (BMI and WC), body composition (prediction equations validated by the NHNES), blood pressure, various blood biomarkers including fasting glucose and insulin, 30-year cardiovascular risk evaluation using the Framingham Heart Study in 30 years (CVDRisk30y), and quality of life (WHOQOL questionnaire) were evaluated before and after the intervention.

Results: Both groups were similar in age, BMI, fat mass, WC, IR, and SBP \& DBP, while CVDRisk30y was greater in TRF vs. control (32.1 \pm 7.3 vs. $15.6 \pm 1.8 \%, p<0.001)$. After the 3 months of intervention, there was a $\sim 12 \%$ reduction in CVD risk as measured by the CVDRisk30y in the TRF group (pre 15.6 \pm 1.8 vs. post

Journal of Health, Sports, \& Kinesiology | ISSN 2692-9864 (Online) \& ISSN 2767-5874 (Print) | www.johsk.com 
$13.8 \pm 1.8 \%)$, while the control group remained the same. The reduction of the risk score is accompanied with enhancement of body composition including reduction in body weight, BMl, percent body fat and WC. There was also an increase in quality of life in the TRF group after the 3 months. However even in the TRF group, there was no significant change in the blood biomarkers that are associated with MetS.

Conclusion: The study found that TRF was effective in weight loss, however, there was no significant change in the blood biomarkers that are associated with MetS.

Critique: Despite that the study suggested promising data on TRF as a critical treatment strategy for MetS and CVD, several limitations should be highlighted. Frist, this study was a NRCT thus did not control for potential recruitment/selection biases. Participants could already be a part of a weight loss program, have gained or lost weight recently, or have a predetermined outlook on diet and weight loss. The study did not consider how such biases would affect the results. The control group was informed that they were involved in a diet habit research which may have influenced their 'normal' pattern of eating. Participants also did not track their caloric intake as people's patterns of eating may be drastically different when it comes to amount consumed and meal frequency. This makes it unclear whether the weight loss achieved from the TRF group was from the time restriction itself or from a deficit in caloric intake. Second, as the primary outcome of the study was weight reduction, weight specific QOL (such as OWLQOL) can be used to project TRF effects, rather than using general QOL questionnaire. Lastly, proper use of biomarkers is required in the present study. For instance, HOMA-IR which was analyzed in the present study as an indicator of IR may not be sensitive enough (compared with Triglyceride to HDL ratio) to observe clinical/meaningful difference before and after the 3 months of intervention. Triglyceride to HDL ratio has been accepted as an indirect indicator of IR and it may be responsible to diet intervention. Given that the present study provided potential/preliminary evidence of TRF effects on MetS and CVD, further study with more comprehensive exanimation of the risk factors (i.e., hormone profiles indicating inflammatory response and glycemic control, and preclinical markers of CVD such as pulse wave velocity and intima-media thickness) is warranted.

\section{Author Information}

Sydney Davis, https://orcid.org/0000-0003-3600-9114

Department of Exercise Science, David B. Falk College of Sport and Human Dynamics, Syracuse University, Syracuse, NY, USA.

Email: sydavis@syr.edu

Joon Young Kim, Ph.D., https://orcid.org/0000-0003-0448-1684

Assistant Professor, Department of Exercise Science, David B. Falk College of Sport and Human Dynamics, Syracuse University, Syracuse, NY, USA.

Email: jkim291@syr.edu

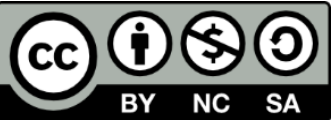

(c) 2021. This work is licensed under a CC BY-NC-SA 4.0 International license. This is an open access article distributed under the Creative Commons Attribution License which permits unrestricted use, distribution, and reproduction in any medium, provided the original work is properly cited.

Journal of Health, Sports, \& Kinesiology | ISSN 2692-9864 (Online) \& ISSN 2767-5874 (Print)| www.johsk.com 
| 2021 | Volume 2 | Issue 3(S) | The Journal of Health, Sports, and Kinesiology |

Journal of Health, Sports, and Kinesiology, 2021, 2(3), 58-59. https://doi.org/10.47544/johsk.2021.2.3

(c) 2020-2021 by the International Organization for Health, Sports, \& Kinesiology

\title{
24 UNDERGRADUATE RESEARCH CRITIQUE
}

\section{Research Critique on "Efficacy and Feasibility of HIIT Training for University Students: The Uni-HIIT RCT"}

\author{
Sewina Yu \& Joon Young Kim \\ Syracuse University, New York, USA
}

Keywords: high-intensity interval training, cardiorespiratory fitness, muscular fitness, cognition, adults

\begin{abstract}
Introduction: High-intensity interval training (HIIT) has gained lots of research interest due to its effectiveness in physiological adaptations for a shorter amount of time relative to traditional aerobic/resistance training. Previous studies investigated the effects of HIIT in a lab setting, thereby lacking evidence on the efficacy of HIIT in a real-world background such as college life. Moreover, focusing on younger adults is unique target population as they have a more positive attitude toward physical activity, thus they have strong intentions to exercise, yet the transition to college life, coursework, and other aspects of this life stage inhibit them from exercising.
\end{abstract}

Purpose: N. Eather et al. aimed to evaluate the efficacy and feasibility of the 8-week HIIT program called "Uni-HIIT" incorporating resistance training in young adult students in a university setting.

Methods: A randomized controlled trial with young adults aged 18-25 (20.4 \pm 1.9 yrs) was performed for the comparison of HIIT training $(n=26)$ vs. control $(n=27)$ over 8 -week periods. The Uni-HIIT consisted of 3 sessions per week lasting 8-12 minutes: each session incorporated both aerobic and corestrengthening exercises, where participants could choose various types of HIIT such as Gym-, Combat-, Brain- and Sports-HIIT. For each HIIT protocol, a 30 second work to 30 second rest ratio was used. Outcomes included cardiorespiratory fitness (20-meter shuttle run), muscular fitness (standing long jump and the push-ups), body composition (skinfold calipers and bioelectrical impedance), executive function (Trail Making Test), anxiety levels (State Trait Anxiety Inventory), and perceived stress (Perceived Stress Scale).

Results: There was a significant group-by-time interaction on cardiorespiratory fitness evidenced by greater improvement on 20-meter shuttle run in the HIIT vs. control (adjusted difference in change [ADC] 8.4 laps, $p=0.004$ ). Further, participants in the HIIT group showed significant increase in (a) upper body muscular fitness (ADC 4.0 reps, $p=0.006$ ) and executive function for Trail B (ADC -5.9, $p=0.052$ ), while both groups exhibited no changes in body composition, lower body muscular fitness, anxiety

Journal of Health, Sports, \& Kinesiology | ISSN 2692-9864 (Online) \& ISSN 2767-5874 (Print)| www.johsk.com 
levels, and perceived stress. Participants rated the program highly in satisfaction (4.73), enjoyment (4.54), and perceived value (4.54).

Conclusion: The Uni-HIIT is considered a novel approach as various "enjoyable" programs are mixed with promising improvements on cardiovascular \& muscular fitness and executive function in young adult students. High satisfaction on the program may attest its feasibility and practicality.

Critique: Despite the uniqueness of various combination of HIIT programs in the university setting, there are several critiques we should discuss for the purpose of developing better HIIT program in younger population. First, metabolic health outcomes such as glucose/insulin metabolism and inflammatory response should be examined in addition to the physical improvement as HIIT aims to improve both cardiometabolic health and sports performance. Second, various protocols for HIIT may be at risk for critical errors (i.e., unmatched amount of the workout between each protocol), which will hinder definitive conclusion of HIIT effects on outcomes. Additionally, the comparison of classical HIIT program is warranted to see if the Uni-HIIT can be a novel approach. Third, low adherence rate for weekly sessions (only $55 \%$ of participants attended at least 2 sessions per week for the 2-weeks) is in fact contradictory to the observation of high ratings in satisfaction (mean $=4.73 / 5$ ), enjoyment (mean $=$ $4.54 / 5$ ), and perceived value (mean $=4.54 / 5$ ). Whether or not the study properly concluded its feasibility is questionable. Lastly, a separation of overweight/obese participants from the lean participants (dominant population in the present study) could minimize potential bias when examining improvements on metabolic health, physical fitness, and athletic performance. In closing, additional investigation of the Uni-HIIT by wide range of weight status and outcome measures and with longer periods of the intervention is warranted.

\section{Author Information}

\section{Sewina Yu}

Department of Exercise Science, David B. Falk College of Sport and Human Dynamics, Syracuse University, Syracuse, NY, USA.

Email: syu125@syr.edu

Joon Young Kim, Ph.D., https://orcid.org/0000-0003-0448-1684

Assistant Professor, Department of Exercise Science, David B. Falk College of Sport and Human Dynamics, Syracuse University, Syracuse, NY, USA.

Email: kim291@syr.edu

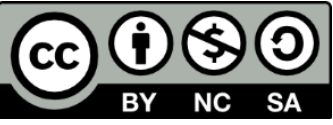

(C) 2021. This work is licensed under a CC BY-NC-SA 4.0 International license.

This is an open access article distributed under the Creative Commons Attribution License which permits unrestricted use, distribution, and reproduction in any medium, provided the original work is properly cited. 
| 2021 | Volume 2 | Issue 3(S) | The Journal of Health, Sports, and Kinesiology |

Journal of Health, Sports, and Kinesiology, 2021, 2(3), 60-61. https://doi.org/10.47544/johsk.2021.2.3

(c) 2020-2021 by the International Organization for Health, Sports, \& Kinesiology

\title{
25 UNDERGRADUATE RESEARCH CRITIQUE
}

\section{Research Critique on "How Diet and Nutrition-Related Mobile Apps Influence Behavior"}

\author{
Vanessa Gonzalez, Eric Serrano, Francis Oppong, \\ Richard Layos, \& Hosung So \\ California State University, San Bernardino, CA, USA
}

Keywords: behavior, mobile apps, behavior mechanisms, diet, nutrition status

\begin{abstract}
Introduction: Obesity has become a severe public health concern in the United States in recent years. Although there are a variety of tactics being used to prevent obesity, the usage of mobile applications is a relatively new discovery that could be effective in assisting people in developing good eating habits. Studies have demonstrated that utilizing health-related applications can successfully lead to weight reduction or weight management behavior change. A number of apps have been developed to assist people in tracking their food intake using calorie counting or food diary methods. The goal of this study is to find out which behavior change factors are linked to the usage of diet and nutritionrelated health apps, as well as if using these apps leads to changes in health behavior.

Objective/ Purpose: The purpose of this study was to find out which behavior change processes are related to the use of diet and nutrition-related health applications, and whether the use of these applications will lead to changes in health behaviors.

Methods: For the study, participants answered questions based on their demographic, their use of nutrition apps, and their diet behavior. For this study, they used a sample of 217 individuals out of 239 participants that met all requirements and completed all their questions. The survey they used made sure that the individuals were at least 18 years of age, lived in the United States, and were able to read English. Questions were based on the three health behavior theories, The health belief model (HBM), theory of planned behavior (TPB), and social cognitive theory (SCT) are three major health behavior change theories for nutrition education.

Results: Overall majority of study participants agreed or strongly agreed with statements about app use enhancing motivation to eat a healthy diet, improving self-efficacy, and increasing willingness to create and achieve healthy diet goals. Furthermore, the majority of participants strongly agreed that using nutritional apps appears to result in changes in their behavior, including increases in actual goal setting to eat a healthy diet $(58.5 \%, 127 / 217)$, constant increase of eating healthy $(57.6 \%, 125 / 217)$,
\end{abstract}

Journal of Health, Sports, \& Kinesiology | ISSN 2692-9864 (Online) \& ISSN 2767-5874 (Print) | www.johsk.com 
and major increase consistency of eating healthy foods $(54.4 \%, 118 / 217)$.

Conclusion: Diet and nutrition-related mobile apps have been shown to be effective instruments for promoting good health habit change. The findings of this study show that using diet/nutrition apps is linked to changes in diet-related behavior. Apps that improve motivation, desire, self-efficacy, attitudes, knowledge, and goal planning, for example, may be highly helpful and beneficial. Theories and constructs known to aid health behavior change, such as those of SCT, TPB, and HBM, should continue to be integrated into health app design and execution to guarantee that mobile apps are effective health behavior change agents.

Critique: According to previous studies, using diet-related apps can lead to better diet and nutrition health behavior modification. Planning, executing, and evaluating treatments are more effective when elements from recognized health behavior change theories are included. Most of the information that was stated is true most apps do help people live a healthier lifestyle. Keeping the sample size small would have been more beneficial, an idea was to compare and contrast if the older generation enjoys more remote fitness apps than the younger generation. Another beneficial idea was to have women and men decide if they enjoy more app diets and develop good health behaviors. This article was well written and strongly agreed with the statement that using diet/nutrition apps increased their motivation to eat a healthy diet. This article has many different research ideas that they can come up with. Most of the participants in this study stated that the use of the Apps boosted their motivation, desire, and ability to modify their nutritional intake. That is a good conclusion that came out of this article because we are always searching for new ideas to try to live a healthier lifestyle. It is shown that health Apps were seen in studies to successfully lead to favorable weight management modifications.

\section{Author Information}

Vanessa J. Gonzalez, https://orcid.org/0000-0001-7417-0705 Email: nessa.gee23@yahoo.com Department of Kinesiology, California State University, San Bernardino, CA, USA.

Eric Serrano, https://orcid.org/0000-0002-5582-239X Email:005965787@coyote.csusb.edu Department of Kinesiology, California State University, San Bernardino, CA, USA.

Francis Oppong, https://orcid.org/0000-0001-9651-0299 Email: 006226879@coyote.csusb.edu Department of Kinesiology, California State University, San Bernardino, CA, USA.

Richard Layos, https://orcid.org/0000-0002--6246-3399 Email:005944532@coyote.csusb.edu Department of Kinesiology, California State University, San Bernardino, CA, USA.

Hosung So, Ph.D. https://orcid.org/0000-0002-3871-2383 Email: hosungso@csusb.edu Professor, Department of Kinesiology, California State University, San Bernardino, CA, USA.

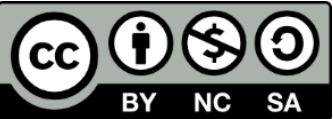

(c) 2021. This work is licensed under a CC BY-NC-SA 4.0 International license.

This is an open access article distributed under the Creative Commons Attribution License which permits unrestricted use, distribution, and reproduction in any medium, provided the original work is properly cited.

Journal of Health, Sports, \& Kinesiology | ISSN 2692-9864 (Online) \& ISSN 2767-5874 (Print)| www.johsk.com 
| 2021 | Volume 2 | Issue 3(S) | The Journal of Health, Sports, and Kinesiology |

Journal of Health, Sports, and Kinesiology, 2021, 2(3), 62-63. https://doi.org/10.47544/johsk.2021.2.3

(c) 2020-2021 by the International Organization for Health, Sports, \& Kinesiology

\title{
26 UNDERGRADUATE RESEARCH CRITIQUE
}

\section{Research Critique on "Effects of Resistance Training on Tendon Mechanical Properties and Rapid Force Production in Prepubertal Children"}

\author{
Humberto Magana, Mason Roach, Matthew Thoe, \& Hosung So \\ California State University, San Bernardino, CA, USA
}

Keywords: resistance training, tendon, children

\begin{abstract}
Introduction: This study investigated the effects of resistance training on human body connective tissue. Within resistance training, the researchers focused the effects resistance training has on tendon mechanical properties and rapid force production in prepubertal children. This study focused on the overall improvements the participating children made to their connective tissues and physical health after participating in the resistance training.

Purpose: The main purpose of this study was to explore the effects of plantar flexor resistant training on the mechanical properties of the Achilles tendon within prepubertal children. In addition, this aimed to examine what mechanisms had a likely chance of adapting to the training.

Methods: Participants included 10 boys and 10 girls who were divided into either control or experimental groups. During the final measurements due to illness and absence each group lost a participant leaving us with a total of 18 active participants. The group went through a resistance training program where groups of no more than 3 completed circuit training with nine stations. Each station a different activity was planned, with plantar flexion resistance training being at one of the nine stations. While the experimental group performed two sets of eight to fifteen the control group had a rest station. Rotations were determined by the completion of one set of repetitions at the plantar flexion RT station by the experimental group or two minutes for the control group's rest period at that station.
\end{abstract}

Results: Achilles' tendon properties, Electromechanical delay, rate force development, and rate of electromyographic were measured at pre- and post-resistance training. Results showed that tendon stiffness and young's modulus increased significantly in the experiment group only ( 29\% and $\sim 25 \%$, respectively). For the experimental group, there was a significant increase in mean training load from the first to last training session by $208 \%$. All other prosperities, however, were not significantly changed.

Journal of Health, Sports, \& Kinesiology | ISSN 2692-9864 (Online) \& ISSN 2767-5874 (Print) | www.johsk.com 
Conclusion: Although this is an indication of changes in the underlying microstructure it may only be hypothesized. Resistance training not only aids in stiffness of tendon allowing for stronger contractions, but it is also an indicator of improved motor performance, coordination, and motor unit activation of the experimental group of participants.

Critique: The synchronous adaptation of tendon to muscle resistance capability must be achieved to efficiently transmit maximal force to the skeletal muscle with minimization of possible injury. These results demonstrate that tendons adapt to heavy resistance training in prepubertal children as they do in adults. The increase in tendon stiffness was accompanied by an increase in the Young's modulus. Increases in the Young's modulus is an indicative of changes in the tendon's underlying microstructure. This may include changes within the tendon's extracellular matrix. This indication also leads to belief that microstructural changes in the tendon most likely underpinned the increase in tendon stiffness and power expressed. The disregard of muscular hypertrophy, Neuromuscular measurements, and sole focus on tendon adaptation bias limits the validity of these findings. Neuromuscular adaptations and muscular hypertrophy must be regarded in the findings that indicate an increase in strength and change in elasticity and motor performance. The equipment utilized is also not adequate due to the angular resistance and being a primary quad based machine (GLPH1 100; Body Solid) and when used for plantar flexion it reduces effectiveness of movement. It should also be considered that a standing calf raise machine (Hammer Strength Hs-SC), or seated calf raise (Hammer Strength PL-CALF) would yield clearer findings. Although this study is a strong indicator of the success of weight training and increased muscular power distribution, it limits credibility due to a need for better program design, variability, and a larger array of measurable results.

\section{Author Information}

Humberto Magana, https://orcid.org/0000-0003-1599-5349

Department of Kinesiology, California State University, San Bernardino, CA, USA.

Email:003131244@coyote.csusb.edu

Mason R. Roach, https://orcid.org/0000-0002-2904-5566

Department of Kinesiology, California State University, San Bernardino, CA, USA.

Email: 006369749@coyote.csusb.edu

Matthew R. Thoe, https://orcid.org/0000-0002-5424-2377

Department of Kinesiology, California State University, San Bernardino, CA, USA.

Email:006034154@coyote.csusb.edu

Hosung So, Ph.D. https://orcid.org/0000-0002-3871-2383

Professor, Department of Kinesiology, California State University, San Bernardino, CA, USA.

Email: hosungso@csusb.edu

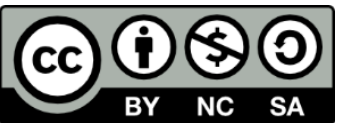

(c) 2021. This work is licensed under a CC BY-NC-SA 4.0 International license.

This is an open access article distributed under the Creative Commons Attribution License which permits unrestricted use, distribution, and reproduction in any medium, provided the original work is properly cited.

Journal of Health, Sports, \& Kinesiology | ISSN 2692-9864 (Online) \& ISSN 2767-5874 (Print)| www.johsk.com 


\title{
27 UNDERGRADUATE RESEARCH CRITIQUE
}

\section{Research Critique on "Stereotypical Views of Beauty and Boys Still Not Letting Girls Play: A Student-Centered Curriculum for Young Girls Through an After-School Activist Approach"}

\author{
Michael Grimes, Cheyenne Wood, Ivan Magana, \\ Moira O'Neill, \& Hosung So \\ California State University, San Bernardino, CA, USA
}

Keywords: body image, elementary, empowerment, Latina, physical education (PE), physical activity (PA)

\begin{abstract}
Introduction: This article explained young women and their physical activity (PA) levels in school due to them feeling uncomfortable, unsafe, or unprotected. As well as these young women feeling very vulnerable at such a young age based on what their parents say and vocalizing their worries about their daughters in physical education (PE) class. It affects these girls when boys tell them they can't participate because they are girls, or they are not good enough. This article goes along with the stereotype that if a girl is pretty, they have nothing else to offer besides their looks, which is very deflating.
\end{abstract}

Purpose: The purpose of this research was to explore how a student-centered curriculum engaged female participants in critical analysis of the "female ideal" and to identify any PA barriers girls face.

Methods: The participants were nine female students that were fifth and sixth graders, and two female coaches who acted as the researchers. Participants met up to twice a week in an after-school program where the student-centered curriculum was held. Data collected consisted of researcher and participant journals, field notes, and semi-structured interviews. Trustworthiness and credibility were established through extended engagement, member checks, peer review, and negative case analysis.

Results: As a result, several girls fail to take part in PA sessions as they experience discomfort and they experience feelings of unsafety, and lack of protection. Moreover, boys prevent girls from playing during PE and PA classes. The researchers see the need to make girls feel more comfortable and

Journal of Health, Sports, \& Kinesiology | ISSN 2692-9864 (Online) \& ISSN 2767-5874 (Print) | www.johsk.com 
encourage them to participate in PA and PE sessions. The researchers also identify other key factors that could play a vital role in influencing girls to participate in PA and PE sessions, including the use of the media. The media could play a significant role in eliminating or reducing the existing stereotypes by representing girls' bodies as more ideal for participating in both PA and PE spaces.

Conclusion: The primary themes that were discussed and analyzed in the study that could significantly affect the participation of girls in PA and programs were boys acting as a barrier to girl's physical participation in afterschool programs and the physical body appearance and structure of girls that could possibly prevent them from participating in certain physical activities. The researchers see the need to make girls feel more comfortable and encourage them to participate in PA and PE sessions.

Critique: The article tried to prove that female participation in PA and PE is problematic due to the involvement of males yet fall short in validity in terms or sample size, age, diversity, location, region, and the focus of their purpose. The sample size was only 9 participants and would be expected to represent all female students in the world. Age is an issue because of the 9 they were all in fifth or sixth Grade 9-12 years old. The age diversity alone would not represent their own school let alone region, state, or country. Diversity was an issue because it was stated most were Latinx and that isn't accurate to the representation of all female students in the school, state, country, or world, with Latinx individuals only accounting for roughly $16 \%$ of the population in the United States of America. The location was an urban school in southern California, United States. That is an issue because the sample doesn't include different geological regions or types of areas other than urban. The article is problematic with its focus due to being too vague whether its purpose was intended to discuss the problem at a city, state, country, or global scale. The article did bring to light a serious potential solvable problem, but just tried to solve it with methods that weren't refined enough or a sample size large enough.

Michael Grimes, https://orcid.org/0000-0002-9579-213X Email: 006863461@coyote.csusb.edu Department of Kinesiology, California State University, San Bernardino, CA, USA.

Cheyenne L. Wood, https://orcid.org/0000-0003-2173-4161 Email: 007357942@coyote.csusb.edu Department of Kinesiology, California State University, San Bernardino, CA, USA.

Ivan Magana, https://orcid.org/0000-0001-6521-0939 Email: 007336141@coyote.csusb.edu Department of Kinesiology, California State University, San Bernardino, CA, USA.

Moira C. O'Neill, https://orcid.org/0000-0001-9554-1456 Email: 005579986@coyote.csusb.edu Department of Kinesiology, California State University, San Bernardino, CA, USA.

Hosung So, Ph.D. https://orcid.org/0000-0002-3871-2383 Email: hosungso@csusb.edu Professor, Department of Kinesiology, California State University, San Bernardino, CA, USA.

()) (1) $\Theta(0)$

(C) 2021. This work is licensed under a CC BY-NC-SA 4.0 International license.

This is an open access article distributed under the Creative Commons Attribution License which permits unrestricted use, distribution, and reproduction in any medium, provided the original work is properly cited. 
| 2021 | Volume 2 | Issue 3(S) | The Journal of Health, Sports, and Kinesiology |

Journal of Health, Sports, and Kinesiology, 2021, 2(3), 66-67. https://doi.org/10.47544/johsk.2021.2.3

(c) 2020-2021 by the International Organization for Health, Sports, \& Kinesiology

\title{
28 UNDERGRADUATE RESEARCH CRITIQUE
}

\section{Research Critique on \\ "Parents and Children's Physical Activity Relationship"}

\author{
Juan Carlos, Kassandra Villalobos, \& Hosung So \\ California State University, San Bernardino, CA, USA
}

Keywords: physical activity, pedometer, role modeling

\begin{abstract}
Introduction: The physical activity (PA) of parents' is often correlated with the PA of their children and is referred to as role modeling, however studies have shown inconsistent results. With the child obesity rate in America at a record high of $19 \%$, finding out whether PA role modeling can increase PA in children or if other variables moderate this relationship is critical.

Purpose: The purpose of this study was to examine the parent-child PA relationship and potential moderators through tracking the amounts of steps taken per day between the parents' and their children.

Methods: A total of 612 7-8-year-olds along with one of their parents volunteered to wear a Steps Count (SC)-T2 pedometer for four consecutive days, consisting of three weekdays and one day on the weekend. Nightly, the participants were required to document their steps within a logbook and reset their pedometer. Self-reported questionnaires were completed by the parent and assessed their PA during the last 7 days along with their child's usual PA. Additional variables such as socioeconomic status (SES), weight status (parent and child), weight status homogeneity, gender (parent and child), and gender homogeneity were evaluated to see whether they impacted the relationship between the PA of the parent and their child. All analyses were done through linear regressions on SPSS.

Results: Overall, once all the data was collected and measured there was significant evidence to suggest a correlation between the PA participation of parents and their children. The results of the pedometer testing showed that for every 1000 steps increased by the parents then the children would take an extra 260 steps. The results were collected as the bivariate, unadjusted Pearson's correlation which showed parents and children's steps to be $r=.25, p<.001$ (partial $r=.24$ ). For the questionnaire portion of the study the PA relationship of parents and children was slightly smaller at (partial $r=.14$ ).
\end{abstract}

Journal of Health, Sports, \& Kinesiology | ISSN 2692-9864 (Online) \& ISSN 2767-5874 (Print)| www.johsk.com 
Boys $(M=9,075, S D=4,832)$ took more steps than girls $(M=8,095, S D=4,507)$, while there was no significant difference between mothers $(M=7,773, S D=3,136)$ and fathers $(M=7,568, S D=7,737)$ in steps per day.

Conclusion: Rising rates of child obesity in America have continuously grown over the past decades and will continue to grow unless changes are made. The data clearly shows significant correlations between the amount of PA between parents and children and why it is important to not only have children be active on their own but also set the example as parents and be active alongside them. The data shows the increase of PA in children when they have parents modeling the desired behavior, in this instance with the pedometer step count per day. Setting this type of example for children at a young age can help lead them down a healthier lifestyle filled with less health issues as they continue to grow.

Critique: Although the findings of this study showed a highly significant relationship between the PA of the parents' and their children while evaluating additional potential moderators, this study does not prove a causal relationship. By objectively measuring the PA with pedometers, it reduces biases. Contradictory, using pedometers to measure PA can lead to inaccurate results even though the SCT2 pedometers, used within this study, are shown to be reliable and accurate. For instance, this study relied solely on the logbooks and unknowingly the participants could track inaccurate steps taken throughout the day and the pedometers do not track missing wear time. The use of pedometers can also increase the participants' activity levels since they know they are being monitored and with this study consisting of only four days, there is uncertainty of whether the PA reported is from an initial increase. Lastly, most of the parents within the study had a greater education and were from highincome families. Previous studies have shown that parents with a greater education along with being high-income earners tend to be more physically active due to more resources available. Therefore, a longitudinal objective study would need to be done on a diverse population to develop a causal relationship.

\section{Author Information}

Juan J. Carlos, https://orcid.org/0000-0003-3092-0564

Department of Kinesiology, California State University, San Bernardino, CA, USA.

Email: 006956073@coyote.csusb.edu

Kassandra Villalobos, https://orcid.org/0000-0003-2199-9813

Department of Kinesiology, California State University, San Bernardino, CA, USA.

Email:006288356@coyote.csusb.edu

Hosung So, Ph.D. https://orcid.org/0000-0002-3871-2383

Professor, Department of Kinesiology, California State University, San Bernardino, CA, USA.

Email: hosungso@csusb.edu

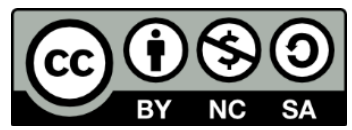

(C) 2021. This work is licensed under a CC BY-NC-SA 4.0 International license.

This is an open access article distributed under the Creative Commons Attribution License which permits unrestricted use, distribution, and reproduction in any medium, provided the original work is properly cited.

Journal of Health, Sports, \& Kinesiology | ISSN 2692-9864 (Online) \& ISSN 2767-5874 (Print)| www.johsk.com 
| 2021 | Volume 2 | Issue 3(S) | The Journal of Health, Sports, and Kinesiology |

Journal of Health, Sports, and Kinesiology, 2021, 2(3), 68-69. https://doi.org/10.47544/johsk.2021.2.3

(c) 2020-2021 by the International Organization for Health, Sports, \& Kinesiology

\title{
29 UNDERGRADUATE RESEARCH CRITIQUE
}

\section{Research Critique on "Health-Related Physical Fitness and Physical Activity in Elementary School Students"}

\author{
Bobby Wiley \& Hosung So \\ California State University, San Bernardino, CA, USA
}

Keywords: physical activity, physical fitness, pedometer

\begin{abstract}
Background: This study examined the associations between physical activity (PA) and physical fitness. This study also focused on if being in better physical shape directly correlate's to being more physically active in different settings in both boys and girls. Currently almost $1 / 3$ of all US children are overweight or obese, these stats from our K-12 schools. Studies show that children are $15 \%$ less physically active than their parents were when they were a kid.

Purpose: The purpose of this study is to examine students in different physical fitness tests to determine their physical fitness levels During various settings with the students having different physical fitness levels and different physical fitness activity throughout their normal daily lives. This will determine if being physically active will determine your physical fitness level.

Methods: A total of 265 fifth grade students with an average age of 11 volunteered to participate in this study. The student's physical fitness was measured by 4 Fitness Grams tests which included Progressive Aerobic Cardiovascular Endurance Run (PACER), curl-up, push-up, and trunk lift tests. The student results were measured over seven days in different settings and the data was analyzed with descriptive statistics to determine results.

Results: Results show that the students PA minutes were significantly associated with the time spent in physical education class or recess. Most of the students did not spend time doing sports or extracurricular activities outside of school, which affected their physical fitness level. 106 boys whose fitness scores were 62 or greater were classified as having healthy fitness levels. While the remaining boys were classified as having unhealthy fitness levels which correlated to lower scores in their fitness tests. For girls 112 who scored 50 or greater were classified as having healthy fitness levels who are the other 20 girls scored lower than 50 and were classified as having unhealthy fitness levels. It is
\end{abstract}

Journal of Health, Sports, \& Kinesiology | ISSN 2692-9864 (Online) \& ISSN 2767-5874 (Print) | www.johsk.com 
documented that girls are less physically active than boys, this can show why the score for being physically fit was reduced to 50 versus 62 for boys.

Conclusion: As expected, the results show that students that armor physically active in school and active at home as well had better fitness levels. The study shows that children's healthy cardiovascular endurance is mostly correlated to their total weekly physical activity. With cardiovascular continuing to be a problem in our country this should be a focal point for our children to be more physically active in school during recess and PE class but also to be active at home with their parents or any other extracurricular activities. With monitoring our children weekly physical activity could lower the risk of cardiovascular problems for them. With all the data captured from the cardiovascular endurance and the muscular strength tests, it shows better results with physical activity throughout the seven-day trial these children were examined.

Critique: This article proves that children need to be more physically active. In this study the students that participated and volunteered for the study were overwhelmingly more physically fit. With only 27 boys and 20 girls scoring low physical fitness levels it leaves majority of the students in the study to be considered physically fit in this study. With the statistics given most children at an average age of 11 are not physically fit so l'd argue that in this volunteered study the participants just so happened to be physically fit, out of the 352 students the stats show that those students we're more than likely under the unhealthy physically fit category. Which would change the data that was collected to be more in favor of the unhealthy fitness level group. Overall, the study did show a correlation that healthy physical activity in your daily life usually means healthy physical fitness levels or vice versa.

\section{Author Information}

Bobby Wiley, https://orcid.org/0000-0002-0676-4082

Department of Kinesiology, California State University, San Bernardino, CA, USA.

Email: 006776270@coyote.csusb.edu

Hosung So, Ph.D. https://orcid.org/0000-0002-3871-2383

Professor, Department of Kinesiology, California State University, San Bernardino, CA, USA.

Email: hosungso@csusb.edu

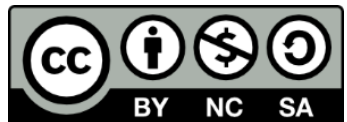

(C) 2021. This work is licensed under a CC BY-NC-SA 4.0 International license.

This is an open access article distributed under the Creative Commons Attribution License which permits unrestricted use, distribution, and reproduction in any medium, provided the original work is properly cited.

Journal of Health, Sports, \& Kinesiology | ISSN 2692-9864 (Online) \& ISSN 2767-5874 (Print) | www.johsk.com 


\title{
30 UNDERGRADUATE RESEARCH CRITIQUE
}

\section{Research Critique on "Perspectives of Students with Disabilities Toward Physical Education: A Qualitative Inquiry Review"}

\author{
Katherine Walker, Christian Briceno, Shaun Cannady, \& Hosung So \\ California State University, San Bernardino, CA, USA
}

Keywords: attitudes, disability, inclusion, special needs population

\begin{abstract}
Introduction: Up until the signing of the Americans with Disabilities Act in 1990, disabled individuals continued to have a hard time properly expressing themselves. The goal of this study was to explore the varying perspectives of students with disabilities toward physical education classes. The focal points of their desired perspectives include; views toward averagely maturing individuals, views toward the physical education teachers, and views toward the inclusivity and exclusivity of the class. This research gives an insight to a broad spectrum of physical educators on how students with differing abilities experience physical education classes.

Purpose: The main purpose of this article was to review and examine previously published qualitative inquiries in order to understand the perspective of students with disabilities toward physical education.

Methods: This qualitative inquiry employs a systematic review process in which a handful of research is already conducted and attempts to incorporate the findings. Five different steps were used including; focusing on a specific question ("i.e., what has the available qualitative inquiry said about the perspectives of children with disabilities about their physical education experiences"; Haegele \& Sutherland, 2015), had a specific protocol that would help with accompanying processes ("i.e. the protocol was developed to include peer-reviewed, qualitative inquiry pertaining to this topic"; Haegele \& Sunderland, 2015), found correlating literature through an extensive search, made specific decisions regarding inclusion and exclusion, and compiled the research findings while continuing to be thorough and straightforward. They specifically used search engines incorporating but are not limited to Academic Search Complete, Education Full Text, Education Research Complete, and more. The searches consisted of words such as: disability, disabled, special needs; physical education; and qualitative research, qualitative method, naturalistic method, perceptions and perspectives. Going through the inclusion and exclusion criterion, researchers listed what they believed should be included in the articles in order to be considered for the qualitative inquiry and what should be excluded as a
\end{abstract}

Journal of Health, Sports, \& Kinesiology | ISSN 2692-9864 (Online) \& ISSN 2767-5874 (Print) | www.johsk.com 
means to narrow down potential findings. The search process found 228 articles, 199 were excluded due to not meeting all inclusion criteria thus leaving 29 qualifying studies. The final thirteen articles that were chosen for the inquiry focused on the perspectives of students with disabilities. The articles were contingent on a narrative analysis. They were read over multiple times in an attempt to discover similarities and differences between the major findings in each article. Haegele and Sunderland then decided on three thematic clusters and themes from the thirteen articles.

Results: Research produced thirteen studies that focused on the perspectives of students with disabilities. The thirteen studies had three major similarities picked by the researchers, Haegele and Sunderland. The two found these similarities to be views toward averagely maturing students, views toward physical education teachers, and views toward the feelings of inclusion and exclusion in physical education courses.

Conclusion: It is important for educators to understand the perspectives of students with disabilities. By trying to acknowledge how these students feel could allow them to have a better experience in their physical education courses which in turn lifetime physical activity.

Critique: The general search of the article allowed for a wide variety of published studies to be considered. It was beneficial of the authors to use three specific themes to focus on when reading through the studies which posed a common theme of all thirteen. The article displayed great details involving the true perspective and feelings of the kids with disabilities. They performed many studies to find out the results which described the pros and cons of each result. By including how kids with disabilities portrayed themselves around other students and the impacts with teachers shows outstanding feedback. The authors should have mentioned the way prior experiences within physical education could have possibly affected their current attitudes, positive or negative.

\section{Author Information}

Katherine E. Walker, https://orcid.org/0000-0002-6288-9402 Email: 007785785@coyote.csusb.edu Department of Kinesiology, California State University, San Bernardino, CA, USA.

Christian Briceno, https://orcid.org/0000-0002-8464-8343 Email:005936901@coyote.csusb.edu Department of Kinesiology, California State University, San Bernardino, CA, USA.

Shaun M. Cannady, https://orcid.org/0000-0001-9540-9690 Email: shauncannadyrock@gmail.com Department of Kinesiology, California State University, San Bernardino, CA, USA.

Hosung So, Ph.D. https://orcid.org/0000-0002-3871-2383 Email: hosungso@csusb.edu Professor, Department of Kinesiology, California State University, San Bernardino, CA, USA.

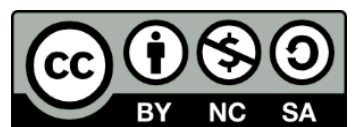

(C) 2021. This work is licensed under a CC BY-NC-SA 4.0 International license.

This is an open access article distributed under the Creative Commons Attribution License which permits unrestricted use, distribution, and reproduction in any medium, provided the original work is properly cited. 


\section{RESEARCH GRANT REPORT}

\section{Effects of Ketogenic Dietary Intervention on Anthropometrics, Body Composition, Metabolic \\ Parameters, and Psychological Factors in Young Obese Population.}

Hayden West \& Soon-Mi Choi

Midwestern State University, Texas, USA

Keywords: ketogenic diet, body composition, metabolic parameters, psychological factors, young obese

\section{Introduction}

Obese have a significantly higher Body Mass Index (BMI), which can be associated with poor nutritional intake and sedentary lifestyles. The ketogenic diet is a form of a dietary intervention that is often implemented for metabolic syndrome individuals such as obese populations. Ketogenic diet is described as high in dietary fat, adequate protein, and constricted numbers of carbohydrates to achieve heightened production of ketone bodies. Reasoning for ketogenic dietary intake for decreased carbohydrates are direct impacts on glycemia levels, which permits increased rate of fat mass loss.

\section{Purpose}

The purpose of this study was to investigate the effects of a ketogenic diet on anthropometrics, body composition, metabolic parameters, and psychological factors in young obese population.

\section{Methods}

Seven obese participants ( $\mathrm{n}=7$, height $174.8 \pm 10.9 \mathrm{~cm}$, weight $105 \pm 20.7 \mathrm{~kg}$, BMl $34.6 \pm 4.8 \mathrm{~kg} \cdot \mathrm{m}^{-2}$ ) completed an 8-week intervention with a 70:20:10 ratio of fats to proteins to carbohydrates. Participants were provided three meals per day for 8 weeks. Three testing sessions were provided during the intervention. The first session allowed for familiarization of testing protocols and providing consent forms to participants. The second and third sessions were used to determine pre- and posttests measurements. Weight $(\mathrm{kg})$ and height $(\mathrm{cm})$ were measured using a stadiometer beam scale (Health O Meter 420KL, Columbia, MD), and BMI was calculated. Flexible tape was used to measure both waist and hip circumferences, which were used to determine hip to waist circumference ratios.

\section{Journal of Health, Sports, \& Kinesiology | ISSN 2692-9864 (Online) \& ISSN 2767-5874 (Print) | www.johsk.com}


Skin fold calipers were used to measure body fat percentage through the Jackson-Pollock 3-site method. Total ketone levels were recorded by urinary ketone test strips in every day for 8 weeks. A metabolic analyzer (Cardio Coach $\mathrm{CO}_{2}$ Model 9002- $\mathrm{CO}_{2}$ ) was used to measure resting metabolic rate (RMR) to determine caloric intake amounts and the respiratory exchange ratio for each participant. Participants were instructed to be in a fasted state for 8 hours before the urinary ketone and resting metabolic rate tests. The modified Bruce protocol treadmill test was used to measure relative maximal oxygen consumption $\left(\mathrm{VO}_{2 \mathrm{max}}\right)$ due to the physical constraints placed by participants being classified as obese. Psychological factors were measured by the Medical Outcomes Study Questionnaire Short Form 36 Health Survey. Statistical analyses were performed with IBM Statistical Package for Social Science (SPSS 27.0, SPSS Inc., Chicago, USA). All data was reported as mean and standard deviation (SD). Dependent paired t-Test was used to determine ketogenic diet intervention effects. Frequencies were used to measure results from psychological factors. Statistical significance was set a priori $p \leq 0.05$.

\section{Results}

Participants within the study noted significant reductions in anthropometric variables during 8 weeks: body mass (Pre: $105.8 \pm 20.5 \mathrm{~kg}$ Post:98.9 $\pm 18.8 \mathrm{~kg}, \mathrm{p}=0.000$ ), BMI (Pre: $34.6 \pm 4.8 \mathrm{~kg} \cdot \mathrm{m}^{-2}$, Post:32.2 \pm 4.2 $\mathrm{kg} \cdot \mathrm{m}^{-2}, \mathrm{p}=0.001$ ), waist circumference (Pre: $101.5 \pm 13.9 \mathrm{~cm}$, Post: $96.3 \pm 13.3 \mathrm{~cm}, \mathrm{p}=0.000$ ), and hip circumference (Pre: $112.6 \pm 11.5 \mathrm{~cm}$, Post: $107.3 \pm 10.8 \mathrm{~cm}, p=0.000$ ). Significant reductions were shown in body composition variables: body fat (Pre: $25.6 \pm 0.8 \%$, Post: $21.1 \pm 1.4 \%, p=0.000$ ), and lean body mass (Post: $78.9 \pm 14.9 \mathrm{~kg}$, Post: $78.2 \pm 14.5 \mathrm{~kg}, \mathrm{p}=.0035$ ). Significant reductions were shown in metabolic parameters: systolic blood pressure (Post: $126.6 \pm 10.0 \mathrm{mmHg}$, Post: $120 \pm 6.6 \mathrm{mmHg}, p=0.029$ ), diastolic blood pressure (Pre: $81.7 \pm 4.9 \mathrm{mmHg}$, Post: $76.3 \pm 1.8 \mathrm{mmHg}, \mathrm{p}=0.020$ ), and $\mathrm{VO}_{2 \max }$ (Pre: $47.6 \pm 8.9$ $\mathrm{mL} \mathrm{kg}^{-1} \cdot \mathrm{min}^{-1}$, Post: $51.8 \pm 9.2 \mathrm{~mL} \mathrm{~kg}^{-1} \cdot \mathrm{min}^{-1}, \mathrm{p}=0.001$. There were no significant changes in ketone bodies $(p=0.090)$, resting metabolic rate $(p=0.150)$, and resting heart rate $(p=0.177)$. Overall, the psychological questionnaire measured quality of life factors such as feelings of energy, satiety, and emotional health. Questionnaire was recorded by weekly, pre- and post-intervention as well. Question 1 within the psychological questionnaire results shown a reduction in the negative aspect in poor health score, with a significant increase shown towards good health. Question $3 \mathrm{~g}$ results shown that here was a significant increase in frequencies of improvement towards having no limitations regarding walking a one-mile distance. Question $9 \mathrm{~g}$ had significant increase in individuals selecting improved overall energy levels in comparison to baseline.

\section{Discussion and Conclusion}

The 8 weeks of ketogenic diet intervention affected positively in anthropometrics, body composition, metabolic and psychological factors. The main finding was the reduction of total fat mass through bioenergetic pathways. During nutritional ketosis, the main source of energy production comes from the use of fat, high in density, as a primary fuel source versus normal reliance of carbohydrate consumption and conversion into glucose. In obese populations, changes in physical activity associated within a diet intervention may have a significant impact on body mass reductions. The primary impact of the ketogenic diet combined with the synergistic effect of increased physical activity can result in fluctuations to anthropometrics, body composition, metabolic parameters, and psychological factors. Total fat mass reductions allow for a reduction in overall weight and circumferences measures. Significant lean body mass reduction can be associated with the

Journal of Health, Sports, \& Kinesiology | ISSN 2692-9864 (Online) \& ISSN 2767-5874 (Print)| www.johsk.com 
adaptation process of consuming a ketogenic diet, along with no exercise training protocols combined with the dietary intervention. In metabolic parameters, significant changes were shown due to decreases in body mass that made to improve exercise capacity. No significant changes were shown in absolute resting metabolic rate, resting heart rate, and ketone bodies, which is typical of research with no training interventions. Relative resting metabolic rate $\left(\mathrm{L} \mathrm{kg}^{-1} \cdot \mathrm{min}^{-1}\right)$ was not shown significant changes due to the retention of total caloric expenditure with the decrease in body mass.

Significant changes noted in physical variables have a direct relationship on the influence of psychological factors. Psychological questionnaire values were represented by Likert scale indicating frequencies, allowing for a comparison of baseline, individual weeks, and post-intervention. Lowered scores were noted following implementing intervention, related to the decrease in overall energy and mental association with physical health. Following initial intervention adaptations, scores steadily increased until reaching initial values. Psychological questionnaires values improved in comparison to baseline values following completion of dietary intervention.

In conclusion, the 8 weeks of ketogenic diet intervention may contribute or change bioenergetics pathways and it results in significant adaptations on anthropometrics, body composition, metabolic parameters, and psychological factors in young obese population.

\section{References}

Al-Khalifa, A., Mathew, T. C., Al-Zaid, N. S., Mathew, E., \& Dashti, H. M. (2009). Therapeutic role of lowcarbohydrate ketogenic diet in diabetes. Nutrition (Burbank, Los Angeles County, Calif.), 25(11-12), 1177-1185.

Arroyo-Johnson, C., \& Mincey, K. D. (2016). Obesity epidemiology worldwide. Gastroenterology Clinics of North America, 45(4), 571-579.

Batch, J. T., Lamsal, S. P., Adkins, M., Sultan, S., \& Ramirez, M. N. (2020). Advantages and disadvantages of the ketogenic Diet: A Review Article. Cureus, $12(8)$, e9639.

Brinkworth, G. D., Noakes, M., Clifton, P. M., \& Buckley, J. D. (2009). Effects of a low carbohydrate weight loss diet on exercise capacity and tolerance in obese subjects. Obesity, 17(10), 19161923.

Bueno, N. B., de Melo, I. S. V., de Oliveira, S. L., \& da Rocha Ataide, T. (2013). Very-low-carbohydrate ketogenic diet $\mathrm{V}$. low-fat diet for long-term weight loss: A meta-analysis of randomized controlled trials. British Journal of Nutrition, 110(07), 1178-1 187.

Cantrell, C. B., \& Mohiuddin, S. S. (2020). Biochemistry, ketone metabolism. PubMed; StatPearls Publishing. https://www.ncbi.nlm.nih.gov/books/NBK554523/

Castro, A. I., Gomez-Arbelaez, D., Crujeiras, A. B., Granero, R., Aguera, Z., Jimenez-Murcia, S., Sajoux, I., Lopez-Jaramillo, P., Fernandez-Aranda, F., \& Casanueva, F. F. (2018). Effect of a very lowcalorie ketogenic diet on food and alcohol cravings, physical and sexual activity, sleep disturbances, and quality of life in obese patients. Nutrients, 10(10), 1348.

Chang, C., Borer, K., Lin, P., (2017). Low-Carbohydrate-High-Fat Diet: Can it help exercise performance? Journal of Human Kinetics, 56(1), 81-92.

Cox, P. J., Kirk, T., Ashmore, T., Willerton, K., Evans, R., Smith, A., Murray, A. J., Stubbs, B., West, J., McLure, S. W., King, M. T., Dodd, M. S., Holloway, C., Neubaver, S., Drawer, S., Veech, R. L., Griffin, J. L., \& Clarke, K. (2016). Nutritional ketosis alters fuel preference and thereby endurance performance in athletes. Cell metabolism, 24(2), 256-268.

Journal of Health, Sports, \& Kinesiology | ISSN 2692-9864 (Online) \& ISSN 2767-5874 (Prinf) | www.johsk.com 
Dashti, H. M., Mathew, T. C., Khadada, M., Al-Mousawi, M., Talib, H., Asfar, S. K., Behbahani, A. I., \& AlZaid, N. S. (2007). Beneficial effects of ketogenic diet in obese diabetic subjects. Molecular and Cellular Biochemistry, 302 (1-2), 249-256.

Dhillon, K. K., \& Sonu Gupta. (2019). Biochemistry, ketogenesis. Nih.gov; StatPearls Publishing. https://www.ncbi.nlm.nih.gov/books/NBK493179/

Dostal, T., Plews, D. J., Hofmann, P., Laursen, P. B., \& Cipryan, L. (2019). Effects of a 12-Week very-low carbohydrate high-fat diet on maximal aerobic capacity, high-intensity intermittent exercise, and cardiac autonomic regulation: Non-randomized parallel-group study. Frontiers in physiology, 10, 912.

Ebbeling, C. B., Feldman, H. A., Klein, G. L., Wong, J., Bielak, L., Steltz, S. K., Luoto, P. K., Wolfe, R. R., Wong, W. W., \& Ludwig, D. S. (2018). Effects of a low carbohydrate diet on energy expenditure during weight loss maintenance: Randomized trial. BMJ (Clinical research ed.), 363, k4583.

Evans, M., Cogan, K. E., \& Egan, B. (2017). Metabolism of ketone bodies during exercise and training: Physiological basis for exogenous supplementation. The Journal of Physiology, 595(9), $2857-$ 2871.

Friedman, M. I., \& Appel, S. (2019). Energy expenditure and body composition changes after an Isocaloric ketogenic diet in overweight and obese men: A secondary analysis of energy expenditure and physical activity. PLOS ONE, 14(12), e0222971.

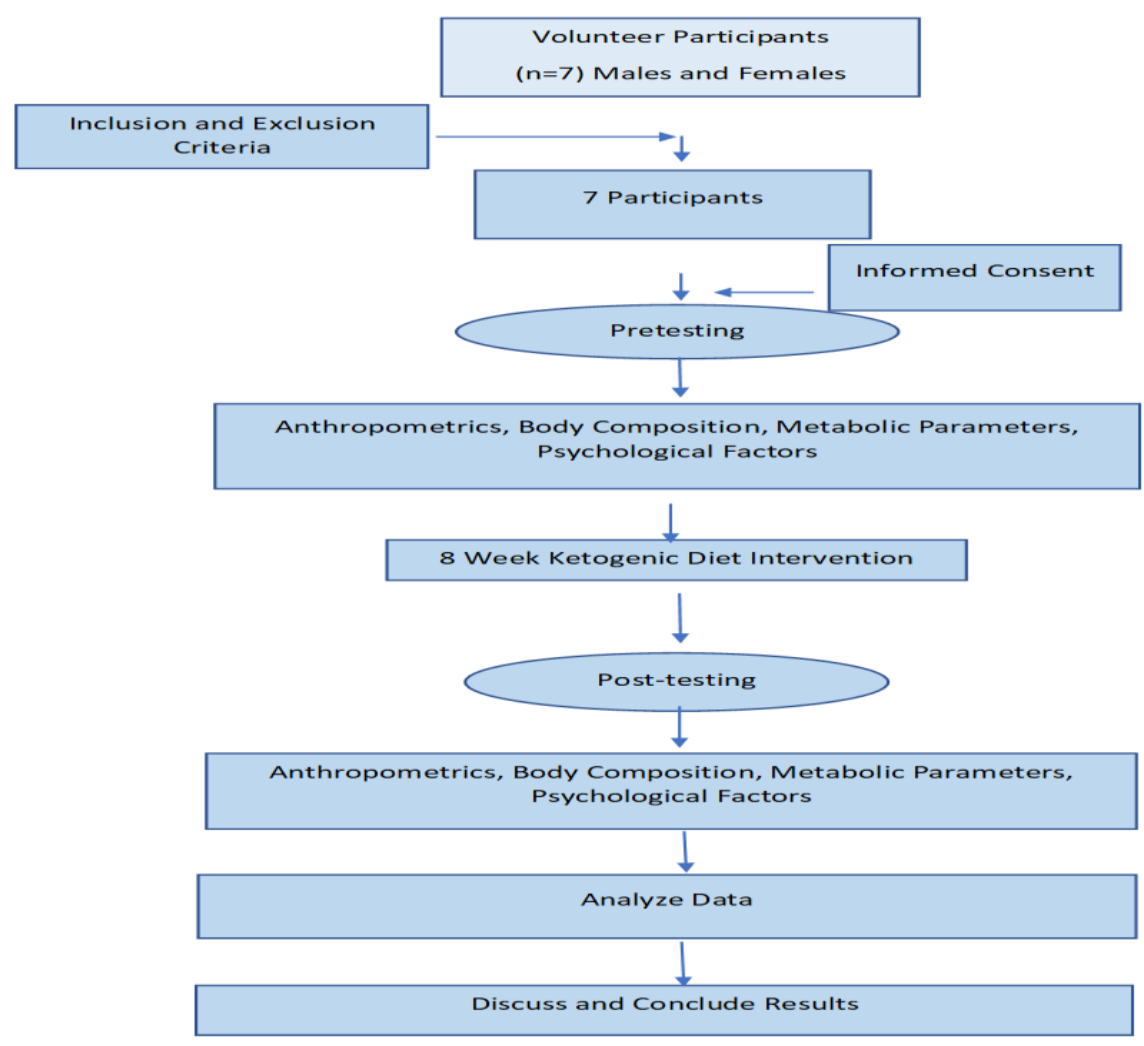

Figure 1. Study Design.

Journal of Health, Sports, \& Kinesiology | ISSN 2692-9864 (Online) \& ISSN 2767-5874 (Print) | www.johsk.com 
| 2021 | Volume 2 | Issue 3(S) | The Journal of Health, Sports, and Kinesiology |

\section{Author Information}

Hayden West, https://orcid.org/0000-0002-5538-5273

Graduate Student, Department of Athletic Training \& Exercise Physiology, Robert D. \& Carol Gunn College of Health Science \& Human Services, Midwestern State University, Wichita Falls, TX, USA.

Email: hayden.west35@yahoo.com

Soon-Mi Choi, Ph.D., https://orcid.org/0000-0002-2796-2061

Associate Professor, Department of Athletic Training \& Exercise Physiology, Robert D. \& Carol Gunn College of Health Science \& Human Services, Midwestern State University, Wichita Falls, TX, USA.

Email: $\underline{\text { soonmi.choi@msutexas.edu }}$

\section{Funding/ IRB Acknowledgement}

Scholarship funding was provided by the IOHSK 2021 Graduate Student Research Grant. Remaining funds were self-funded by the researcher. IRB approval institution is Midwestern State University. IRB approval number is 20120301.

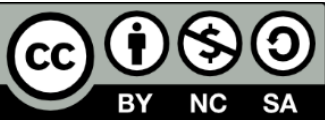

(C) 2021. This work is licensed under a CC BY-NC-SA 4.0 International license.

This is an open access article distributed under the Creative Commons Attribution License which permits unrestricted use, distribution, and reproduction in any medium, provided the original work is properly cited. 
| 2021 | Volume 2 | Issue 3(S) | The Journal of Health, Sports, and Kinesiology |

Journal of Health, Sports, and Kinesiology, 2021, 2(3), 77-81. https://doi.org/10.47544/johsk.2021.2.3

(c) 2020-2021 by the International Organization for Health, Sports, \& Kinesiology

\title{
32 RESEARCH GRANT REPORT
}

\section{Effects of VR Games on Mental Health Among College Students: A Review of Literature}

\author{
Se Ik Park, Eun-Ok Baek, \& Hosung So
}

California State University, San Bernardino, CA, USA

Keywords: virtual reality, instructional technology, physical education, VR game, mental health

\section{Abstract}

This article aims to conduct a literature review on how VR games affect the mental health of college students. The review was systematically conducted to identify articles on this topic. A total of seven papers related to mental health were identified and these studies proved that $V R$ games are effective against depression, anxiety, enjoyment, and stress. The review of literature found that research on VR games is still insufficient and needs to be studied and more research in the future.

\section{Introduction}

For decades, VR-related technologies and quality have improved. With the advancement of this technology, people can have a different experience in the new world. People have the opportunity to experience various experiences in a world other than the real world. Kyaw et al (2019) noted that virtual reality (VR) is a technology that allows users to acquire practical knowledge by exploring and manipulating real or artificial three-dimensional multimedia sensory environments created by computers in real-time.

VR technology is expanding to various fields such as education, games, and medical care, and has a significant impact. Many educational institutions are challengingly adopting VR classes and applying them to education using technology. However, despite the increase in interest and value of $V R$, research on the effectiveness of VR games is still insufficient.

\section{Purpose}

The goal of this study is to review various ranges of literature on the effectiveness of VR games on mental health and to know how VR games will be effective for college students in mental health. 
| 2021 | Volume 2 | Issue 3(S) | The Journal of Health, Sports, and Kinesiology |

\section{Methodology}

This paper conducts a literature review. Wee and Banister (2015) have noted that literature reviews are highly useful for both researchers and readers, as they can provide well-organized, up-to-date overviews and add value to the studies. According to Denny and Tewksbury (2012), literature reviews are important because authors can use them to access a wealth of information about their subjects of interest and gain knowledge of prior research to strengthen their writing. In addition, literature reviews are key resources for ensuring the reliability and integrity of an author's argument.

Inclusion criteria: Criteria were specified to select appropriate studies to include in the review. First, the data needed to relate to VR games. Second, studies had to include both VR games and mental health term (e.g., disability, anxiety, stress) as keywords.

Study selection: Approximately 160 potentially relevant studies were found. Titles, keywords, and abstracts were reviewed to identify articles that met the qualification criteria. The selection excluded duplicate papers, studies on VR subjects beyond the scope of the study (e.g., VR game development), and literature that was not related to mental health. Ultimately, seven studies fulfilled the eligibility criteria.

\section{Results}

The literature review yielded numerous findings concerning the effects of VR games on mental health. For instance, Shaw and Lubetzky (2021) have investigated whether short VR exercise games can reduce stress and anxiety in adolescents. In their experiment, two groups (VR group $n=16$, non-VR group $n=14$ ) were randomly assigned to play similar dodgeball games for 10 minutes. The authors assumed that VR exercise would offer benefits, but both groups had less anxiety and lower stress scores after 10 minutes of exercise (modulate Effect Size).

In order to prevent negative mental health in the future, Rodriguez et al (2015) introduced a Gameteen (GT) system and conducted a study by selecting adolescents $(n=51)$ between the ages of 9-14 to find out the effects of emotional regulation strategies such as depression and anxiety through VR-based serious games. As a result, it was found that VR-based GT systems are sufficiently effective tools for ER strategies related to mental health.

With the aim of reducing anxiety and destructive behavior, Bossenbroek et al. (2020) have conducted a single case study experiment with secondary school students $(n=8)$ for VR games. The participants completed a VR biofeedback game session over a four-week period. Data were collected through interviews and post-questionnaires. The authors detected significant decreases in anxiety and destructive classroom behavior among participants.

In research by Song et al. (2015), stroke patients $(n=40)$ were randomly divided into virtual reality group and ergometer training group to compare their effects on psychological and physical characteristics. During the eight-week experiment, the researchers observed significant improvement $(p<0.05)$ in psychological characteristic scores (Beck Depression Inventory and the relationship change scale) in both groups. In particular, the VR game groups demonstrated more significant improvement

Journal of Health, Sports, \& Kinesiology | ISSN 2692-9864 (Online) \& ISSN 2767-5874 (Print)| www.johsk.com 
compared to the other groups. Lee et al. (2012), who are cited in this paper, have also noted that engagement with VR games can mitigate depression. Shin et al. (2015) have conducted a similar randomized controlled group study in which stroke patients were assigned to one of two groups (VR+OT, OT). The researchers evaluated participants by using Short Form Health Survey-36 (SF-36) to collect data on health-related quality of life (HRQOL). Studies have indicated that game-based VR rehabilitation and existing occupational therapy (OT) can improve specific HRQOL items, depression, and upper limb functions in chronic stroke patients.

Yen et al. (2021) have performed a study to improve mental health through VR exercise games in participants aged 60 or older. Based on 18 randomized tests, the findings suggest that VR exercise games may have positive effects for the elderly with respect to perception, memory, and depression. Fallavicini et al. (2020) have carried out a study of young adults $(n=36)$ to explore the ability of VR games to induce positive emotions (e.g., joy and happiness) and reduce negative emotions (e.g. fear and sadness). They found a statistically significant increase in happiness and feelings of surprise; meanwhile, fear and anxiety diminished significantly.

\section{SWOT Analysis on Mental Health of Virtual Reality Games}

In terms of advantages, VR games offer the benefits of VR and the enjoyment of games. Greg et al. (2007) have noted that the potential benefits of VR may vary. Overall, VR games can be an important means of improving the quality of physical and mental health. Students can use VR games to overcome their fears and partake in a range of experiences. In this regard, Singh et al. (2020) have stated that VR improved the skills and confidence of medical workers by providing an advantage in defending against and coping with COVID-19.

With respect to disadvantages, Yildirim (2019) has found that VR games can cause more intense cyber motion sickness compared to regular desktop games. Severe cyber motion sickness symptoms can lead to a lower level of enjoyment. The displays of VR devices may also have a direct negative effect on people. In addition, since VR games are expensive, they may be shared by many people, which introduces hygiene-related concerns (Table 1).

\section{Conclusion/Discussion}

This paper has synthesized data from research on the effects of VR games on mental health in college students. A total of seven studies on VR games and mental health were subject to the final analysis. Overall, the use of VR games among individuals of various ages had positive impacts on the mental health of most participants (e.g., mitigating depression, anxiety, fear). Thus, this paper highlights the potential of VR games to improve mental health.

This paper has conducted a literature review for the purpose of assessing how VR games can influence the mental health of college students. Most of the articles in the review reveal that VR games may improve mental health. Although the results are encouraging, certain limitations should be considered. Specifically, the sample of the study is relatively small, and most of the research was conducted in controlled trials. Thus, it should be reviewed carefully.

Journal of Health, Sports, \& Kinesiology | ISSN 2692-9864 (Online) \& ISSN 2767-5874 (Print)| www.johsk.com 
| 2021 | Volume 2 | Issue 3(S) | The Journal of Health, Sports, and Kinesiology |

Table 1. Strengths, Weakness, Opportunity, and Threats Analysis (SWOT) on Mental Health of Virtual Reality Games.

\begin{tabular}{c|c}
\hline $\begin{array}{c}\text { Strengths (S) } \\
\text { Improve physical \& mental health, } \\
\text { Enjoyment of games }\end{array}$ & $\begin{array}{c}\text { Weaknesses (W) } \\
\text { Cyber motion sickness, } \\
\text { Lead to a lower level of enjoyment }\end{array}$ \\
\hline $\begin{array}{c}\text { Opportunity (O) } \\
\text { Improving the quality of physical and mental health, } \\
\text { Overcome their fears and partake in a range of } \\
\text { experiences }\end{array}$ & $\begin{array}{c}\text { Threats (T) } \\
\text { VR games are expensive, } \\
\text { Hygiene-related concerns }\end{array}$ \\
\hline
\end{tabular}

Through a review of the literature on VR games, this study has illustrated the potential efficacy of VR games for supporting the mental health of college students. However, the data on VR are notably insufficient. Since VR gaming is a new field, there is scarce research on its relation to mental health. In the literature selection process, it was difficult to find data on VR games and mental health, as most of the results related only to VR or video games. According to Srivastava et al. (2014), the technology is still in its infancy, and it requires extensive work, including controlled tests, before it can enter routine use. The definition of VR games is insufficient and in the development stage. One implication of existing studies is that further research should include variables, such as cybersickness, in studies of VR games and health. The research area of VR games is currently limited, and more studies are needed in the future.

\section{References}

Bin Song, G., \& Cho Park, E. (2015). Effect of virtual reality games on stroke patients' balance, gait, depression, and interpersonal relationships. Journal of Physical Therapy Science, 27 (7), 20572060.

Denney, A. S., \& Tewksbury, R. (2013). How to write a literature review. Journal of criminal justice education, 24(2), 218-234.

Gregg, L., \& Tarrier, N. (2007). Virtual reality in mental health. Social Psychiatry and Psychiatric Epidemiology, 42(5), 343-354.

Kyaw, B. M., Saxena, N., Posadzki, P., Vseteckova, J., Nikolaou, C. K., George, P. P., ... \& Car, L. T. (2019). Virtual reality for health professions education: systematic review and meta-analysis by the digital health education collaboration. Journal of Medical Internet Research, 21 (1), el 2959.

Lee, S. Y., Lee, S. K., Kim, Y. H., \& Chae, J. B. (2012). The effects of 4 weeks training using virtual reality game on balance and gait ability, depression of elderly people. PNF and Movement, 10 (2), 41-46.

Pallavicini, F., \& Pepe, A. (2020). Virtual reality games and the role of body involvement in enhancing positive emotions and decreasing anxiety: Within-subjects pilot study. JMIR Serious Games, 8(2), e15635.

Journal of Health, Sports, \& Kinesiology | ISSN 2692-9864 (Online) \& ISSN 2767-5874 (Print) | www.johsk.com 
Rodriguez, A., Rey, B., Vara, M. D., Wrzesien, M., Alcaniz, M., Banos, R. M., \& Perez-Lopez, D. (2015). A VR-based serious game for studying emotional regulation in adolescents. IEEE Computer Graphics and Applications, 35(1), 65-73.

Shaw, A. J., \& Lubetzky, A. V. (2020). A short bout of exercise with and without an immersive virtual reality game can reduce stress and anxiety in adolescents: A pilot randomized controlled trial. Frontiers in Virtual Reality, 1, 43.

Shin, J. H., Park, S. B., \& Jang, S. H. (2015). Effects of game-based virtual reality on health-related quality of life in chronic stroke patients: A randomized, controlled study. Computers in Biology and Medicine, 63, 92-98.

Singh, R. P., Javaid, M., Kataria, R., Tyagi, M., Haleem, A., \& Suman, R. (2020). Significant applications of virtual reality for COVID-19 pandemic. Diabetes \& Metabolic Syndrome. Clinical Research \& Reviews, 14(4), 661-664.

Srivastava, K., Das, R. C., \& Chaudhury, S. (2014). Virtual reality applications in mental health: Challenges and perspectives. Industrial Psychiatry Journal, 23(2), 83.

Yen, H. Y., \& Chiu, H. L. (2021). Virtual reality exergames for improving older adults' cognition and depression: A systematic review and meta-analysis of randomized control trials. Journal of the American Medical Directors Association, 22 (5), 995-1002.

Yildirim, C. (2019). Cybersickness during VR gaming undermines game enjoyment: A mediation model. Displays, 59, 35-43.

\section{Author Information}

Se Ik Park, https://orcid.org/0000-0002-6960-190X

Graduate Student, Department of Instructional Design and Technology, College of Education, California State University, San Bernardino, CA, USA. Email: 006705654@cayote.csusb.edu

Eun-Ok Baek, Ph.D., https://orcid.org/0000-0002-0697-6763

Professor, Department of Instructional Design and Technology, College of Education, California State University, San Bernardino, CA, USA. Email: ebaek@csusb.edu

Hosung So, Ph.D. https://orcid.org/0000-0002-3871-2383

Professor, Department of Kinesiology, College of Natural Sciences, California State University, San Bernardino, CA, USA. Email: hosungso@csusb.edu

\section{Funding Acknowledgement}

Scholarship funding was provided by the IOHSK 2021 Graduate Student Research Grant. This article was written with the support of the IOHSK 2021 Graduate Student Research Grant.

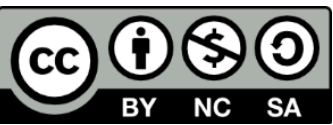

(C) 2021. This work is licensed under a CC BY-NC-SA 4.0 International license.

This is an open access article distributed under the Creative Commons Attribution License which permits unrestricted use, distribution, and reproduction in any medium, provided the original work is properly cited.

Journal of Health, Sports, \& Kinesiology | ISSN 2692-9864 (Online) \& ISSN 2767-5874 (Print) | www.johsk.com 
| 2021 | Volume 2 | Issue 3(S) | The Journal of Health, Sports, and Kinesiology |

Journal of Health, Sports, and Kinesiology, 2021, 2(3), 82-85. https://doi.org/10.47544/johsk.2021.2.3

(c) 2020-2021 by the International Organization for Health, Sports, \& Kinesiology

\section{RESEARCH GRANT REPORT}

\section{Physical Preparation for Junior Tennis Players \\ Alexandru Nicolae Banciu \& Germina Cosma \\ University of Craiova, Romania}

Keywords: junior tennis player, physical preparation, physical tests

\section{Introduction}

The continuous transformation of competitive tennis and physical performances represent the starting point in finding solution to develop the physical preparation. In the last decade, an increase rate of specific injuries was considered the main limitation in achieving high level results. All these conducted to some physical tests implemented for all junior tennis players. For each test a scale was established in order to make a proper evaluation of tennis player that was investigated. The test scale allows the coaches to analyze the player physical performances and to set general training direction in order to improve the player results. All measurement is considered when analyzing a junior tennis player physical performance. By motivating the coaches and players to work and practice better, general and individual adjustments of the training programs are ensured.

To analyze the junior player long term development, initial measurements are mandatory. During the analyze, unique test procedures and complex test are used. All these data are further used to adapt the training and to make it more efficient.

The tennis match can be characterized by intermittent efforts of the whole body because it consists of alternating short attacks (that take between 1 and 9 seconds) with high intensity hits and short recovery (10-19 seconds). Between the games, when they are changing courts, the players have a longer rest period ( 90 seconds). The match duration is usually around 2 hours. During a point a player usually run 8-15 $\mathrm{m}$ with 3-4 changes of direction, hitting the ball 4-5 times (depending on the level of the player and the surface (slow or fast)). The players need to react quickly and to move multidirectional.

Thus, during the tennis match, the demands alternate between providing energy for high intensity attacks / strokes (through intramuscular phosphates and glycolysis) and replenishing energy sources and restoring homeostasis in time intervals (through oxidative metabolism). Therefore, in order to be successful in the competition and to tolerate the demands of intensive training, tennis players need a mixture of speed, agility and power, combined with medium-high aerobic and anaerobic capacities linked to whole body muscle groups.

Journal of Health, Sports, \& Kinesiology | ISSN 2692-9864 (Online) \& ISSN 2767-5874 (Print)| www.johsk.com 
| 2021 | Volume 2 | Issue 3(S) | The Journal of Health, Sports, and Kinesiology |

\section{Purpose}

Test scales are used to examine the abilities of junior players for performances. We used laboratory tests for basic physical performances analyzes and specific tests for a better evaluation of the tennis players. The physical profile of the junior player was made based on the tests results. This profile was used to develop individual training program.

The objective of this research is to describe, evaluate and analyze physical test performances for different players and to use these results for physical training optimization. Specific methods are used in the training program to develop the technical skills of the junior tennis player.

\section{Methods}

Laboratory and specific tests are used to analyze the physical condition of the tennis player. We used laboratory tests to measure the aerobic power. The duration and the intensity of the test conducted to various results. We performed different incrementation steps, different time intervals and number of stages. The results obtained for the male tennis players in our club can be summarized as follow: for the anthropometry test we obtain height $148.9 \mathrm{~cm}$ for U12, $169.4 \mathrm{~cm}$ for U14 and $178.1 \mathrm{~cm}$ for U16, weight $44.8 \mathrm{~kg} \mathrm{U12}, 56.3 \mathrm{~kg} \mathrm{U1} 4$ and $62.6 \mathrm{~kg} \mathrm{U16}$ and Body Mass Index 16.8 U12, 18.5 U14 and 19.9 U16; for the strength and power test we obtained grip strength (dominant hand) $21.3 \mathrm{~kg} \mathrm{U12}, 23.5 \mathrm{~kg}$ U14

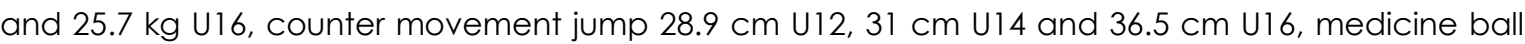
throw 502.6 U12, 612.3 U14 and 713.2 U16.

These laboratory results were obtained that are further used to establish specific tests that are performed by each junior player on the tennis court. The endurance of the player is very important during a tennis match. So, this physical skill should be improved for every junior that perform competitive tennis. In the tennis literature we can find many resistance tests that can be used to evaluate a junior player. To evaluate the endurance of the tennis player we used the Hot and Return test and we obtained the values: level 12.5 for U12, level 14.2 for U14 and level 15.7 for U16.

The service velocity was also measured, and the following results were obtained: $131.5 \mathrm{~km} / \mathrm{h}$ for U12, $157.7 \mathrm{~km} / \mathrm{h}$ for U14 and $170.1 \mathrm{~km} / \mathrm{h}$ for U16.

We also tested the speed and agility of the juniors in our club: for $10 \mathrm{~m}$ we obtained $2.05 \mathrm{~s} \mathrm{U12,1.95 \textrm {s }}$ U14 and 1.84 s U16; for $20 \mathrm{~m}$ we obtained 3.66 s U12, $3.37 \mathrm{~s} \mathrm{U114}$ and $3.22 \mathrm{~s}$ U16; for sprint forehand, we obtained $3.96 \mathrm{~s}$ U12, $2.93 \mathrm{~s}$ U1 4 and $2.73 \mathrm{~s}$ U16 and for sprint backhand we obtained $3.12 \mathrm{~s}$ U12, $3.08 \mathrm{~s}$ U14 and 2.94 s U16.

Main objective of these physical test was to make the junior tennis players aware of the importance of physical training in the process of training for the high-performance. Practical tests of the physical training level are used in order to accumulate concrete and specific data that are further used to develop optimal training programs. All tests' results, grouped by age and sex, are saved in databases and then they are analyzed. The correct quantification of physical training current level is ensured by physical trainers that support and manage the tests. During the analyzation phase, the evolution of each tennis player from one test to another (progress, regression, stagnation) was correlated with the past scale imposed for the player age and sex group. The increase of the values registered from year to year for each tennis player in relation to his physical potential and, at the same time, with the specific requirements of the international performance is a mandatory goal for achieving great

Journal of Health, Sports, \& Kinesiology | ISSN 2692-9864 (Online) \& ISSN 2767-5874 (Print)| www.johsk.com 
performances. The training program will include specific tennis lessons as well as physical training. It is recommended to have a reference physical trainer to deal with the programming and planning of physical training. The coach - physical trainer relationship is very important. The coach is the one that must coordinate the entire training, but he must be in permanent interaction with the physical trainer. Most physical trainers come from other sports, which leads to ignorance of the specifications, methods and means necessary to train a tennis player.

There is still no emphasis on the development of physical capacity gradually and methodically, from an early age, most tennis players starting too late to work physically. However, as a result of the introduction of physical tests, the tested tennis players registered higher values compared to the first indices, which denotes an increase of their interest towards the physical training segment.

\section{Results}

From our study, we can conclude that the level of physical training at the end of the competition season is greater compared to the beginning of the competition season. The tennis players are training very hard through summer competitions and do not have a proper training during the winter. If we make some evaluation tests, we can observe that the values recorded in the spring are often lower than in winter. The short-term objectives (the accumulation of national and international points) often represent real barriers in order to establish a competitive program suitable for each tennis player, the physical training becoming the most often completely ignored or neglected. The test results were used to develop an individual database that was further used to create an effective physical training program. Each individual result wa compared with the category results, and, based on the percentages computed, the coaches and the physical trainers were able to create specific training sessions (based on weakness and strengths) that can conduct to physical performance improvement.

\section{Conclusion/Discussion}

This study was conducted in order to obtain physical improvement of junior tennis players related to their performance level, since the observed differences between performance levels of tennis players remain almost constant from when they pass from U1 4 category to U16 category. Since not all tennis performance can be explained by physical components, other performance parameters such as technique, tactical and psychological elements, need to be included in the evaluation program. The training must conduct to tennis performance improvement by taking in consideration these parameters.

\section{References}

Baiget $\mathrm{E}$, Fernández-Fernández $\mathrm{J}$, Iglesias $\mathrm{X}$, et al. On-court endurance and performance testing in competitive male tennis players. J Strength Cond Res 2014;28:256-64.

Birrer R, Levine R, Gallippi L, et al. The correlation of performance variables in preadolescent tennis players. J Sports Med Phys Fitness 1986;26:137.

Buchheit M. The 30-15 intermittent fitness test: accuracy for individualizing interval training of young intermittent sport players. J Strength Cond Res 2008;22:365-74.

Fernandez J, Mendez-Villanueva A, Pluim B. Intensity of tennis match play. Br J Sports Med 2006:40:387-91.

Journal of Health, Sports, \& Kinesiology | ISSN 2692-9864 (Online) \& ISSN 2767-5874 (Print) | www.johsk.com 
Fernandez J, Sanz-Rivas D, Mendez-Villanueva A. A review of the activity profile and physiological demands of tennis match play. Strength Cond J 2009:31:15-26.

Ferrauti A, Weber K, Wright P. Endurance: basic, semi-specific and specific. Strength and conditioning for tennis. London: ITF, 2003:93-111.

Girard O, Millet GP. Physical determinants of tennis performance in competitive teenage players. J Strength Cond Res 2009;23:1867-72.

Kovacs MS. Tennis physiology: training the competitive athlete. Sports Med, 2007;37:189-98.

Reid M, Quinn A, Crespo M. Strength and conditioning for tennis. International Tennis Federation, 2003.

Roetert E, Piorkowski P, Woods R, et al. Establishing percentiles for junior tennis players based on physical fitness testing results. Clin Sports Med 1995;14:1.

Roetert P, Ellenbecker TS. Complete conditioning for tennis. Human Kinetics Publishers, 2007.

Smekal G, Pokan R, von Duvillard SP, et al. Comparison of laboratory and 'on-court' endurance testing in tennis. Int J Sports Med 2000;21: 242-9.

Ulbricht A, Fernandez-Fernandez J, Ferrauti A. Conception for fitness testing and individualized training programs in the German Tennis Federation. Sports Orthopaedics and Traumatology 2013;29:180-92.

Urso R, Okuno N, Gomes R, et al. Validity and reliability evidences of the Hit \& Turn Tennis Test. Sci Sports 2013.

Vergauwen L, Spaepen AJ, Lefevre J, et al. Evaluation of stroke performance in tennis. Med Sci Sports Exerc 1998;30:1281-8.

\section{Author Information}

Alexandru Nicolae Banciu, https://orcid.org/0000-0003-3447-4637

Doctoral Student, Faculty of Physical Education and Sport, University of Craiova, Romania.

Email: alexandru.banciu@edu.ucv.ro

Germina Cosma, Ph.D., https://orcid.org/0000-0002-4636-8041

Faculty of Physical Education and Sport, University of Craiova, Romania.

Email: germina.cosma@edu.ucv.ro

\section{Funding Acknowledgement}

Scholarship funding was provided by the IOHSK 2021 Graduate Student Research Grant. This article was written with the support of the IOHSK 2021 Graduate Student Research Grant.

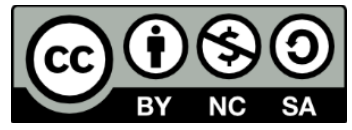

(C) 2021. This work is licensed under a CC BY-NC-SA 4.0 International license.

This is an open access article distributed under the Creative Commons Attribution License which permits unrestricted use, distribution, and reproduction in any medium, provided the original work is properly cited.

Journal of Health, Sports, \& Kinesiology | ISSN 2692-9864 (Online) \& ISSN 2767-5874 (Print) | www.johsk.com 


\title{
34 RESEARCH GRANT PROGRESS REPORT
}

\author{
The U.S. Insulin Crisis \\ Viktoria Layton, Priya Desai, \& Joon Young Kim \\ Syracuse University, New York, USA
}

Keywords: insulin therapy, pharmacology, type 2 diabetes

\section{Progress Report}

Since its century-old discovery, the cost of insulin has substantially increased. Three pharmaceutical companies - Eli Lilly, Novo Nordisk, and Sanofi-Aventis - have sustained a "monopolistic hold" on the insulin market. One 10-ml vial of Humalog, for example, a popular rapid-acting insulin lispro injection manufactured by Eli Lilly, underwent a 685\% price increase from $\$ 35$ in 2001 to $\$ 275$ today. The lack of laws capping price increases coupled with limited competition of biosimilar drugs from entering the market have placed an economic burden on Americans who need insulin to treat their diabetes. Americans who are unable to afford the high costs of insulin have turned to insulin rationing, whether that be skipping drug doses, delaying filling prescriptions, or taking less of the drug than prescribed. Insulin rationing can result in worsened glycemic control and lead to a condition known as diabetic ketoacidosis. The consequences of diabetic ketoacidosis are severe and often fatal. The federal government has recently engaged in insulin price reform. The Affordable Drug Manufacturing Act and the Emergency Access to Insulin Act were introduced to eliminate barriers that exist for competition to enter the market and expand access to insulin supplies to those in significant need; however, the bills have yet to be enacted. States have also attempted to address the problem of insulin affordability. Colorado and Illinois are currently the only states to have passed law legislations that cap out-ofpocket costs of insulin for all diabetics with six other states not far behind. For many, insulin is key to their survival and its affordability should be a concern for all stakeholders involved.

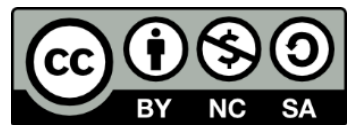

(C) 2021. This work is licensed under a CC BY-NC-SA 4.0 International license.

This is an open access article distributed under the Creative Commons Attribution License which permits unrestricted use, distribution, and reproduction in any medium, provided the original work is properly cited.

\section{Author Information}

Vikłoria Layton, https://orcid.org/0000-0002-2516-9449

Department of Exercise Science, David B. Falk College of Sport and Human Dynamics, Syracuse University, Syracuse, NY, USA. Email: vmlayton@syr.edu

Journal of Health, Sports, \& Kinesiology | ISSN 2692-9864 (Online) \& ISSN 2767-5874 (Print) | www.johsk.com 
| 2021 | Volume 2 | Issue 3(S) | The Journal of Health, Sports, and Kinesiology |

Priya Desai, https://orcid.org/0000-0000-0000-0000

Department of Exercise Science, David B. Falk College of Sport and Human Dynamics, Syracuse University, Syracuse, NY, USA. Email: syu125@syr.edu

Joon Young Kim, Ph.D., https://orcid.org/0000-0003-0448-1684

Assistant Professor, Department of Exercise Science, David B. Falk College of Sport and Human Dynamics, Syracuse University, Syracuse, NY, USA.

Email: jkim291@syr.edu

\section{Funding Acknowledgement}

Scholarship funding was provided by the IOHSK 2021 Graduate Student Research Grant. 
| 2021 | Volume 2 | Issue 3(S) | The Journal of Health, Sports, and Kinesiology |

Journal of Health, Sports, and Kinesiology, 2021, 2(3), 88-89. https://doi.org/10.47544/johsk.202 1.2.3

(c) 2020-2021 by the International Organization for Health, Sports, \& Kinesiology

\title{
35 ONGOING RESEARCH REPORT
}

\section{The Epidemiology of Well-being, Happiness, Perceived Stress, and Health Behaviors of Asian University Students: A Multinational Project}

\author{
Raul Calderon Jr. \\ Former Visiting Professor, Faculty of Sports Science, Kasetsart University, Thailand
}

Keywords: psychological well-being, happiness, stress, health behaviors, students

\begin{abstract}
Introduction: The field of Positive Psychology is broad. One area of special focus is psychological wellbeing (PWB). Research on PWB and its relation to health outcomes has increased over the last 3 decades. However, much of the early research primarily focused on PWB and health outcomes in adults from western societies. There remains a lack of research using a theory-based operationalized definition for PWB and the use of a broader spectrum of health risk factors in student populations. To advance the field of PWB and student health, it is recommended that current theory-based constructs of PWB and a comprehensive list of health factors be utilized when investigating associations between PWB and health outcomes in Asian university students.
\end{abstract}

Purpose: This project aimed to (1) create a comprehensive questionnaire to assess PWB, happiness, health risk factors, and stress, (2) recruit universities from different Asian countries to administer the questionnaire to selected students, (3) validate the questionnaire for each country, (4) analyze data at the country and cross-cultural levels, and (5) present, publish, and disseminate finding.

Methods: Researchers from India, Thailand, and Vietnam were recruited to develop, translate, validate, and pilot a final research questionnaire consisting of the following constructs/instruments: (1) PWB/Ryff Scales of Psychological Well-being, (2) Happiness/Subjective Happiness Scale, (3) Mindfulness/Mindfulness Attention Awareness Scale, (4) Stress/Perceived Stress Scale, (5) Mental Health/depressive symptoms, (6) Religiosity/regular practice, (7) Health Literacy/courses and knowledge, (8) Risk Assessment/Perceived health risk, (9) Health Behaviors/alcohol, diet, sleep, and smoking, and (10) Demographic information/standardized questions. The final questionnaire was administered to undergraduate students (including $1^{\text {st }}, 2^{\text {nd }}, 3^{\text {rd }}$, and $4^{\text {th }}$ year) from India (two universities, $N=508$ ), Thailand (two universities, $N=1132$ ), and Vietnam (three universities, $N=1821$ ). Students completed an online or paper survey in a classroom setting from October 2016-December 2017. The questionnaire consisted of 115 questions and was completed in an average of 15-20 minutes. The main component to assess PWB included the 54-item Ryff scales of psychological well-being (RSPWB), consisting of six subscales (autonomy, environmental mastery, purpose in life, positive relations, personal growth, and self-acceptance), with 9-items per scale, and contained a six-point Likert scale

Journal of Health, Sports, \& Kinesiology | ISSN 2692-9864 (Online) \& ISSN 2767-5874 (Print) | www.johsk.com 
(from 1=Strongly Disagree to $6=$ Strongly Agree). A composite PWB score (sum of all subscales) was calculated for each country. Data were analyzed using univariate, bivariate, ANOVA, Post Hoc, regression, and structural equation modeling statistics on SPSS (v2019) and SmartPLSV2.

Results: Multiple papers have been published in peer-review journals over the last 3 years. The first set of papers are focused on country level analyses. The cross-cultural analyses will follow shortly.

\section{Vietnam}

Thi, N. N., Calderon Jr, R., Quang, T. T., Trung, K. N., Chi, Q., Thai, N., Thanh, T. H., Minh, T. N. H., Viet, V., \& Thanh, H. N. (2020). Happiness, stress, depressive symptoms, and health behaviors among Vietnamese university students. Journal of Public Health and Development, 18(2), 58-73.

Calderon Jr, R., Nga, N. T., Tien, T. Q., Quyen, B. T. T., Thuan, N. H. M., \& Bao, V. V. (2019). Adapting the Ryff scales of psychological well-being: A 28-Item Vietnamese version for university students. VNU Journal of Social Sciences and Humanities, 5(2), 176-197.

\section{Thailand}

Calderon, R., Pupanead, S., Prachakul, W., \& Kim, G. (2021). Happiness, perceived stress, psychological well-being, and health behaviors of Thai university students: Preliminary results from a multinational study on well-being. Journal of American College Health, 69(2), 176-184.

https://doi.org/10.1080/07448481.2019.1657871

Calderon Jr, R., Kim, G., Ratsameemonthon, C., \& Pupanead, S. (2020). Assessing the Adaptation of a Thai Version of the Ryff scales of psychological well-being: A PLS-SEM approach. Psychology, 11 (7), 1037-1053. https://doi.org/10.4236/psych.2020.117068

India: Papers in progress

Cross-cultural papers: A Cross-sectional, Cross-cultural, Comparative Study: A Drill-down analysis of psychological well-being among Asian students from India, Thailand, and Vietnam (Oral presentation at IOHSK Online Conference, October 7-9, 2021)

Discussion: This project has been successful, thus far, in engaging researchers from multiple countries to contribute needed data in the area of Asian student health. A balanced questionnaire was developed, piloted, translated, and administered at universities in 3 Asian countries. Multiple papers validating the questionnaire and describing associations between PWB and health factors have been published in peer-reviewed scientific journals. Additional research questions will be formulated and explored in the large dataset created by this project. We expect to present and publish additional papers concerning PWB and Asian university student health over the next few years.

\section{Author Information}

Raul Calderon Jr. Ph.D., https://orcid.org/0000-0002-7580-3057

Former Visiting Professor, Faculty of Sports Science, Kasetsart University, Thailand \& Executive Advisor, International Organization of Health, Sports, and Kinesiology (IOHSK), USA.

Email: raulthedoc@gmail.com

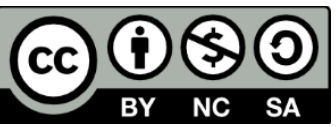

(c) 2021. This work is licensed under a CC BY-NC-SA 4.0 International license.

This is an open access article distributed under the Creative Commons Attribution License which permits unrestricted use, distribution, and reproduction in any medium, provided the original work is properly cited.

Journal of Health, Sports, \& Kinesiology | ISSN 2692-9864 (Online) \& ISSN 2767-5874 (Print) | www.johsk.com 


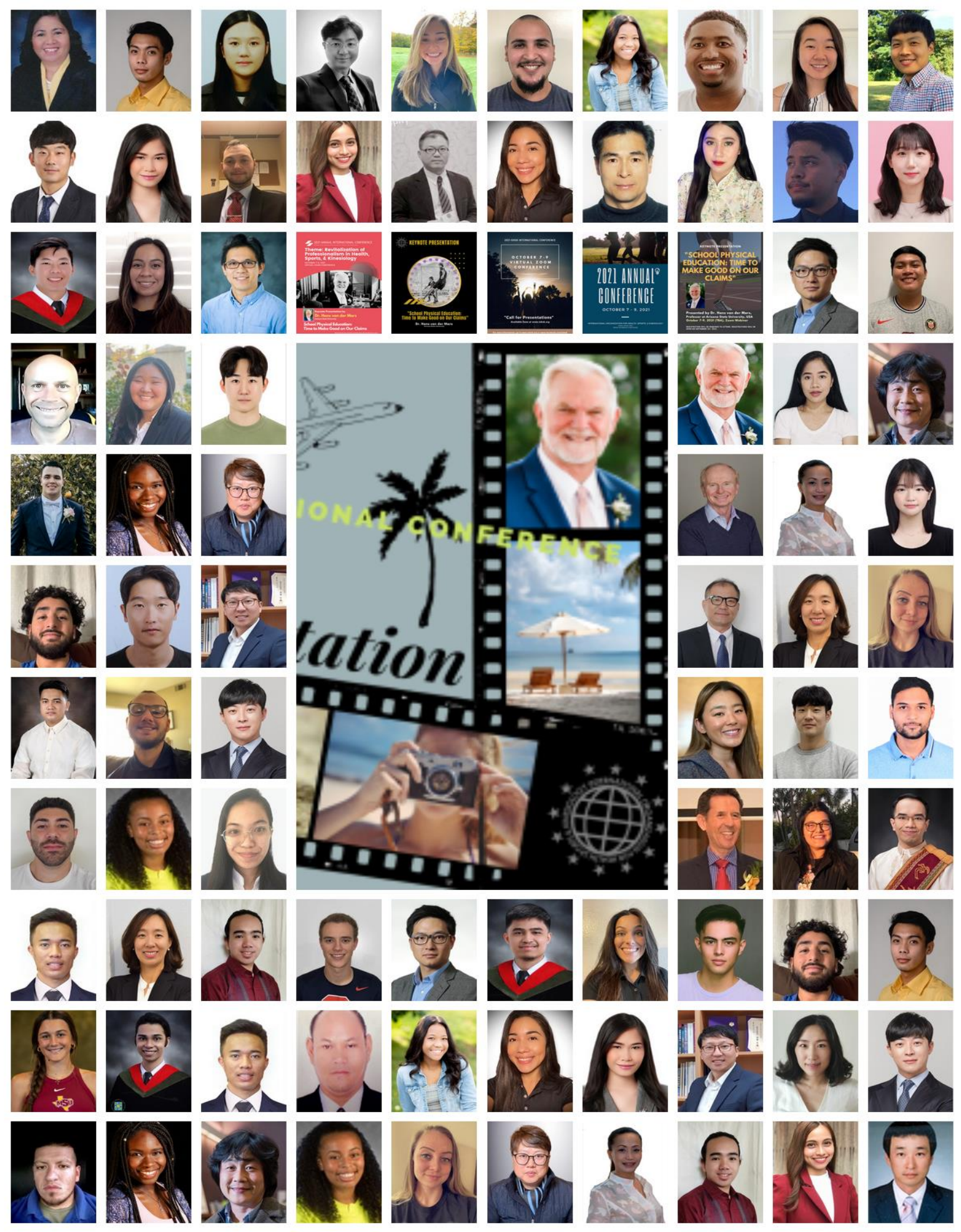




\section{IOHSK INTERNATIONAL CONFERENCE}

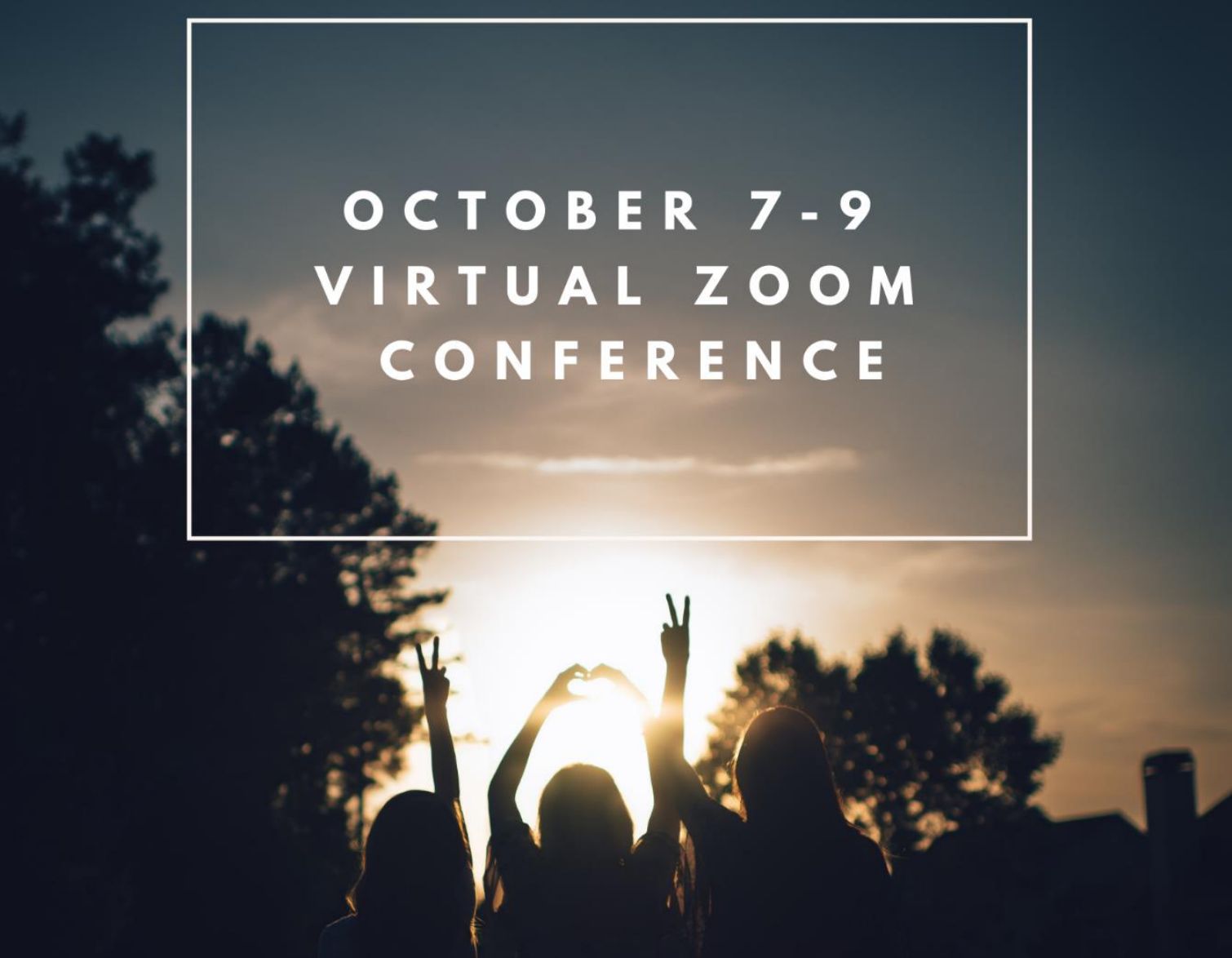

\section{"Call for Presentations"}

Available Soon at www.iohsk.org

For more information, go to www.iohsk.org or www.facebook.com/iohsk 


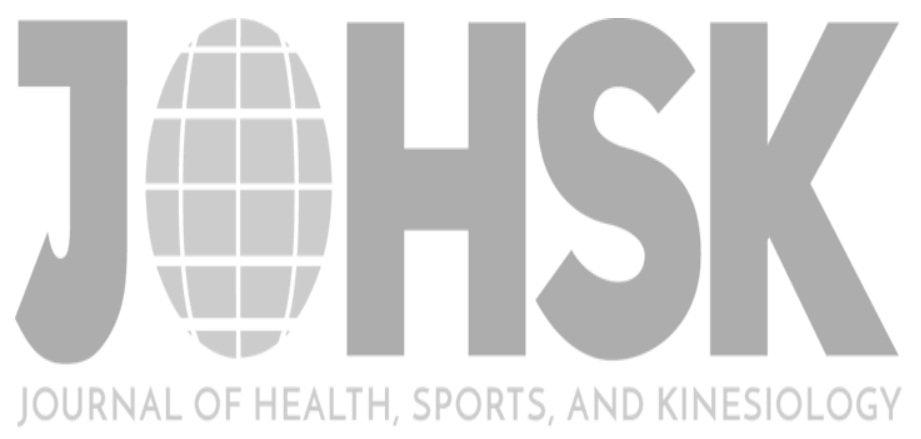

JOHSK ISSN

2692-9864 (Online) \& 2767-5874 (Print)

Volume URL

https://www.johsk.com/2021-vol2-issue3

Volume DOI

https://doi.org/10.47544/johsk.2021.2.3.0192

Issue DOI

https://doi.org/10.47544/johsk.2021.10.22

Article DOI

https://doi.org/10.47544/johsk.2021.2.3
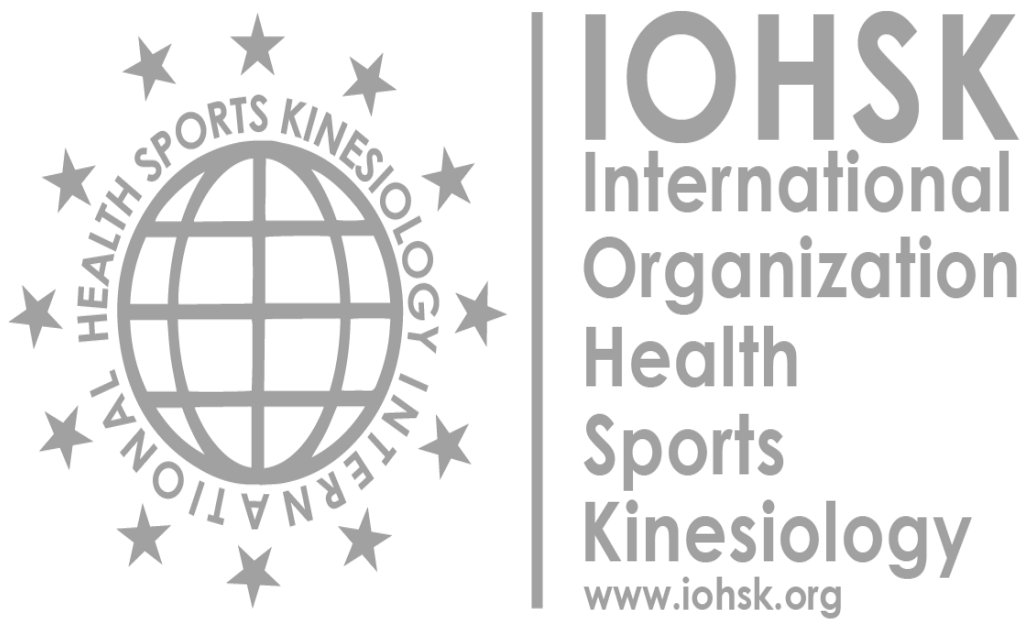

Health

Sports

Kinesiology

www.iohsk.org 